\title{
GUIDELINES
}

\section{Surviving sepsis campaign: international guidelines for management of sepsis and septic shock 2021}

\author{
Laura Evans ${ }^{1 *} \mathbb{D}$, Andrew Rhodes ${ }^{2}$, Waleed Alhazzani ${ }^{3}$, Massimo Antonelli ${ }^{4}$, Craig M. Coopersmith ${ }^{5}$, \\ Craig French ${ }^{6}$, Flávia R. Machado ${ }^{7}$, Lauralyn Mcintyre ${ }^{8}$, Marlies Ostermann ${ }^{9}$, Hallie C. Prescott ${ }^{10}$, \\ Christa Schorr ${ }^{11}$, Steven Simpson ${ }^{12}$, W. Joost Wiersinga ${ }^{13}$, Fayez Alshamsi ${ }^{14}$, Derek C. Angus ${ }^{15}$, Yaseen Arabi ${ }^{16}$, \\ Luciano Azevedo ${ }^{17}$, Richard Beale ${ }^{9}$, Gregory Beilman ${ }^{18}$, Emilie Belley-Cote ${ }^{19}$, Lisa Burry ${ }^{20}$, Maurizio Cecconi ${ }^{21,22}$, \\ John Centofanti ${ }^{23}$, Angel Coz Yataco ${ }^{24}$, Jan De Waele ${ }^{25}$, R. Phillip Dellinger ${ }^{11}$, Kent Doi ${ }^{26}$, Bin Du ${ }^{27}$, \\ Elisa Estenssoro ${ }^{28}$, Ricard Ferrer ${ }^{29}$, Charles Gomersall ${ }^{30}$, Carol Hodgson ${ }^{31}$, Morten Hylander Møller ${ }^{32}$, \\ Theodore Iwashyna ${ }^{33}$, Shevin Jacob ${ }^{34}$, Ruth Kleinpel ${ }^{35}$, Michael Klompas ${ }^{36,37}$, Younsuck Koh ${ }^{38}$, Anand Kumar ${ }^{39}$, \\ Arthur Kwizera ${ }^{40}$, Suzana Lobo ${ }^{41}$, Henry Masur ${ }^{42}$, Steven McGloughlin ${ }^{43}$, Sangeeta Mehta ${ }^{44}$, Yatin Mehta ${ }^{45}$, \\ Mervyn Mer ${ }^{46}$, Mark Nunnally ${ }^{47}$, Simon Oczkowski ${ }^{3}$, Tiffany Osborn ${ }^{48}$, Elizabeth Papathanassoglou ${ }^{49}$, \\ Anders Perner ${ }^{50}$, Michael Puskarich ${ }^{51}$, Jason Roberts ${ }^{52,53,54,55}$, William Schweickert ${ }^{56}$, Maureen Seckel ${ }^{57}$, \\ Jonathan Sevransky ${ }^{5}$, Charles L. Sprung ${ }^{58,59}$, Tobias Welte ${ }^{60}$, Janice Zimmerman ${ }^{61}$ and Mitchell Levy ${ }^{62}$
}

Keywords: Sepsis, Septic shock, Adults, Guidelines, Evidence based medicine

\section{Introduction}

Sepsis is life-threatening organ dysfunction caused by a dysregulated host response to infection [1]. Sepsis and septic shock are major healthcare problems, impacting millions of people around the world each year and killing between one in three and one in six of those it affects [2-4]. ${ }^{1}$ Early identification and appropriate management in the initial hours after the development of sepsis improve outcomes.

The recommendations in this document are intended to provide guidance for the clinician caring for adult patients with sepsis or septic shock in the hospital setting. Recommendations from these guidelines cannot

\footnotetext{
*Correspondence: leevans@uw.edu

${ }^{1}$ Division of Pulmonary, Critical Care and Sleep Medicine, University of Washington, Seattle, WA, USA

Full author information is available at the end of the article
}

This article is co-published in the journals Intensive Care Medicine (https://doi.org/10.1007/s00134-021-06506-y) and Critical Care Medicine (https://doi.org/10.1097/CCM.0000000000005337). All Rights Reserved.

\section{Springer}

replace the clinician's decision-making capability when presented with a unique patient's clinical variables. These guidelines are intended to reflect best practice (Table 1).

\section{Screening and early treatment}

Screening for patients with sepsis and septic shock

\section{Recommendation}

1. For hospitals and health systems, we recommend using a performance improvement programme for sepsis, including sepsis screening for acutely ill, high-risk patients and standard operating procedures for treatment

Strong recommendation, moderate quality of evidence for screening

Strong recommendation, very low-quality evidence for standard operating procedures

\section{Rationale}

Sepsis performance improvement programmes generally consist of sepsis screening, education, measurement

${ }^{1}$ References 5-24 are referred to in the Electronic Supplementary Material "Methodology" that can be accessed online at https://doi.org/10.1007/ s00134-021-06506-y. 
of sepsis bundle performance, patient outcomes, and actions for identified opportunities [25, 26]. Despite some inconsistency, a meta-analysis of 50 observational studies on the effect of performance improvement programmes showed that these programmes were associated with better adherence to sepsis bundles along with a reduction in mortality (OR 0.66 ; 95\% CI $0.61-0.72$ ) in patients with sepsis and septic shock [27]. The specific components of performance improvement did not appear to be as important as the presence of a programme that included sepsis screening and metrics.

Sepsis screening tools are designed to promote early identification of sepsis and consist of manual methods or automated use of the electronic health record (EHR). There is wide variation in diagnostic accuracy of these tools with most having poor predictive values, although the use of some was associated with improvements in care processes [28-31]. A variety of clinical variables and tools are used for sepsis screening, such as systemic inflammatory response syndrome (SIRS) criteria, vital signs, signs of infection, quick Sequential Organ Failure Score (qSOFA) or Sequential Organ Failure Assessment (SOFA) criteria, National Early Warning Score (NEWS), or Modified Early Warning Score (MEWS) [26, 32]. Machine learning may improve performance of screening tools, and in a meta-analysis of 42,623 patients from seven studies for predicting hospital-acquired sepsis the pooled area under the receiving-operating curve (SAUROC) $(0.89$; 95\% CI 0.86-0.92); sensitivity $(81 \%$; $95 \%$ CI $80-81$ ), and specificity (72\%; 95\% CI 72-72) was higher for machine learning than the SAUROC for traditional screening tools such as SIRS (0.70), MEWS (0.50), and SOFA (0.78) [32].

Screening tools may target patients in various locations, such as in-patient wards, emergency departments, or intensive care units (ICU) [28-30, 32]. A pooled analysis of three RCTs did not demonstrate a mortality benefit of active screening (RR 0.90; 95\% CI 0.51-1.58) [33-35]. However, while there is wide variation in sensitivity and specificity of sepsis screening tools, they are an important component of identifying sepsis early for timely intervention.

Standard operating procedures are a set of practices that specify a preferred response to specific clinical circumstances [36]. Sepsis standard operating procedures, initially specified as Early Goal Directed Therapy have evolved to "usual care" which includes a standard approach with components of the sepsis bundle, early identification, lactate, cultures, antibiotics, and fluids [37]. A large study examined the association between implementation of state-mandated sepsis protocols, compliance, and mortality. A retrospective cohort study of 1,012,410 sepsis admissions to 509 hospitals in the United States in a retrospective cohort examined mortality before (27 months) and after (30 months) implementation of New York state sepsis regulations, with a concurrent control population from 4 other states [38]. In this comparative interrupted time series, mortality was lower in hospitals with higher compliance with achieving the sepsis bundles successfully.

Lower resource countries may experience a different effect. A meta-analysis of 2 RCTs in Sub-Saharan Africa found higher mortality (RR 1.26; 95\% CI 1.00-1.58) with standard operating procedures compared with usual care, while it was decreased in one observational study (adjusted hazard ratio [HR]; 95\% CI 0.55-0.98) [39].

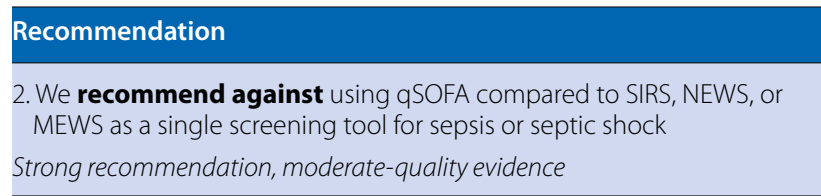

\section{Rationale}

The qSOFA uses 3 variables to predict death and prolonged ICU stay in patients with known or suspected sepsis: a Glasgow Coma Score $<15$, a respiratory rate $\geq 22$ breaths/min and a systolic blood pressure $\leq 100 \mathrm{mmHg}$. When any two of these variables are present simultaneously the patient is considered to be qSOFA positive. Data analysis used to support the recommendations of the 3rd International Consensus Conference on the Definitions of Sepsis identified qSOFA as a predictor of poor outcome in patients with known or suspected infection, but no analysis was performed to support its use as a screening tool [5]. Since that time numerous studies have investigated the potential use of the qSOFA as a screening tool for sepsis [40-42]. The results have been contradictory as to its usefulness. Studies have shown that qSOFA is more specific but less sensitive than having two of four SIRS criteria for early identification of infection induced organ dysfunction [40-43]. Neither SIRS nor qSOFA are ideal screening tools for sepsis and the bedside clinician needs to understand the limitations of each. In the original derivation study, authors found that only $24 \%$ of infected patients had a qSOFA score 2 or 3 , 
but these patients accounted for $70 \%$ of poor outcomes [5]. Similar findings have also been found when comparing against the National Early warning Score (NEWS) and the Modified Early warning Score (MEWS) [44]. Although the presence of a positive qSOFA should alert the clinician to the possibility of sepsis in all resource settings; given the poor sensitivity of the qSOFA, the panel issued a strong recommendation against its use as a single screening tool.

Recommendation
3. For adults suspected of having sepsis, we suggest measuring blood
lactate
Weak recommendation, low-quality evidence

\section{Rationale}

The association of lactate level with mortality in patients with suspected infection and sepsis is well established $[45,46]$. Its use is currently recommended as part of the SSC Hour-1 sepsis bundle for those patients with sepsis $[47,48]$, and an elevated lactate is part of the Sepsis-3 definition of septic shock [49]. It has been suggested that lactate can also be used to screen for the presence of sepsis among undifferentiated adult patients with clinically suspected (but not confirmed) sepsis. Several studies have assessed the use of lactate in this context [50-52].

The lactate cutoffs determining an elevated level ranged from 1.6 to $2.5 \mathrm{mmol} / \mathrm{L}$, although diagnostic characteristics were similar regardless of the cutoff. Sensitivities range from 66 to $83 \%$, with specificities ranging from 80 to $85 \%$. Pooled positive and negative likelihood ratios from the three studies are 4.75 and 0.29 , respectively. Studies showed an association between the use of point-of-care lactate measurements at presentation and reduced mortality; however, the results are inconsistent [53]. In summary, the presence of an elevated or normal lactate level significantly increases or decreases, respectively, the likelihood of a final diagnosis of sepsis in patients with suspected sepsis. However, lactate alone is neither sensitive nor specific enough to rule-in or rule-out the diagnosis on its own. Lactate testing may not be readily available in many resource-limited settings [54-61]. Therefore, we issued a weak recommendation favouring the use of serum lactate as an adjunctive test to modify the pretest probability of sepsis in patients with suspected but not confirmed sepsis.

\section{Initial resuscitation}

\section{Recommendations}

4. Sepsis and septic shock are medical emergencies, and we recommend that treatment and resuscitation begin immediately

Best Practice Statement

5. For patients with sepsis induced hypoperfusion or septic shock we suggest that at least $30 \mathrm{~mL} / \mathrm{kg}$ of intravenous (IV) crystalloid fluid

should be given within the first $3 \mathrm{~h}$ of resuscitation

Weak recommendation, low-quality evidence

6. For adults with sepsis or septic shock, we suggest using dynamic measures to guide fluid resuscitation, over physical examination or static parameters alone

Weak recommendation, very low-quality evidence

\section{Remarks}

Dynamic parameters include response to a passive leg raise or a fluid bolus, using stroke volume (SV), stroke volume variation (SVV), pulse pressure variation (PPV), or echocardiography, where available

7. For adults with sepsis or septic shock, we suggest guiding resuscitation to decrease serum lactate in patients with elevated lactate level, over not using serum lactate

Weak recommendation, low-quality evidence

Remarks

During acute resuscitation, serum lactate level should be interpreted considering the clinical context and other causes of elevated lactate

8. For adults with septic shock, we suggest using capillary refill time to guide resuscitation as an adjunct to other measures of perfusion Weak recommendation, low-quality evidence

\section{Rationale}

Timely, effective fluid resuscitation is crucial for the stabilisation of sepsis-induced tissue hypoperfusion in sepsis and septic shock. Previous guidelines recommend initiating appropriate resuscitation immediately upon recognition of sepsis or septic shock and having a low threshold for commencing it in those patients where sepsis is not proven but is suspected. Although the evidence stems from observational studies, this recommendation is considered a best practice and there are no new data suggesting that a change is needed.

The 2016 SSC guideline issued a recommendation for using a minimum of $30 \mathrm{ml} / \mathrm{kg}$ (ideal body weight) of IV crystalloids in initial fluid resuscitation. This fixed volume of initial resuscitation was based on observational evidence [62]. There are no prospective intervention studies comparing different volumes for initial resuscitation in sepsis or septic shock. A retrospective analysis of adults presenting to an emergency department with sepsis or septic shock showed that failure to receive $30 \mathrm{ml} / \mathrm{kg}$ of crystalloid fluid therapy within $3 \mathrm{~h}$ of sepsis onset was associated with increased odds of in-hospital mortality, delayed resolution of hypotension and increased length of stay in ICU, irrespective of comorbidities, including end-stage kidney disease and heart failure [63]. In the 
PROCESS [64], ARISE [65] and PROMISE [66] trials, the average volume of fluid received pre-randomisation was also in the range of $30 \mathrm{ml} / \mathrm{kg}$, suggesting that this fluid volume has been adopted in routine clinical practice [67].

Most patients require continued fluid administration following initial resuscitation. Such administration needs to be balanced with the risk of fluid accumulation and potential harm associated with fluid overload, in particular, prolonged ventilation, progression of acute kidney injury (AKI) and increased mortality. One of the most important principles of managing complex septic patients is the need for a detailed initial assessment and ongoing re-evaluation of the response to treatment. To avoid over- and under-resuscitation, fluid administration beyond the initial resuscitation should be guided by careful assessment of intravascular volume status and organ perfusion. Heart rate, central venous pressure (CVP) and systolic blood pressure alone are poor indicators of fluid status. Dynamic measures have demonstrated better diagnostic accuracy at predicting fluid responsiveness compared with static techniques. Dynamic measures include passive leg raising combined with cardiac output $(\mathrm{CO})$ measurement, fluid challenges against stroke volume (SV), systolic pressure or pulse pressure, and increases of SV in response to changes in intrathoracic pressure. In a systematic review and meta-analysis, dynamic assessment to guide fluid therapy was associated with reduced mortality (RR 0.59; 95\% CI 0.42-0.83), ICU length of stay (MD -1.16 days; $95 \% \mathrm{CI}-1.97$ to -0.36 ), and duration of mechanical ventilation $(-2.98 \mathrm{~h} ; 95 \%$ CI -5.08 to -0.89 ) [3]. However, in one other metaanalysis, there was no significant difference in mortality between septic patients resuscitated with a volume responsiveness-guided approach compared with standard resuscitative strategies [68]. Most data arise from high income settings and a paucity of evidence exists in resource-limited settings to guide optimal titration of fluid resuscitation as well as the appropriate safety endpoints. An RCT in patients with sepsis and hypotension in Zambia showed that early protocolised resuscitation with administration of IV fluids guided by jugular venous pressure, respiratory rate, and arterial oxygen saturation only, was associated with significantly more fluid administration in the first $6 \mathrm{~h}$ [median 3.5L (IQR 2.7-4.0) versus 2.0L (IQR 1.0-2.5)] and higher hospital mortality (48.1\% versus $33 \%$ ) than standard care [69].

If fluid therapy beyond the initial $30 \mathrm{ml} / \mathrm{kg}$ administration is required, clinicians may use repeated small boluses guided by objective measures of $\mathrm{SV}$ and/or $\mathrm{CO}$. In post-cardiac surgery patients, fluid challenges of $4 \mathrm{ml} / \mathrm{kg}$ compared to $1-3 \mathrm{ml} / \mathrm{kg}$ increased the sensitivity of detecting fluid responders and non-responders based on measurement of $\mathrm{CO}$ [70]. In resource-limited regions where measurement of $\mathrm{CO}$ or SV may not be possible, a $>15 \%$ increase in pulse pressure could indicate that the patient is fluid responsive utilizing a passive leg-raise test for $60-90$ seconds $[71,72]$.

Serum lactate is an important biomarker of tissue hypoxia and dysfunction, but is not a direct measure of tissue perfusion [73]. Recent definitions of septic shock include increases in lactate as evidence of cellular stress to accompany refractory hypotension [1]. Previous iterations of these guidelines have suggested using lactate levels as a target of resuscitation in the early phases of sepsis and septic shock, based on earlier studies related to goal-directed therapy and meta-analyses of multiple studies targeting reductions in serum lactate in comparison to "standard care" or increases in central venous oxygen saturation $[74,75]$. The panel recognises that normal serum lactate levels are not achievable in all patients with septic shock, but these studies support resuscitative strategies that decrease lactate toward normal. Serum lactate level should be interpreted considering the clinical context and other causes of elevated lactate. As with sepsis screening, lactate measurement may not always be available in some resource-limited settings.

When advanced haemodynamic monitoring is not available, alternative measures of organ perfusion may be used to evaluate the effectiveness and safety of volume administration. Temperature of the extremities, skin mottling and capillary refill time (CRT) have been validated and shown to be reproducible signs of tissue perfusion $[76,77]$. The ANDROMEDA-SHOCK study evaluated whether a resuscitation strategy targeting CRT normalisation was more effective than a resuscitation strategy aiming at normalisation or decreasing lactate levels by $20 \%$ every $2 \mathrm{~h}$ in the first $8 \mathrm{~h}$ of septic shock [58]. At day 3, the CRT group had significantly less organ dysfunction as assessed by SOFA score [mean SOFA score 5.6 (SD 4.3) versus 6.6 (SD 4.7); $p=0.045$ ]. 28-day mortality was $34.9 \%$ in the peripheral perfusion group and $43.4 \%$ in the lactate group, but this difference did not reach statistical significance (HR 0.75; 95\% CI 0.55-1.02). Despite the absence of a clear effect on mortality, using CRT during resuscitation has physiologic plausibility and is easily performed, non-invasive, and no cost. However, this approach should be augmented by careful, frequent, and comprehensive patient evaluation to predict or recognise fluid overload early, particularly where critical care resources are constrained. Relevant consideration of the background pathology or pathological processes pertinent to the patient should also inform management $[69,78]$. 


\section{Mean arterial pressure}

Recommendation

9. For adults with septic shock on vasopressors, we recommend an initia target mean arterial pressure (MAP) of $65 \mathrm{~mm} \mathrm{Hg}$ over higher MAP targets

Strong recommendation, moderate-quality evidence

\section{Rationale}

MAP is a key determinant of mean systemic filling pressure, which in turn, is the major driver of venous return and CO. Increasing MAP therefore usually results in increased tissue blood flow and augments the supply side of tissue perfusion. While some tissues, such as the brain and kidneys have the ability to auto-regulate blood flow, MAPs below a threshold, usually understood to be approximately $60 \mathrm{~mm} \mathrm{Hg}$, are associated with decreased organ perfusion, which tracks linearly with MAP [79]. Previous SSC guidelines recommended targeting a MAP of greater than $65 \mathrm{~mm} \mathrm{Hg}$ for initial resuscitation. The recommendation was based principally on a RCT in septic shock comparing patients who were given vasopressors to target a MAP of $65-70 \mathrm{~mm} \mathrm{Hg}$, versus a target of $80-85 \mathrm{~mm} \mathrm{Hg}$ [80]. This study found no difference in mortality, although a sub-group analysis demonstrated a $10.5 \%$ absolute reduction in renal replacement therapy (RRT) with higher MAP targets among patients with chronic hypertension. In addition, targeting higher MAP with vasopressors was associated with a higher risk of atrial fibrillation. A limitation of this study was that the average MAP in both arms exceeded the targeted range. A meta-analysis of two RCTs on this topic supported that higher MAP targets did not improve survival in septic shock (RR 1.05; 95\% CI 0.90-1.23) [81].

A recent RCT, monitored to ensure protocol and MAP target compliance, compared a "permissive hypotension" (MAP 60-65 mm Hg) group with a "usual care" group that received vasopressors and MAP targets set by the treating physician in patients aged 65 years and older with septic shock $[82,83]$. The intervention group in this study achieved a mean MAP of $66.7 \mathrm{~mm} \mathrm{Hg}$, compared with $72.6 \mathrm{~mm} \mathrm{Hg}$ in the usual care group. Among 2463 analysed patients, there was significantly less exposure to vasopressors in the intervention group, measured by duration of vasopressor infusion and total vasopressor doses expressed in norepinephrine equivalents. Ninetyday mortality in the permissive hypotension and usual care groups was similar ( $41.0 \%$ vs $43.8 \%)$.

Given the lack of advantage associated with higher MAP targets and the lack of harm among elderly patients with MAP targets of $60-65 \mathrm{~mm} \mathrm{Hg}$, the panel recommends targeting a MAP of $65 \mathrm{~mm} \mathrm{Hg}$ in the initial resuscitation of patients with septic shock who require vasopressors.

\section{Admission to intensive care}

Recommendation

10. For adults with sepsis or septic shock who require ICU admission, we suggest admitting the patients to the ICU within $6 \mathrm{~h}$

Weak recommendation, low-quality evidence

\section{Rationale}

The outcome of critically ill patients depends on timely application of critical care interventions in an appropriate environment. Outside the ICU, septic patients are typically seen in the emergency department (ED) and hospital wards. Delayed admissions of critically ill patients from ED are associated with decreased sepsis bundle compliance and increased mortality, ventilator duration, and ICU and hospital length of stay [84]. Data on the optimal time for transfer to the ICU stem from observational studies and registry databases.

In an observational study of 401 ICU patients, authors reported an increase in ICU mortality of $1.5 \%$ for each hour delay of ED to ICU transfer [85]. A retrospective observational study of 14,788 critically ill patients in the Netherlands showed a higher hospital mortality for the higher ED to ICU time quintiles $(2.4-3.7 \mathrm{~h}$ and $>3.7 \mathrm{~h}$ ) compared with the lowest ED to ICU time quintile $(<1.2 \mathrm{~h})$ [86]. When adjusted for severity of illness, an ED to ICU time $>2.4 \mathrm{~h}$ was associated with increased hospital mortality in patients with higher illness severity (ORs of 1.20 (95\% CI 1.03-1.39). Patients with sepsis were not studied separately.

Another study evaluated 50,322 ED patients admitted to 120 US ICUs [87]. Mortality increased when ED stay exceeded 6 h (17\% vs 12.9\%, $p<0.001)$. Among hospital survivors, the delayed admission group had a longer hospital stay, higher mortality, and higher rates of mechanical ventilation and central venous catherisation. Similarly, another study of 12,380 ward patients in $48 \mathrm{UK}$ hospitals showed that [88] delayed admission to ICU led to higher 90-day mortality and further physiological deterioration.

Based on existing data, timely admission of critically ill patients to an ICU environment may result in better patient outcomes. There is also evidence of improved patient satisfaction, increased patient safety, better patient flow and improved staff morale [89]. However, although critical care services are likely best delivered in an ICU environment, there are multiple reasons why immediate transfer of critically ill patients with sepsis to an ICU may not always be possible, in particular in lower and middle income countries (LMIC), where ICU bed availably can be limited. In this case, regular assessment, evaluation, and appropriate treatment should not be delayed, independent of patient location. 


\section{Infection}

\section{Diagnosis of infection}

Recommendation

11. For adults with suspected sepsis or septic shock but unconfirmed infection, we recommend continuously re-evaluating and searching

for alternative diagnoses and discontinuing empiric antimicrobials if an alternative cause of illness is demonstrated or strongly suspected

Best Practice statement

\section{Rationale}

In previous versions of these guidelines, we highlighted the importance of obtaining a full screen for infectious agents prior to starting antimicrobials wherever it is possible to do so in a timely fashion [12, 13]. As a best practice statement, we recommended that appropriate routine microbiologic cultures (including blood) should be obtained before starting antimicrobial therapy in patients with suspected sepsis and septic shock if it results in no substantial delay in the start of antimicrobials (i.e. $<45 \mathrm{~min}$ ). This recommendation has not been updated in this version but remains as valid as before.

The signs and symptoms of sepsis are nonspecific and often mimic multiple other diseases [90-92]. Since there is no "gold standard" test to diagnose sepsis, the bedside provider cannot have a differential diagnosis of sepsis alone in a patient with organ dysfunction. Indeed, a third or more of patients initially diagnosed with sepsis turn out to have non-infectious conditions [90, 93, 94]. Best practice is to continually assess the patient to determine if other diagnoses are more or less likely, especially since a patient's clinical trajectory can evolve significantly after hospital admission, increasing or decreasing the likelihood of a diagnosis of sepsis. With this uncertainty, there can be significant challenges in determining when it is "appropriate" to de-escalate or discontinue antibiotics.

Another major challenge is implementing a system that reminds clinicians to focus on the fact that the patient is still receiving antibiotics each day, especially as providers rotate in and out of the care team. Systems that promote such reassessment by automatic stop orders, electronic prompts, or mandatory check lists all seem useful in theory, but each has disadvantages in terms of provider acceptance or assuring that providers thoughtfully assess the need for antibiotics rather than checking a box in the electronic record or reflexively acknowledging a prompt, without considering its underlying rationale [95].

We did not identify any direct or indirect evidence assessing this important issue. Thus, clinicians are strongly encouraged to discontinue antimicrobials if a non-infectious syndrome (or an infectious syndrome that does not benefit from antimicrobials) is demonstrated or strongly suspected. Since this situation is not always apparent, continued reassessment of the patient should optimise the chances of infected patients receiving antimicrobial therapy and non-infected patients avoiding therapy that is not indicated.

\section{Time to antibiotics}

\section{Recommendations}

12. For adults with possible septic shock or a high likelihood for sepsis, we recommend administering antimicrobials immediately, ideally within $1 \mathrm{~h}$ of recognition

Strong recommendation, low quality of evidence (Septic shock)

Strong recommendation, very low quality of evidence (Sepsis without shock)

13. For adults with possible sepsis without shock, we recommend rapid assessment of the likelihood of infectious versus non-infectious causes of acute illness

Best Practice Statement

\section{Remarks}

Rapid assessment includes history and clinical examination, tests for both infectious and non-infectious causes of acute illness and immediate treatment for acute conditions that can mimic sepsis. Whenever possible this should be completed within $3 \mathrm{~h}$ of presentation so that a decision can be made as to the likelihood of an infectious cause of the patient's presentation and timely antimicrobial therapy provided if the likelihood of sepsis is thought to be high

14. For adults with possible sepsis without shock, we suggest a timelimited course of rapid investigation and if concern for infection persists, the administration of antimicrobials within $3 \mathrm{~h}$ from the time when sepsis was first recognised

Weak recommendation, very low quality of evidence

15. For adults with a low likelihood of infection and without shock, we suggest deferring antimicrobials while continuing to closely monitor the patient.

Weak recommendation, very low quality of evidence

\section{Rationale}

Early administration of appropriate antimicrobials is one of the most effective interventions to reduce mortality in patients with sepsis [96-98]. Delivering antimicrobials to patients with sepsis or septic shock should therefore be treated as an emergency. The imperative to provide antimicrobials as early as possible, however, must be balanced against the potential harms associated with administering unnecessary antimicrobials to patients without infection $[99,100]$. These include a range of adverse events such as allergic or hypersensitivity reactions, kidney injury, thrombocytopenia, Clostridioides difficile infection, and antimicrobial resistance [101-106]. Accurately diagnosing sepsis is challenging as sepsis can present in subtle ways, and some presentations that first appear to be sepsis turn out to be non-infectious conditions [90, 93, 107, 108]. Evaluating the likelihood of infection and severity-of-illness for each patient with suspected sepsis should inform the necessity and urgency of antimicrobials $[99,100]$.

The mortality reduction associated with early antimicrobials appears strongest in patients with septic shock, where a number of studies have reported a strong association between time-to-antibiotics and death in patients with septic shock but weaker associations in patients 
without septic shock $[98,109,110]$. In a study of 49,331 patients treated at 149 New York hospitals, each additional hour of time from ED arrival to administration of antimicrobials was associated with 1.04 increased odds of in-hospital mortality, $p<0.001$ (1.07 (95\% CI 1.05-1.09) for patients receiving vasopressors vs. 1.01 (95\% CI 0.991.04) for patients not on vasopressors) [98]. In a study of 35,000 patients treated at Kaiser Permanente Northern California, each additional hour of time from ER arrival to administration of antimicrobials was associated with 1.09 increased odds of in-hospital mortality (1.07 for patients with "severe" sepsis (lactate $\geq 2$, at least one episode of hypotension, required non-invasive or invasive mechanical ventilation or has organ dysfunction) and 1.14 for patients with septic shock); which equated to a $0.4 \%$ absolute mortality increase for "severe" sepsis and a $1.8 \%$ absolute increase for septic shock [110]. Finally, in a study of 10,811 patients treated in four Utah hospitals, each hour delay in time from ED arrival to administration of antimicrobials was associated with 1.16 increased odds of in-hospital and 1.10 increased odds of 1-year mortality (1.13 in patients with hypotension vs 1.09 in patients without hypotension) [111]. Other studies, however, did not observe an association between antimicrobial timing and mortality [112-117].

It should be noted that all the aforementioned studies were observational analyses and hence at risk of bias due to insufficient sample size, inadequate risk-adjustment, blending together the effects of large delays until antibiotics with short delays, or other study design issues [118].

In patients with sepsis without shock, the association between time to antimicrobials and mortality within the first few hours from presentation is less consistent [98, 110]. Two RCTs have been published [119, 120]. One failed to achieve a difference in time-to-antimicrobials between arms [120]. The other found no significant difference in mortality despite a 90-min difference in median time interval to antimicrobial administration [119]. Observational studies do, however, suggest that mortality may increase after intervals exceeding 3-5 h from hospital arrival and/or sepsis recognition [98, 111, $119,120]$. We therefore suggest initiating antibiotics in patients with possible sepsis without shock as soon as sepsis appears to be the most likely diagnosis, and no later than $3 \mathrm{~h}$ after sepsis was first suspected if concern for sepsis persists at that time.

Overall, given the high risk of death with septic shock and the strong association of antimicrobial timing and mortality, the panel issued a strong recommendation to administer antimicrobials immediately, and within $1 \mathrm{~h}$, in all patients with potential septic shock. In addition, for patients with confirmed/very likely sepsis, we recommend antimicrobials be administered immediately
(Fig. 1). For patients with possible sepsis without shock, we recommend a rapid assessment of infectious and non-infectious etiologies of illness be undertaken to determine, within $3 \mathrm{~h}$, whether antibiotics should be administered or whether antibiotics should be deferred while continuing to monitor the patient closely.

Limited data from resource-limited settings suggest that timely administration of antimicrobials in patients with sepsis and septic shock is beneficial and potentially feasible [121126]. Access and availability of a wide range of antimicrobials in such settings may however vary $[54,55,57,59,61]$. The availability and turn-around time for laboratory testing, rapid infectious diagnostic, imaging, etc. varies widely by regions and settings. As such, the rapid assessment of infectious and non-infectious etiologies of illness will differ across settings, depending on what is feasible to achieve. Recent recommendations pertaining to the use of antimicrobials in patients with sepsis and septic shock in resource-limited settings are in line with the current recommendations [123].

\section{Biomarkers to start antibiotics \\ Recommendation \\ 16. For adults with suspected sepsis or septic shock, we suggest against using procalcitonin plus clinical evaluation to decide when to start antimicrobials, as compared to clinical evaluation alone \\ Weak recommendation, very low quality of evidence}

\section{Rationale}

Procalcitonin is undetectable in healthy states, but rises rapidly in response to pro-inflammatory stimuli, especially bacterial infections [127]. In theory, procalcitonin levels in combination with clinical evaluation may facilitate the diagnosis of serious bacterial infections and prompt early initiation of antimicrobials. In a meta-analysis of 30 studies (3244 patients), procalcitonin had a pooled sensitivity of $77 \%$ and specificity of $79 \%$ for sepsis in critically ill patients [128].

We identified direct evidence from three RCTs that compared procalcitonin-guided protocols for antibiotic initiation vs usual care [129-131]. A meta-analysis of the three trials ( $n=1769$ ICU patients) found no difference in shortterm mortality (RR 0.99; 95\% CI 0.86-1.15), length of ICU stays (MD 0.19 days; $95 \% \mathrm{CI}-0.98$ to 1.36 ) or length of hospitalisation (MD 7.00 days; $95 \% \mathrm{CI}-26.24$ to 12.24 ). Long-term mortality, readmission rates and hospital-free days were not reported in any of the trials, and no relevant studies on the costs associated with use of procalcitonin were found. In general, knowledge about the undesirable effects was lacking, and the quality of evidence was very low. Published guidelines for the management of community-acquired pneumonia recommend initiation of antimicrobials for patients with community-acquired pneumonia regardless of procalcitonin level [132]. 


\title{
Antibiotic Timing
}

\author{
Shock is present
}

Shock is absent

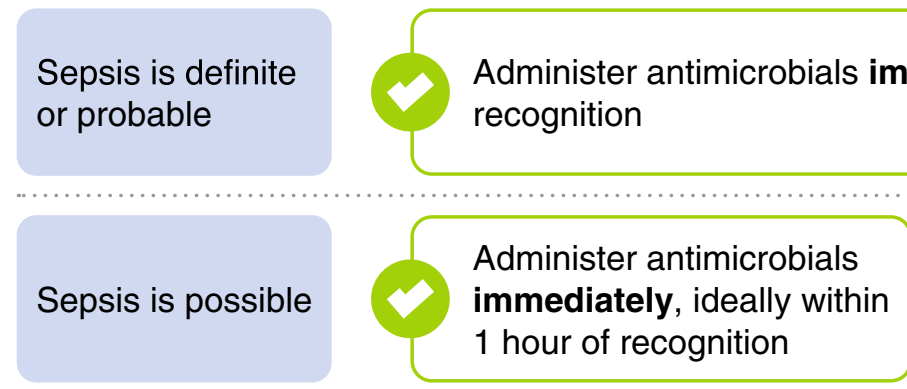

Rapid assessment* of infectious vs noninfectious causes of acute illness

Administer antimicrobials within $\mathbf{3}$ hours if concern for infection persists

\begin{abstract}
${ }^{*}$ Rapid assessment includes history and clinical examination, tests for both infectious and non-infectious causes of acute illness and immediate treatment for acute conditions that can mimic sepsis. Whenever possible this should be completed within 3 hours of presentation so that a decision can be made as to the likelihood of an infectious cause of the patient's presentation and timely antimicrobial therapy provided if the likelihood is thought to be high.
\end{abstract}

Fig. 1 Recommendations on timing of antibiotic administration

With no apparent benefit, unknown costs, and limited availability in some settings including low- and middleincome countries (LMICs), the panel issued a weak recommendation against using procalcitonin to guide antimicrobial initiation in addition to clinical evaluation.

\section{Antimicrobial choice}

\section{Recommendations}

17. For adults with sepsis or septic shock at high risk of methicillin resistant staph aureus (MRSA), we recommend using empiric antimicrobials with MRSA coverage over using antimicrobials without MRSA coverage Best Practice statement

18. For adults with sepsis or septic shock at low risk of methicillin resistant staph aureus (MRSA), we suggest against using empiric antimicrobials with MRSA coverage, as compared with using antimicrobials without MRSA coverage

Weak recommendation, low quality of evidence

\section{Rationale}

The decision on whether to include an antibiotic active against MRSA in an empiric treatment regimen for sepsis and septic shock depends upon (a) the likelihood that the patient's infection is caused by MRSA, (b) the risk of harm associated with withholding treatment for MRSA in a patient with MRSA, and (c) the risk of harm associated with MRSA treatment in a patient without MRSA.

MRSA accounts for approximately $5 \%$ of culture-positive infections among critically ill patients [133], and may be decreasing according to some reports [134, 135]. The incidence of MRSA varies, however, by region (ranging from $\sim 2 \%$ in Western Europe to $10 \%$ in North America) and by patient-related characteristics [133, 136, 137]. Patient-related risk factors for MRSA include prior history of MRSA infection or colonisation, recent IV antibiotics, history of recurrent skin infections or chronic wounds, presence of invasive devices, haemodialysis, recent hospital admissions and severity of illness [136, 138-142].

Observational data on the impact of including MRSA coverage in empiric regimens vary. Some studies focus on patients with documented MRSA infections, while others evaluate the impact of MRSA coverage in undifferentiated patients. Among patients with documented MRSA infections, delays of $>24-48 \mathrm{~h}$ until antibiotic administration are associated with increased mortality in some studies [143-147], but not in others [148-154]. Among undifferentiated patients with pneumonia or sepsis, 
broad-spectrum regimens including agents active against MRSA were associated with higher mortality, particularly among patients without MRSA [137, 151, 155, 156]. The undesirable effects associated with unnecessary MRSA coverage are also supported by studies showing an association between early discontinuation of MRSA coverage and better outcomes in patients with negative nares or bronchoalveolar lavage (BAL) MRSA PCR [157-159].

Failure to cover for MRSA in a patient with MRSA may be harmful, but unnecessary MRSA coverage in a patient without MRSA may also be harmful. Data from RCTs, including the evaluation of nasal swab testing to withhold therapy for MRSA, are warranted, and studies on rapid diagnostic tools and clinical prediction rules for MRSA are needed.

Recommendations
19. For adults with sepsis or septic shock and high risk for multidrug
resistant (MDR) organisms, we suggest using two antimicrobials with
gram-negative coverage for empiric treatment over one gram-negative
agent
Weak recommendation, very low quality of evidence
20. For adults with sepsis or septic shock and low risk for MDR organisms,
we suggest against using two Gram-negative agents for empiric
treatment, as compared to one Gram-negative agent
Weak recommendation, very low quality of evidence
21. For adults with sepsis or septic shock, we suggest against using
double gram-negative coverage once the causative pathogen and the
susceptibilities are known
Weak recommendation, very low quality of evidence

\section{Rationale}

Considering the increasing frequency of MDR bacteria in many parts of the world and associations between delays in active therapy and worse outcomes, the initial use of multidrug therapy is often required to ensure the empiric regimen includes at least one effective agent that is active against the offending organism [12, 13]. In the empiric phase-before causative agent(s) and susceptibilities are known, the optimal choice of antibiotic therapy depends on the local prevalence of resistant organisms, patient risk factors for resistant organisms, and the severity of illness. In the directed/targeted phase, once causative agent(s) and susceptibilities are known, sustained double gram-negative coverage is rarely necessary except for patients with highly resistant organisms.

This was borne out in a recent systematic review with meta-analysis of $10 \mathrm{RCTs}$, no differences in mortality or other patient-important outcomes between empiric mono- vs. combination antibiotic therapy in adult ICU patients with severe sepsis or septic shock were observed, also when taking disease severity into consideration
[160]. Results from the largest RCT included in the metaanalysis (a comparison of sustained courses of moxifloxacin and meropenem vs meropenem alone in a low endemic resistance setting) were consistent with the findings from the meta-analysis [161].

Recommendations about the use of more than one gram-negative agent for empiric treatment over one gram-negative agent are challenging given clinical heterogeneity, including patient characteristics, source of infection, causative agents, and antibiotic resistance patterns. Local information about the resistance patterns of the most common causative agents of sepsis is essential to choose the most appropriate empiric antibiotic therapy. For this reason, we refrained from proposing recommendations regarding double gram-negative coverage in patients with sepsis or septic shock overall, but instead recommend tailoring the use of double coverage based on patients' risk of MDR pathogens. Factors to guide this decision include: proven infection or colonisation with antibiotic-resistant organisms within the preceding year, local prevalence of antibiotic-resistant organisms, hospital-acquired/healthcare associated (versus communityacquired infection), broad-spectrum antibiotic use within the preceding 90 days, concurrent use selective digestive decontamination (SDD), travel to a highly endemic country within the preceding 90 days (see https://resistance map.cddep.org/) and hospitalisation abroad within the preceding 90 days [162-164]. In the directed/targeted phase, once causative agent(s) and susceptibilities are known, sustained double gram-negative coverage is not necessary except possibly for patients with highly resistant organisms with no proven safe and efficacious therapeutic option.

The overall quality of evidence was very low, and the direct costs of antibiotics can increase with the routine use of multiple agents for treatment. This may specifically have an impact in resource-limited settings.

In general, in patients at high risk for MDR organisms, we suggest using two gram negative agents for empiric treatment to increase the likelihood of adequate coverage, while in patients with a low risk for MDR organisms, we suggest using a single agents for empiric treatment, as there are no apparent benefits of using two agents and the a risk of antimicrobial-associated undesirable effects, including direct toxicity, Clostridioides difficile infection and development of antibiotic resistance [165]. Empiric double coverage of gram-negative bacilli is most important in patients at high risk for resistant organisms with severe illness, particularly septic shock. 


\section{Antifungal therapy}

Recommendations

22. For adults with sepsis or septic shock at high risk of fungal infec-

tion, we suggest using empiric antifungal therapy over no antifungal therapy

Weak recommendation, low quality of evidence

23. For adults with sepsis or septic shock at low risk of fungal infection we suggest against empiric use of antifungal therapy

Weak recommendation, low quality of evidence

\section{Rationale}

Sepsis and septic shock due to fungi are most commonly observed in ICUs and are associated with poor outcomes [166-170]. Some observational studies suggested that prompt initiation of appropriate empiric antifungal therapy may be associated with a reduction in mortality, however these studies do not prove a causal relationship between antifungal therapy and outcome, nor do they clarify the role of timing of treatment, and some other studies have failed to show this association [167, 171-173].

In an updated meta-analysis of empiric antifungal therapy versus no antifungal therapy in adult critically ill patients, no difference in short-term mortality was observed. In the largest and most recent $\mathrm{RCT}-$ EMPIRICUS - there was also no difference in outcome between patients receiving empiric antifungal therapy (micafungin) and patients receiving placebo [174]. The overall quality of evidence was low, and treatment with empiric antifungals may be associated with increased costs.

While patients with sepsis or septic shock may not in general benefit from empiric antifungals, some patients with particular risk factors for fungal infection may, for example patients with febrile neutropenia who fail to defervesce after 4-7 days of broad-spectrum antibacterial therapy are at increased risk of having fungal disease (Table 2) $[175,176]$. The risk of Candida sepsis or septic shock for other immunosuppressed populations is highly disease- and therapy-specific. Importantly, the decision to start empiric antifungal therapy depends on the type and number of risk factors, along with the local epidemiology of fungal infections.

Accordingly, we suggest using empiric antifungal therapy in patients at high risk of fungal infection, while we suggest avoiding this if the risk is low. The choice of antifungal agent for empiric therapy depends on multiple issues including host factors, prior colonisation and infection, prior exposure to prophylactic or therapeutic antifungal therapy, comorbidities, and the toxicities and drug interactions of the therapeutic options.
Antiviral therapy

Recommendation

24. We make no recommendation on the use of antiviral agents

\section{Rationale}

Viral infections encompass a broad spectrum of pathogens and diseases in humans but-apart from specific clinical situations such as epidemics/pandemics-are rarely the primary cause of sepsis. In a recent large international point prevalence study, viruses were documented in less than $4 \%$ of infections [133].

Historically, influenza has been one of the more common viral causes of sepsis. However, it is unclear to what extent the primary viral infection as opposed to bacterial pneumonia co-infection is the cause of organ dysfunction in these patients [219-222]. More recently, SARS-CoV-2 (causing COVID-19) is now responsible for many cases of infection and sepsis [223]. The ongoing pandemic due to SARS-CoV-2 has resulted in the understanding of this condition changing very rapidly [224].

While there appears to be no overall effect of neuraminidase inhibitors on mortality in patients with influenza-related pneumonia, there may be an effect when administered early in the course of the disease [225]. For detailed information on specific antiviral therapy, including for influenza and SARS CoV-2, please refer to dedicated clinical practice guidelines [226-228].

Immunocompromised patients are particularly vulnerable to viral infections, including patients with neutropenia, human immunodeficiency virus (HIV) infection, haematological malignancies and haematopoietic stem cell transplantation or solid organ transplants; in these patients herpes simplex virus, Epstein-Barr virus, cytomegalovirus, and respiratory viruses such as adenoviruses, can cause severe disease [229]. Tropical and subtropical regions have endemic and epidemic outbreaks of zoonotic viral infections including those caused by Dengue, Ebola, Lassa, Marburg, Sin Nombre and Chikungunya virus. Many of these can manifest with clinical signs of sepsis, particularly in their early stages. Unfortunately, effective therapies are lacking for most of these viruses.

The desirable effects of empiric antiviral therapy are unknown, and as for other antimicrobial agents there is a risk of undesirable effects [165]. Data on cost effectiveness were not available.

Due to the rapidly changing position related to antiviral therapies in critically ill patients presenting with several acute respiratory failure, this panel decided not to issue a recommendation on antiviral therapies and to refer the reader to more specific guidelines [226]. 


\section{Delivery of antibiotics}

Recommendation

25. For adults with sepsis or septic shock, we suggest using prolonged infusion of beta-lactams for maintenance (after an initial bolus) over conventional bolus infusion

Weak recommendation, moderate quality of evidence

\section{Rationale}

Beta-lactam antibiotics may be subject to changes in important pharmacokinetic parameters in the setting of sepsis and septic shock resulting in sub-therapeutic concentrations [230, 231]. As opposed to conventional intermittent infusion (infusion $\leq 30 \mathrm{~min}$ ), administration by prolonged IV infusion, either as an extended infusion (antibiotic infused over at least half of the dosing interval) or as a continuous infusion, results in sustained beta-lactam concentrations which align with the pharmacodynamics of these drugs.

Two meta-analyses reported similar results supporting reduced short-term mortality (RR 0.70; 95\% CI 0.570.87 ) with prolonged infusion of beta-lactams [232, 233].

No trials assessed the undesirable effects of continuous infusion, and the desirable effects were deemed important, while the overall quality of evidence was moderate. Prolonged infusion is a feasible intervention if suitable IV access is present, and resources are available to ensure the beta-lactam is infused over the necessary duration. The latter may be an issue in some resource limited settings, including LMICs.

Administration of a loading dose of antibiotic before prolonged infusion is essential to avoid delays to achieving effective beta-lactam concentrations [234]. Over the course of therapy, both extended and continuous infusions will occupy a venous catheter/lumen more than an intermittent infusion and drug-stability and drug-drug compatibility considerations are important to ensure effectiveness of antibiotic and other IV drug therapies [235].

The reduction in short-term mortality from prolonged infusion of beta-lactams is significant with the intervention being feasible with negligible cost implications and no data suggesting inferior outcomes with prolonged infusion. Accordingly, we suggest prolonged infusion of beta-lactams over conventional bolus infusion in patients with sepsis and septic shock if the necessary equipment is available. Further research is needed on long-term outcomes, on the effect on emergence of antimicrobial resistance, and on costs of prolonged versus bolus infusion of beta-lactams [236].

\section{Pharmacokinetics and pharmacodynamics}

Recommendation

26. For adults with sepsis or septic shock, we recommend optimising dosing strategies of antimicrobials based on accepted pharmacokinetic/pharmacodynamic (PK/PD) principles and specific drug properties

Best Practice Statement

\section{Rationale}

Antibiotics are subject to changes in PK/PD parameters in sepsis and septic shock where resultant concentrations may be too low risking clinical failure, or too high leading to toxicity (Table 3) [237-239]. Augmented renal clearance [240], AKI [241], hypoalbuminemia [242], RRT [243, 244], and extracorporeal membrane oxygenation $[245,246]$ are examples of common scenarios that affect the concentrations of some antibiotics. Administration of antibiotics using an approach that adheres to PK/PD principles and using dosing regimens developed in patients with sepsis and septic shock is more likely to result in effective and safe drug concentrations compared to use of dosing recommendations provided in the manufacturer's product information [247].

We did not identify any relevant data quantifying the value of dosing based on PK/PD principles in adults with sepsis and septic shock. Although there are no data on this topic directly derived from adults with sepsis and septic shock, data from a broader patient population, critically ill patients, support an increased likelihood of achieving effective and safe antibiotic concentrations when applying PK/PD principles to dosing [248]. The application of PK/ PD principles can be aided by clinical pharmacists [249]. Some studies in critically ill patients have reported benefits in terms of clinical cure [237, 250-253].

Applying a $\mathrm{PK} / \mathrm{PD}$ approach to antibiotic dosing requires support from knowledgeable clinician team members [254], use of a patient population-specific guideline document [255], use of therapeutic drug monitoring [256], and/or use of dosing software [238, 248]. Some of these potential approaches to application of PK/ PD-based dosing require extra resources, some of which may not be available in all settings, in which case freely available resources such as dosing nomograms can be used [234, 257, 258]. Guidance on how to apply a PK/PD approach for specific drug classes have been described elsewhere [237]. Further research is needed on short- and long-term mortality outcomes, effect on emergence of antimicrobial resistance, impact on drug stability within prolonged infusions and health economics of different PK/PD-based approaches to dosing (see Table 3). 


\section{Table 1 Table of current recommendations and changes from the previous 2016 recommendations}

\section{TABLE OF RECOMMENDATIONS}

NEW and UPDATED recommendations are highlighted with a blue background

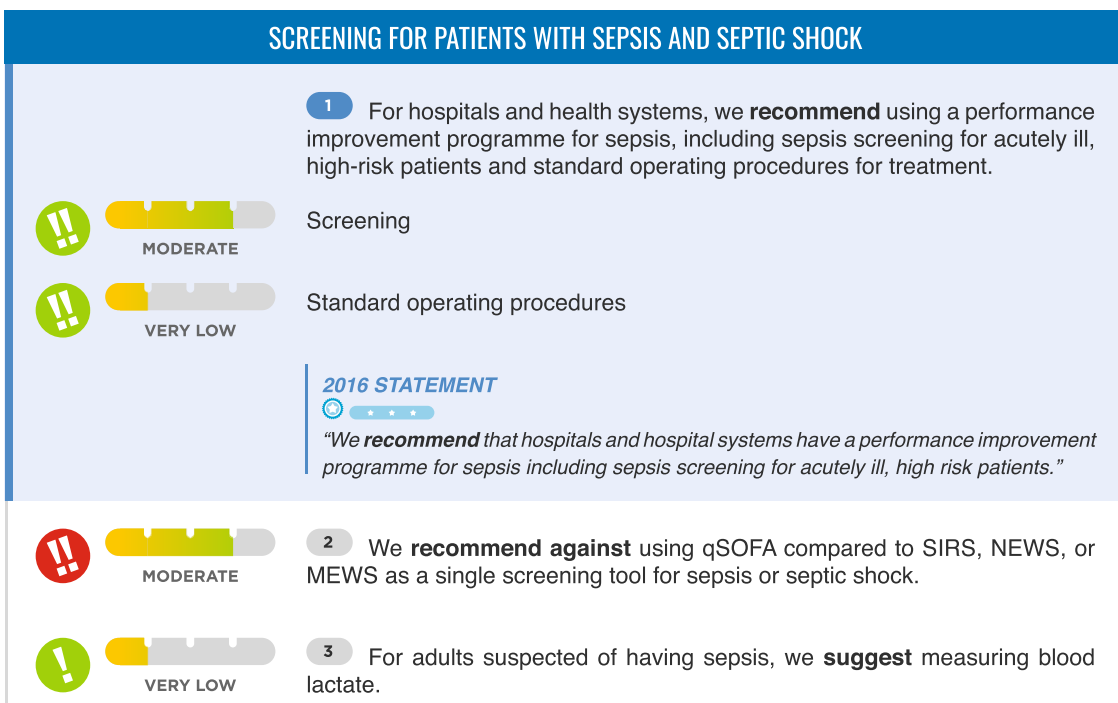

\section{INITIAL RESUSCITATION}

Sepsis and septic shock are medical emergencies, and we recommend that treatment and resuscitation begin immediately.

For patients with sepsis induced hypoperfusion or septic shock we suggest that at least $30 \mathrm{~mL} / \mathrm{kg}$ of intravenous (IV) crystalloid fluid should be given within the first 3 hours of resuscitation.

\section{STATEMENT}

$\downarrow$ (1)

"We recommend that in the initial resuscitation from sepsis-induced hypoperfusion, at least $30 \mathrm{ml} / \mathrm{kg}$ of intravenous crystalloid fluid be given within the first 3 hours."

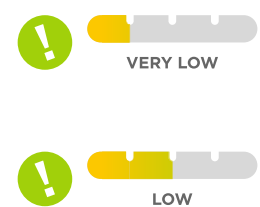

6 For adults with sepsis or septic shock, we suggest using dynamic measures to guide fluid resuscitation, over physical examination, or static parameters alone.

7 For adults with sepsis or septic shock, we suggest guiding resuscitation to decrease serum lactate in patients with elevated lactate level, over not using serum lactate.

For adults with septic shock, we suggest using capillary refill time to guide resuscitation as an adjunct to other measures of perfusion.

\section{MEAN ARTERIAL PRESSURE}

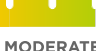

9 For adults with septic shock on vasopressors, we recommend an initial MODERATE target mean arterial pressure (MAP) of $65 \mathrm{~mm} \mathrm{Hg}$ over higher MAP targets.
I

I NO RECOMMENDATION

I WEAK RECOMMENDATION

1 STRONG RECOMMENDATION

\section{0}

I WEAK RECOMMENDATION

AGAINST

1 II

I STRONG RECOMMENDATION I AGAINST

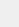

(1)

I HIGH QUALITY EVIDENCE

I MODERATE QUALITY EVIDENCE

I

I LOW QUALITY EVIDENCE

I

I VERYLOW QUALITY EVIDENCE

I

I 4 UPGRADE

$\downarrow$ DOWNGRADE

I

NO CHANGE FROM

PREVIOUS GUIDELINES

I

I

I

NEW / CHANGED

RECOMMENDATION 
Table 1 (continued)

\section{ADMISSION TO INTENSIVE CARE}

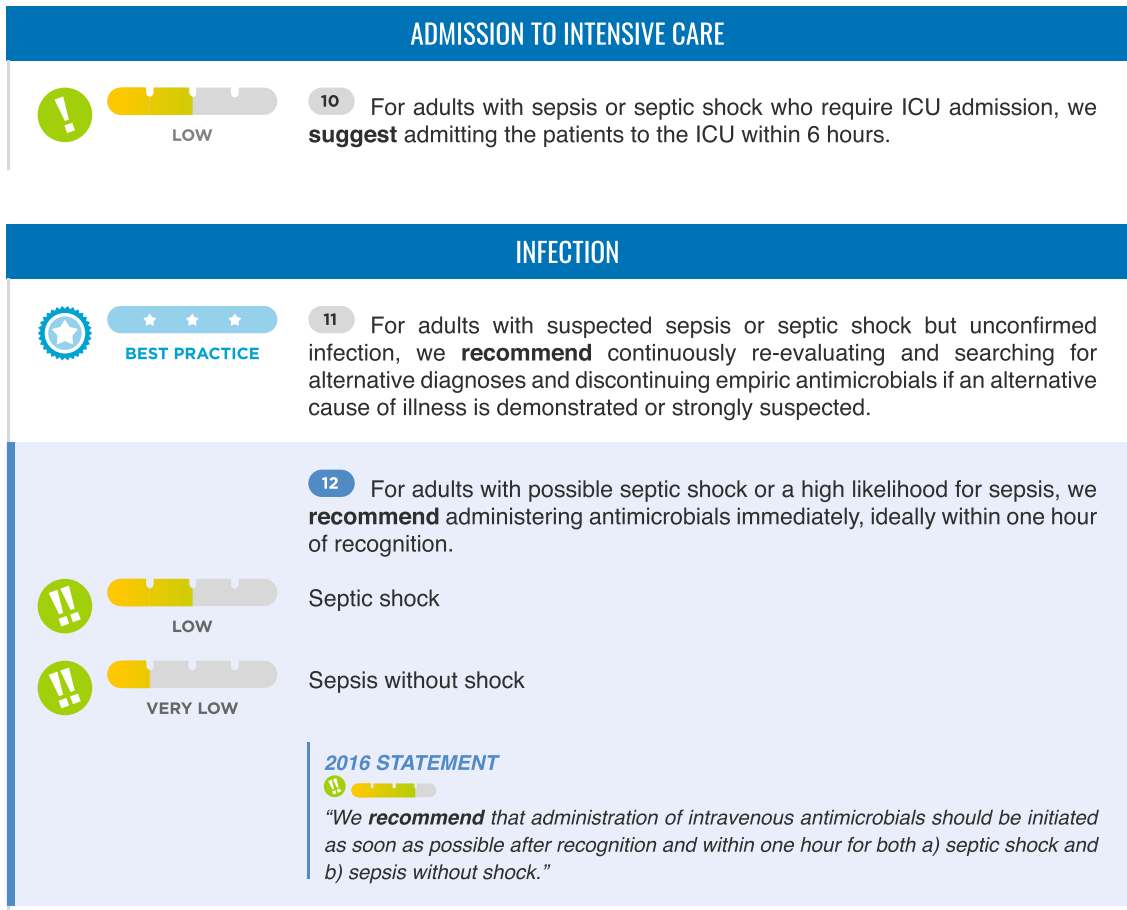

t 13 For adults with possible sepsis without shock, we recommend rapid BEST PRACTICE assessment of the likelihood of infectious versus non-infectious causes of acute illness.

\section{For adults with possible sepsis without shock, we suggest a time- VERY LOW limited course of rapid investigation and if concern for infection persists, the administration of antimicrobials within 3 hours from the time when sepsis was first recognized. \\ 2016 STATEMENT \\ (19) \\ "We recommend that administration of intravenous antimicrobials should be initiated as soon as possible after recognition and within one hour for both a) septic shock and b) sepsis without shock." \\ 15 For adults with a low likelihood of infection and without shock, we suggest deferring antimicrobials while continuing to closely monitor the patient. \\ 2016 STATEMENT \\ (1) \\ "We recommend that administration of intravenous antimicrobials should be initiated as soon as possible after recognition and within one hour for both a) septic shock and b) sepsis without shock."}

16 For adults with suspected sepsis or septic shock, we suggest against using procalcitonin plus clinical evaluation to decide when to start antimicrobials, as compared to clinical evaluation alone. 
Table 1 (continued)

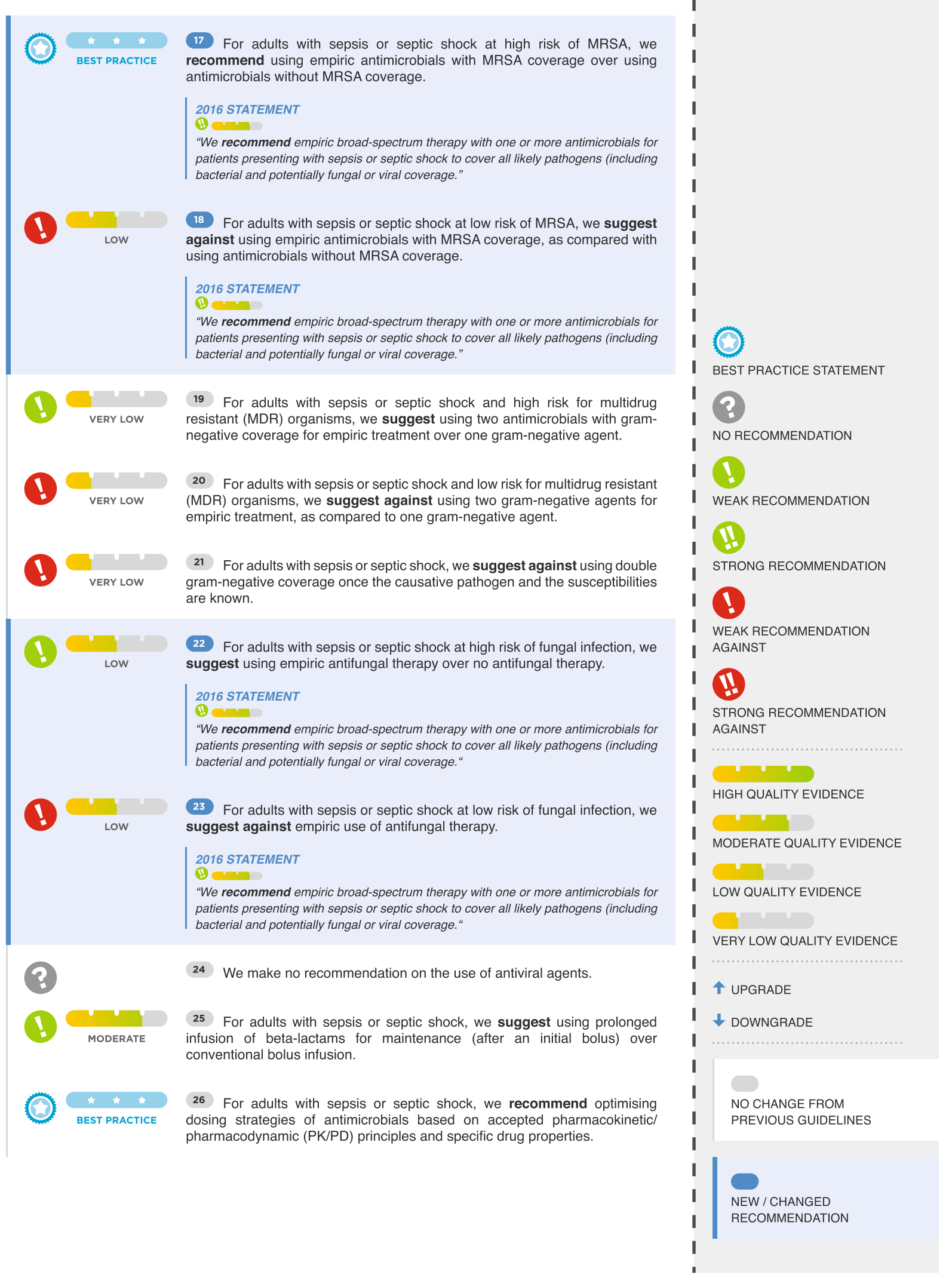


Table 1 (continued)

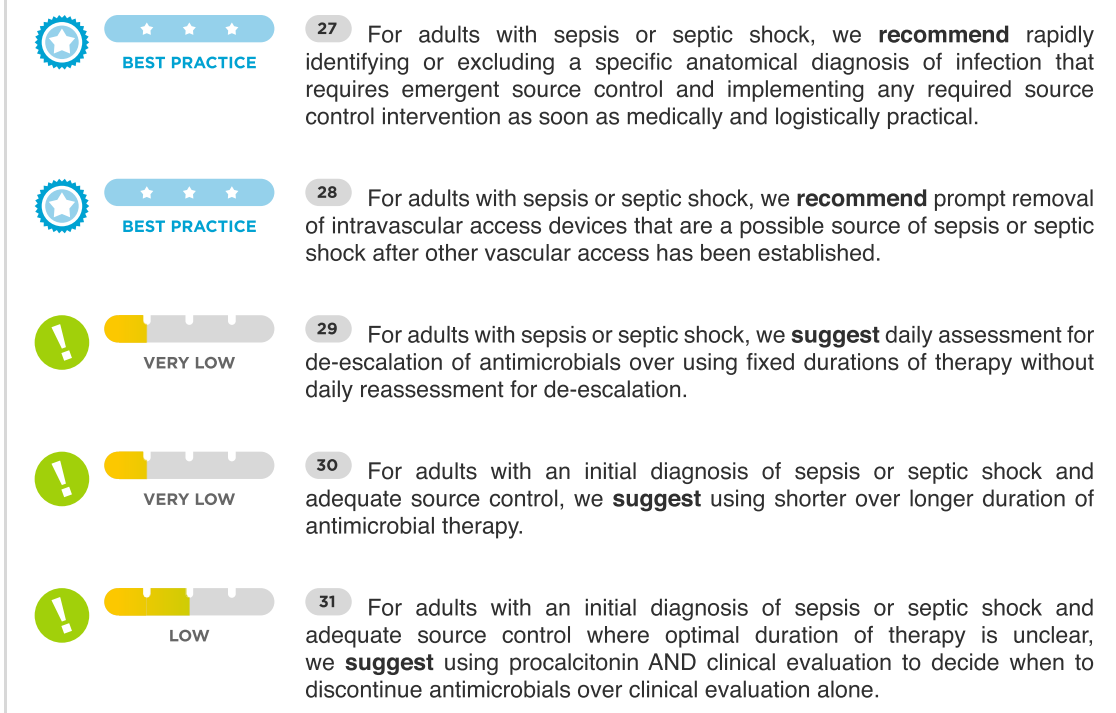

\section{HEMODYNAMIC MANAGEMENT}

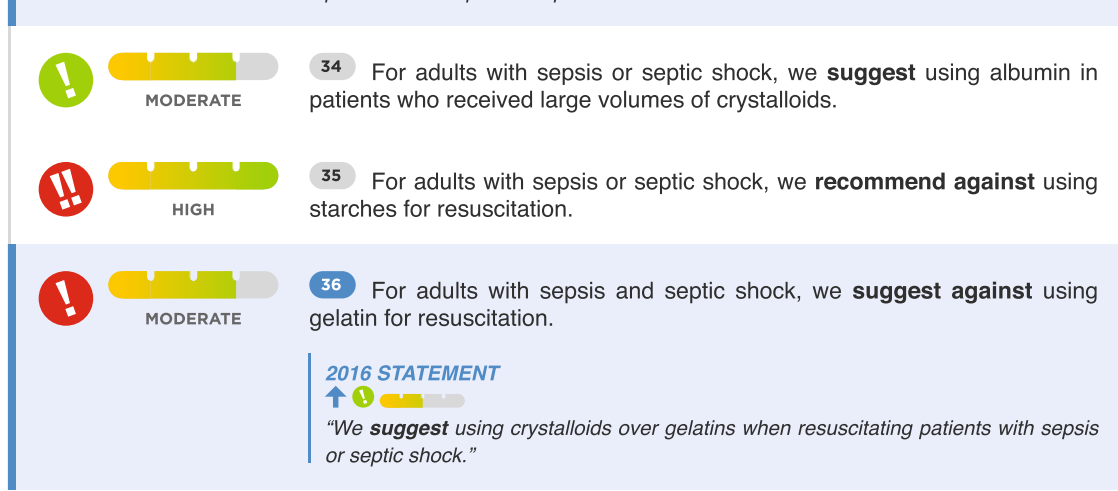

32 For adults with sepsis or septic shock, we recommend using crystalloids as first-line fluid for resuscitation.

33 Fow adults with sepsis or septic shock, we suggest using balanced
crystalloids instead of normal saline for resuscitation.
$\begin{aligned} & 2016 \text { STATEMENT } \\ & \text { "We suggest using either balanced crystalloids or saline for fluid resuscitation of } \\ & \text { patients with sepsis or septic shock" }\end{aligned}$

\section{11}

STRONG RECOMMENDATION IGAINST

I

I

I HIGH QUALITY EVIDENCE

I MODERATE QUALITY EVIDENCE I COY

I LOW QUALITY EVIDENCE

I

I VERY LOW QUALITY EVIDENCE I

I $\uparrow$ UPGRADE

I DOWNGRADE

I

I

I

NO CHANGE FROM PREVIOUS GUIDELINES

I

I

I

I I 
Table 1 (continued)

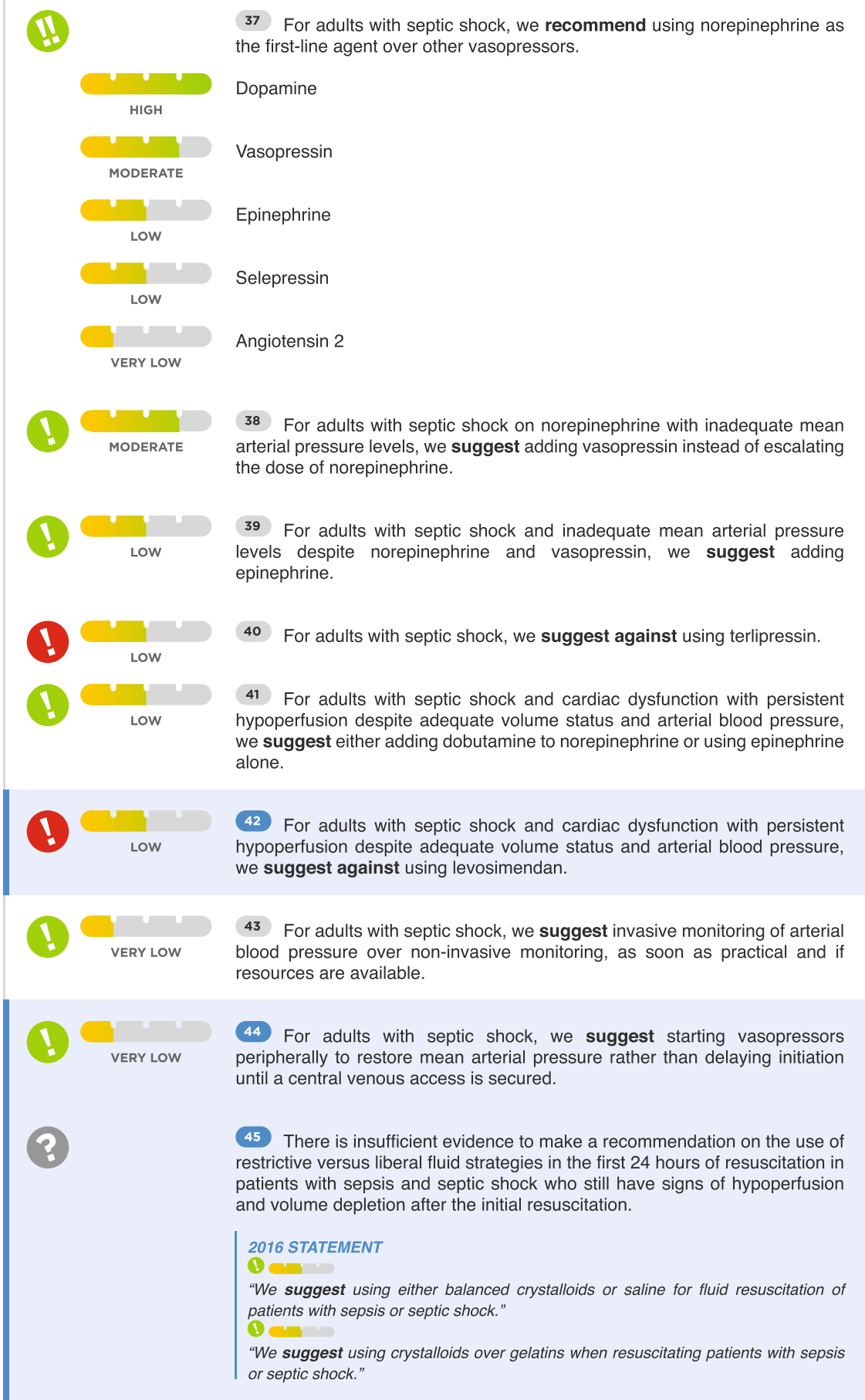

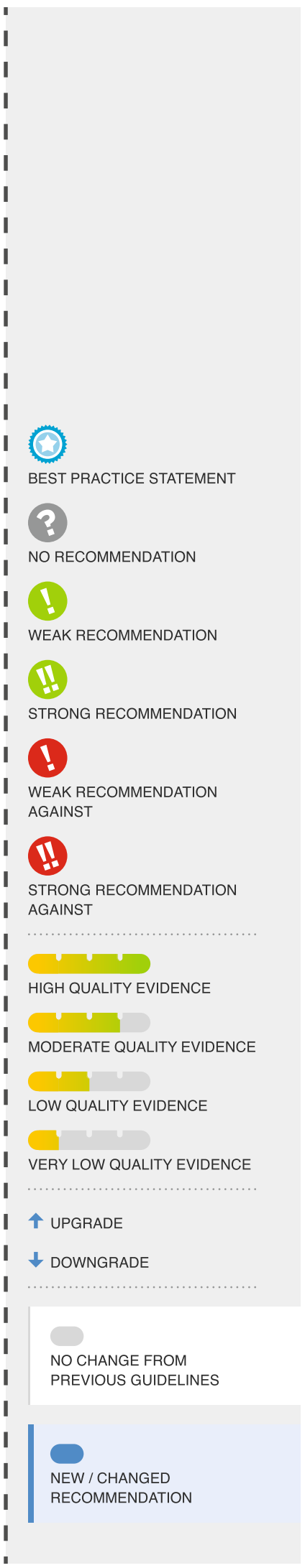


Table 1 (continued)

VENTILATION

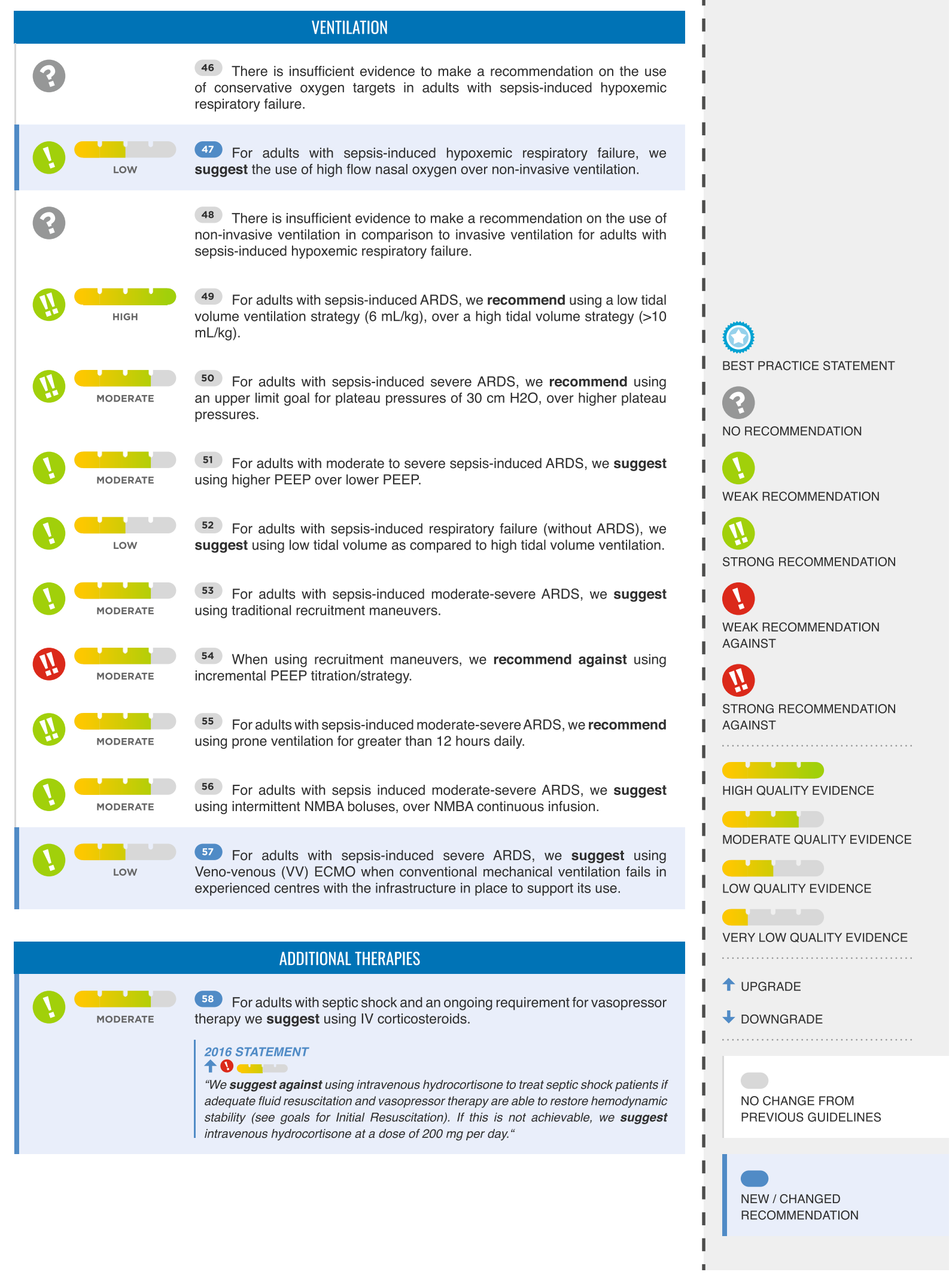


Table 1 (continued)

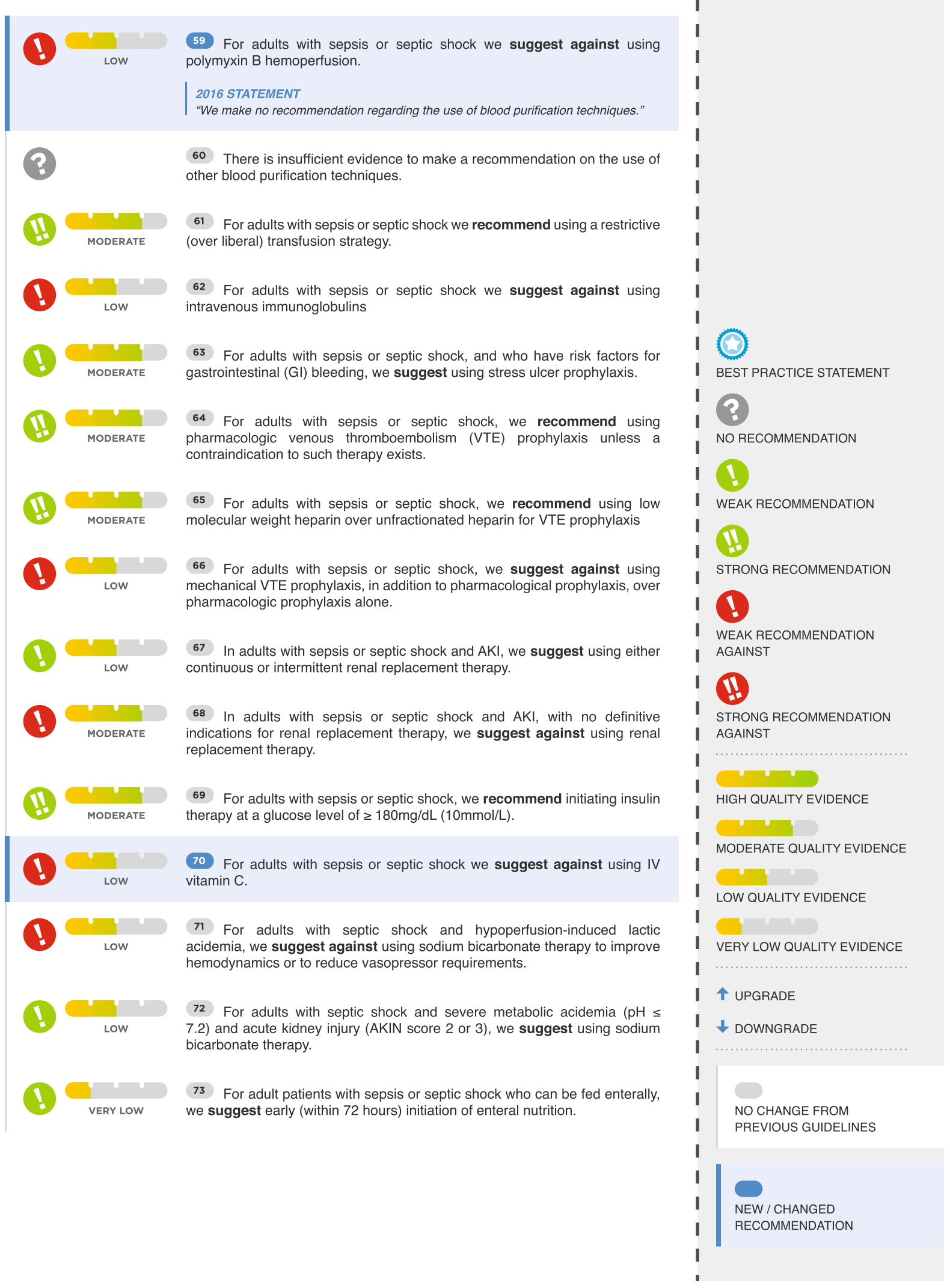


Table 1 (continued)

LONG-TERM OUTCOMES AND GOALS OF CARE

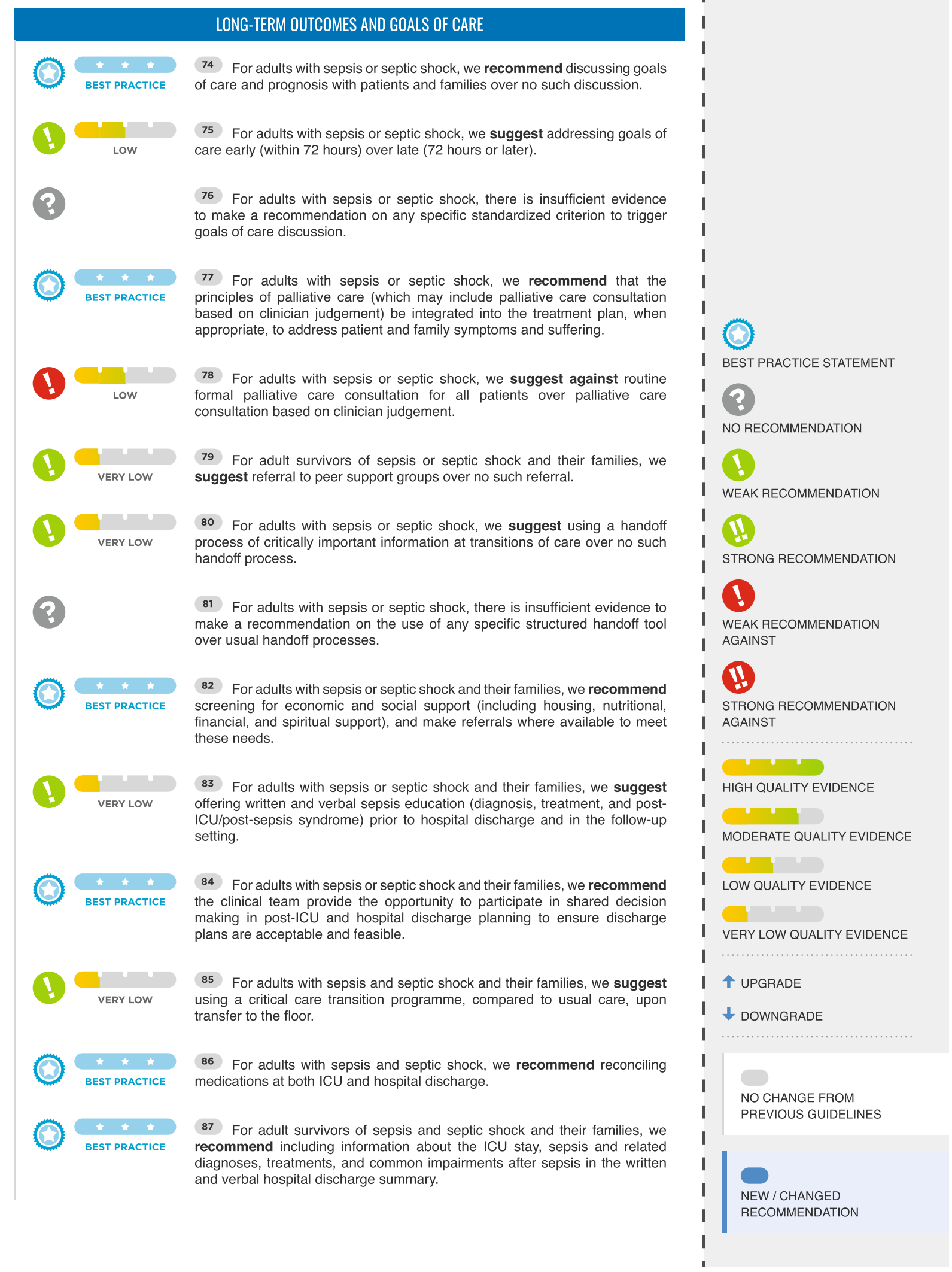


Table 1 (continued)

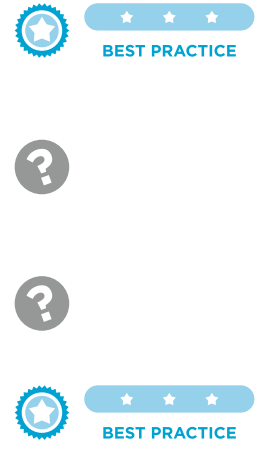

BEST PRACTIC

VERY LOW

ERY LOW
88 For adults with sepsis or septic shock who developed new impairments, we recommend hospital discharge plans include follow-up with clinicians able to support and manage new and long-term sequelae.

89 For adults with sepsis or septic shock and their families, there is insufficient evidence to make a recommendation on early post-hospital discharge follow-up compared to routine post-hospital discharge follow-up.

90 For adults with sepsis or septic shock, there is insufficient evidence to make a recommendation for or against early cognitive therapy.

91 For adult survivors of sepsis or septic shock, we recommend assessment and follow-up for physical, cognitive, and emotional problems after hospital discharge.

22 For adult survivors of sepsis or septic shock, we suggest referral to a post-critical illness follow-up programme if available.

93 For adult survivors of sepsis or septic shock receiving mechanical ventilation for $>48$ hours or an ICU stay of $>72$ hours, we suggest referral to a post-hospital rehabilitation programme. NO RECOMMENDATION

I

I

I

I STRONG RECOMMENDATION

1

I WEAK RECOMMENDATION

I AGAINST

10

STRONG RECOMMENDATION AGAINST

I

I

I प M

HIGH QUALITY EVIDENCE

I MODERATE QUALITY EVIDENCE

I LOW QUALITY EVIDENCE

I

I VERY LOW QUALITY EVIDENCE

I

I $\uparrow$ UPGRADE

I $\downarrow$ DOWNGRADE

|

I

I

NO CHANGE FROM PREVIOUS GUIDELINES

I

I

NEW / CHANGED RECOMMENDATION 
Use of therapeutic drug monitoring has been described for all drugs, although it is not widely available for most.

\section{Source control}

Recommendation

27. For adults with sepsis or septic shock, we recommend rapidly identifying or excluding a specific anatomical diagnosis of infection that requires emergent source control and implementing any required source control intervention as soon as medically and logistically practical

Best Practice Statement

\section{Rationale}

Appropriate source control is a key principle in the management of sepsis and septic shock [12, 13]. Source control may include drainage of an abscess, debriding infected necrotic tissue, removal of a potentially infected device, or definitive control of a source of ongoing microbial contamination [262]. Foci of infection readily amenable to source control include intra-abdominal abscesses, gastrointestinal perforation, ischaemic bowel or volvulus, cholangitis, cholecystitis, pyelonephritis associated with obstruction or abscess, necrotizing soft tissue infection, other deep space infection (e.g., empyema or septic arthritis), and implanted device infections [262].

Source control of infectious foci was associated with improved survival in recent observational and cluster randomised studies [120, 263, 264]. Source control should be achieved as soon as possible following initial resuscitation $[265,266]$. While there are limited data to conclusively issue a recommendation regarding the timeframe in which source control should be obtained, smaller studies suggest that source control within 6-12 h is advantageous [265-271]. Studies generally show reduced survival beyond that point. The failure to show benefit with source control implemented in less than $6 \mathrm{~h}$ may be a consequence of the limited number of patients and the heterogeneity of the intervention. Therefore, any required source control intervention in sepsis and septic shock should ideally be implemented as soon as medically and logistically practical after the diagnosis is made [120]. Clinical experience suggests that without adequate source control, many severe presentations will not stabilise or improve despite rapid resuscitation and provision of appropriate antimicrobials. In view of this fact, prolonged efforts at medical stabilisation in lieu of source control for severely ill patients, particularly those with septic shock, are generally not advised [272].

The selection of optimal source control methods must weigh the benefits and risks of the specific intervention, the patient's preference, clinician's expertise, availability, risks of the procedure, potential delays, and the probability of the procedure's success. In general, the least invasive option that will effectively achieve source control should be pursued.
Open surgical intervention should be considered when other interventional approaches are inadequate or cannot be provided in a timely fashion. Surgical exploration may also be indicated when diagnostic uncertainty persists despite radiologic evaluation, when the probability of success with a percutaneous procedure is uncertain, or when the undesirable effects of a failed procedure are high. Logistic factors unique to each institution, such as surgical or interventional staff availability, may also play a role in the decision. Future research is needed to investigate the optimal timing and method of source control in patients with sepsis and septic shock with a source of infection amenable to drainage.

\section{Recommendation}

28. For adults with sepsis or septic shock, we recommend prompt removal of intravascular access devices that are a possible source of sepsis or septic shock after other vascular access has been established Best Practice Statement

\section{Rationale}

Removal of a potentially infected intravascular access device is considered a part of adequate source control [262]. An intravascular device suspected to be a source of sepsis should be removed after establishing another site for vascular access and following successful initial resuscitation $[265,266]$. In the absence of septic shock or fungemia, some implanted tunnelled catheter infections may be treated effectively with prolonged antimicrobial therapy if removal of the catheter is not practical [273]. However, catheter removal with adequate antimicrobial therapy is definitive and is the preferred treatment in most cases.

We identified one relevant RCT [274] and two observational studies $[275,276]$. There was no evidence of a difference in mortality, however, the studies were hampered by significant limitations, including risk of confounding by indication (the observational studies) and imprecision (the RCT), which is why the results should be interpreted cautiously. The quality of evidence was very low.

\section{De-escalation of antibiotics}

\section{Recommendation}

29. For adults with sepsis or septic shock, we suggest daily assessment for de-escalation of antimicrobials over using fixed durations of therapy without daily reassessment for de-escalation

Weak recommendation, very low quality of evidence

\section{Rationale}

Antimicrobial exposure is linked to the development of antimicrobial resistance and efforts to reduce both the 
number of antibiotics administered and their spectrum of therapy are therefore important strategies in patients with sepsis and septic shock [165]. This is particularly relevant in empiric therapy where broad-spectrum therapy is recommended, as the causative pathogen has not yet been identified. Once both the pathogen(s) and susceptibilities are known, antimicrobial de-escalation-i.e. stopping an antimicrobial that is no longer necessary (in case of combination therapy) or changing an antimicrobial to narrow the spectrum is encouraged. Given the adverse societal and individual risks to continued unnecessary antimicrobial therapy, thoughtful de-escalation of antimicrobials based on adequate clinical improvement is appropriate even if cultures are negative. Early discontinuation of all antimicrobial therapy if infection is ruled out is advisable [277]. Antimicrobial de-escalation should ideally be done as soon as possible, and rapid diagnostic techniques may facilitate this.

We identified direct evidence from 13 studies (1968 patients) [277], including 1 RCT [278]. In our metaanalysis, we observed improved short-term mortality in patients who were de-escalated (RR 0.72; 95\% CI 0.570.91) (Supplementary Appendix 2). Long-term mortality was evaluated in one study only and did not demonstrate a difference (RR 0.99; 95\% CI 0.64-1.52). De-escalation was associated with shorter length of stay in the hospital (MD -5.56 days; $95 \% \mathrm{CI}-7.68$ to -3.44 ), but not in the ICU (MD -2.6 days; $95 \% \mathrm{CI}-5.91$ to 0.72 ).

Most studies were observational, and there are concerns that de-escalation is used primarily in patients who are getting better, which is why the reported improved short-term mortality should be interpreted with caution $[277,279]$.

De-escalation is in generally safe, may offer cost savings when unnecessary antibiotics are discontinued, and reduced risk of antimicrobial resistance and reduced toxicity and side-effects may be important [280]. Based on the overall very low quality of evidence, RCTs are warranted along with more studies on antimicrobial resistance.

\section{Duration of antibiotics}

\section{Recommendation}

30. For adults with an initial diagnosis of sepsis or septic shock and adequate source control, we suggest using shorter over longer duration of antimicrobial therapy

Weak recommendation, very low quality of evidence

\section{Rationale}

Restricting antimicrobial therapy to the shortest course associated with better outcomes is an important part of antimicrobial stewardship [281-285]. The optimal duration of antimicrobial therapy for a given patient with sepsis or septic shock depends on many factors, including host, microbe, drug, and anatomical site (Table 2) $[99,100]$.

There have been considerable efforts over the past two decades to clarify the optimal duration of antimicrobial therapy by comparing "short" courses with traditional ("longer") courses. There are data from RCTs in specific conditions such as pneumonia [286-289], urinary tract infections [290], bacteremia [291, 292], and intraabdominal infections [293]. In many of the trials, the shorter course was just as effective as the longer course but associated with fewer adverse consequences. Very few trials, however, focussed exclusively on critically ill patients with sepsis or septic shock, and the overall quality of evidence was very low.

Given the lack of definitive and generalizable data regarding the optimal duration of therapy for patients who are critically ill, it is not surprising that there is considerably practice variation [281, 294]. Specialist consultation appears to be associated with improved patient outcomes for a variety of infectious syndromes [295300]. This has generally been ascribed to improvements in microbial appropriateness of the empiric antimicrobial regimen provided. However, it is also possible that reducing the duration of unnecessary therapy may account for at least part of the benefit.

Thus, for adults with an initial diagnosis of sepsis or septic shock and adequate source control, we suggest a shorter course of antibiotics, as this is less costly, has fewer undesirable effects without impacting adversely on outcomes (see Table 4).

\section{Biomarkers to discontinue antibiotics}

\section{Recommendation}

31. For adults with an initial diagnosis of sepsis or septic shock and adequate source control where optimal duration of therapy is unclear,

we suggest using procalcitonin AND clinical evaluation to decide when to discontinue antimicrobials over clinical evaluation alone

Weak recommendation, low quality of evidence

\section{Rationale}

Shorter durations of antimicrobial therapy are in general recommended; however, critically ill patients often receive antimicrobials for more days than necessary [288, 301, 306]. While typically clinical evaluation alone is used to decide duration, biomarkers could 
Table 2 Examples of risk factors for fungal infection

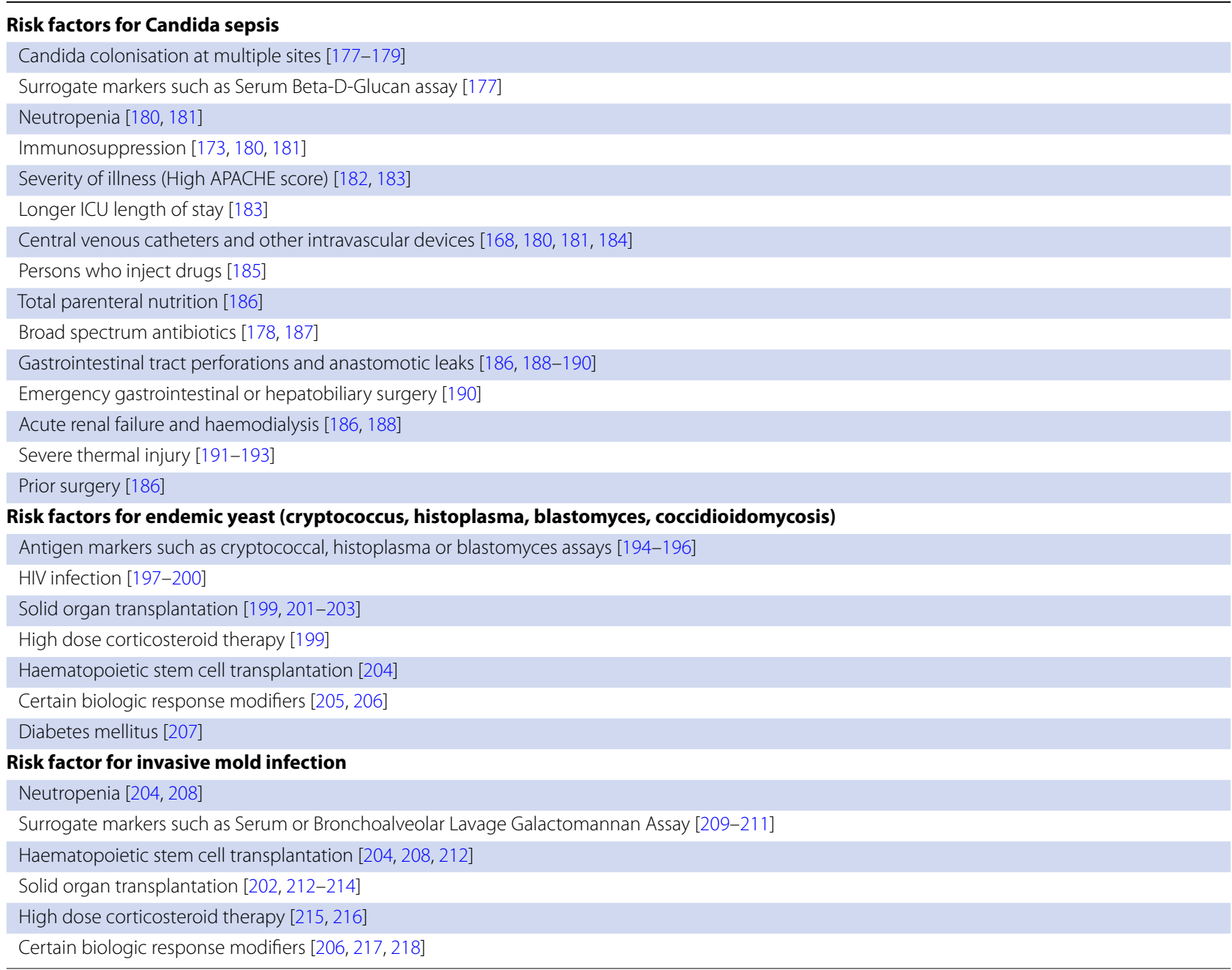

The decision to start empirical antifungal therapy depends on the type and number of risk factors, along with the locale epidemiology of fungal infections

offer additional information. C Reactive Protein is often used in this regard. Procalcitonin has been studied most extensively both in critically ill and non-critically ill patients, both for initiation and discontinuation of therapy [307].

We identified direct evidence from 14 RCTs $(n=4499$ patients) that assessed use of procalcitonin to guide antimicrobial treatment duration in patients with sepsis (two trials included critically ill patients in general) [308-321]. A meta-analysis suggested improved mortality in patients who were managed using procalcitonin versus control (RR 0.89; 95\% CI 0.80-0.99), while there was no effect on length of stay in ICU or hospital.
Antibiotic exposure was consistently lower in patients who were managed with procalcitonin and clinical evaluation, however, in many trials the total duration of therapy was still 7 days or longer in the intervention group. Also, the algorithms for antimicrobial therapy, frequency of procalcitonin monitoring and the thresholds (or percentage change in procalcitonin concentration) for discontinuation differed across the trials. Therefore, the overall quality of evidence was judged to be low.

The undesirable effects of using procalcitonin along with clinical evaluation to decide when to discontinue antimicrobials are considered minimal, and do not 
Table 3 Guidance for PK/PD-based dosing for specific drug classes

\begin{tabular}{|c|c|c|c|}
\hline Drug or drug class & $\begin{array}{l}\text { PK/PD index associated } \\
\text { with bacterial killing or } \\
\text { efficacy }\end{array}$ & Drug concentration target & Considerations for optimised dosing ${ }^{a}$ \\
\hline
\end{tabular}

\section{Antibacterials}

\begin{tabular}{|c|c|c|c|c|}
\hline Aminoglycosides & $\mathrm{AUC}_{0-24} / \mathrm{MIC} ; \mathrm{C}_{\max } / \mathrm{MIC}$ & $\begin{array}{l}\text { AUC } 70-100 \\
C_{\max } / \mathrm{MIC} 8-10\end{array}$ & $\begin{array}{l}\text { Use extended interval dosing with patient } \\
\text { weight and kidney function }\end{array}$ & [237] \\
\hline Beta-lactams & $f \mathrm{~T}_{>\mathrm{MIC}}$ & $\mathrm{C}_{\min }>\mathrm{MIC}$ & $\begin{array}{l}\text { Use prolonged infusions, consider patient } \\
\text { weight and kidney function }\end{array}$ & [253] \\
\hline Colistin & $\mathrm{AUC}_{0-24} / \mathrm{MIC}$ & Unspecified & Use patient weight and kidney function & [259] \\
\hline Daptomycin & $\mathrm{AUC}_{0-24} / \mathrm{MIC} ; \mathrm{C}_{\max } / \mathrm{MIC}$ & $\mathrm{AUC}_{0-24} / \mathrm{MIC}>200$ & Use patient weight and kidney function & [237] \\
\hline Fluoroquinolones & $\mathrm{AUC}_{0-24} / \mathrm{MIC} ; \mathrm{C}_{\max } / \mathrm{MIC}$ & $\mathrm{AUC}_{0-24} / \mathrm{MIC} 80-125$ & Use kidney function & [237] \\
\hline Vancomycin & $\mathrm{AUC}_{0-24} / \mathrm{MIC}$ & $\mathrm{AUC}_{0-24} / \mathrm{MIC} 400$ & Use patient weight and kidney function & [260] \\
\hline \multicolumn{5}{|l|}{ Antifungals } \\
\hline Fluconazole & $\mathrm{AUC}_{0-24} / \mathrm{MIC}$ & $\mathrm{AUC}_{0-24} / \mathrm{MIC} 100$ & Use patient weight and kidney function & {$[261]$} \\
\hline Posaconazole & $\mathrm{AUC}_{0-24} / \mathrm{MIC}$ & $C_{\min } 1-4 \mathrm{mg} / \mathrm{L}$ & Use formulation-specific dose & [261] \\
\hline Voriconazole & $\mathrm{AUC}_{0-24} / \mathrm{MIC}$ & $C_{\min } 2-6 \mathrm{mg} / \mathrm{L}$ & Use patient weight & [261] \\
\hline
\end{tabular}

$A U C_{0-24}$ ratio of area under the concentration-time curve from 0 to $24 \mathrm{~h}, M I C$ minimum inhibitory concentration, $f T_{>M I C}$ time overdosing interval that free (unbound) drug is maintained above the $\mathrm{MIC}, C_{\max }$ maximum concentration in a dosing interval, $C_{\min }$ minimum concentration in a dosing interval

a Other considerations than those listed may have been listed in studies in critically ill patient sub-populations

outweigh the potential benefits [322]. Limited data on the cost-effectiveness are available, although a single centre study reported decreased hospital costs associated with PCT-guided antibiotic in medical ICU patient with undifferentiated sepsis [323]. Procalcitonin testing may not be available in all countries and healthcare settings, including LMICs.
Based on apparent benefit and no obvious undesirable effects, we suggest using procalcitonin along with clinical evaluation to decide when to discontinue antimicrobials in adults with an initial diagnosis of sepsis or septic shock and adequate source control, if the optimal duration of therapy is unclear and if procalcitonin is available.

Table 4 Planned duration of empirical antimicrobial therapy in RCTs of shorter versus longer duration of therapy according to clinical syndrome

\begin{tabular}{|c|c|c|c|c|c|}
\hline \multirow{3}{*}{$\begin{array}{l}\text { Population/syndrome } \\
\text { Pneumonia }\end{array}$} & \multicolumn{2}{|c|}{$\mathrm{RCT} /$ systematic review (data extracted from) } & \multirow{2}{*}{$\begin{array}{l}\text { Shorter duration } \\
8 \text { days }\end{array}$} & \multirow{2}{*}{$\begin{array}{l}\text { Longer duration } \\
15 \text { days }\end{array}$} & \multirow{2}{*}{$\begin{array}{l}\text { Outcomes } \\
\text { No difference }\end{array}$} \\
\hline & [301] & Capellier (2012) & & & \\
\hline & {$[301,302]$} & Chastre (2003) & 8 days & 15 days & No difference \\
\hline & [302] & El Moussaoui (2006) & 3 days & 8 days & No difference \\
\hline & [301-303] & Fekih Hassen (2009) & 7 days & 10 days & No difference \\
\hline & {$[302,303]$} & File (2007) & 5 days & 7 days & No difference \\
\hline & {$[302,303]$} & Kollef (2012) & 7 days & 10 days & No difference \\
\hline & {$[302,303]$} & Leophonte (2002) & 5 days & 10 days & No difference \\
\hline & [301] & Medina (2007) & 8 days & 12 days & No difference \\
\hline & {$[302,303]$} & Siegel (1999) & 7 days & 10 days & No difference \\
\hline & {$[302,303]$} & Tellier (2004) & 5 days & 7 days & No difference \\
\hline \multirow[t]{3}{*}{ Bacteremia } & [302] & Chaudhry (2000) & 5 days & 10 days & No difference \\
\hline & [302] & Runyon (1991) & 5 days & 10 days & No difference \\
\hline & [304] & Yahav (2018) & 7 days & 14 days & No difference \\
\hline \multirow[t]{2}{*}{ Intra-abdominal infection } & [305] & Montravers (2018) & 8 days & 15 days & No difference \\
\hline & [293] & Sawyer (2015) & Max. 5 days & Max. 10 days & No difference \\
\hline Urinary tract infection & [290] & Peterson (2008) & 5 days & 10 days & No difference \\
\hline
\end{tabular}




\section{Haemodynamic management Fluid management}

Recommendations
32. For adults with sepsis or septic shock, we recommend using crystal-
loids as first-line fluid for resuscitation
Strong recommendation, moderate quality of evidence
33. For adults with sepsis or septic shock, we suggest using balanced
crystalloids instead of normal saline for resuscitation
Weak recommendation, low quality of evidence
34. For adults with sepsis or septic shock, we suggest using albumin in
patients who received large volumes of crystalloids over using crystal-
loids alone
Weak recommendation, moderate quality of evidence
35. For adults with sepsis or septic shock, we recommend against using
starches for resuscitation
Strong recommendation, high quality of evidence
36. For adults with sepsis and septic shock, we suggest against using
gelatin for resuscitation
Weak recommendation, moderate quality

\section{Rationale}

Fluid therapy is a key part of the resuscitation of sepsis and septic shock. Crystalloids have the advantage of being inexpensive and widely available. The absence of clear benefit following the administration of colloids compared to crystalloid solutions supports the use of crystalloid solutions in the resuscitation of patients with sepsis and septic shock [324]. The optimal fluid remains a subject of debate. For decades, the administration of normal saline solution $(0.9 \%$ sodium chloride) has been common practice [325], but potential adverse effects that include hyperchloremic metabolic acidosis, renal vasoconstriction, increased cytokine secretion and concern about acute kidney injury (AKI) have led to increased interest in chloride-restrictive solutions, known as balanced or buffered solutions [326-330]. Subsequently, a network meta-analysis of 14 RCTs of patients with sepsis showed in an indirect comparison that balanced crystalloids were associated with decreased mortality, compared to saline [331].

There have been a number of recent RCTs assessing the question of which crystalloid may be most beneficial in patients with sepsis. In the SPLIT multicentre, doubleblinded clinical trial, the comparison between balanced solutions and normal saline yielded no differences in mortality or AKI [332]. The modest volume of infused fluid, the predominance of surgical patients, and the low number of septic patients (4\%) precludes generalizability of the results. In 2016 , the SALT pilot trial $(n=974)$ compared balanced solutions versus normal saline; with septic patients comprising $25 \%$ and $28 \%$ of the population, respectively [333]. The primary outcome, a composite outcome including mortality, new RRT or persistent renal dysfunction (major adverse kidney event within
30 days, MAKE30), was similar between groups $(24.6 \%$ vs. $24.7 \%$ ). Subsequently, the SMART trial was published in 2018, a single-centre, multiple-crossover study including 15,802 patients who received balanced solutions or normal saline, alternating on a monthly basis [334]. In the pre-specified subgroup of patients admitted with sepsis in all participating ICUs, 30-day mortality was lower in those receiving balanced solutions, compared to normal saline (OR 0.80; 95\% CI 0.67-0.94). Likewise, in a secondary analysis including only the 1,641 patients admitted to medical ICUs with a diagnosis of sepsis, balanced solutions were associated with reduced 30-day hospital mortality (OR 0.74; 95\% CI 0.59-0.93) and MAKE30, and increased vasopressor- and RRT-free days [335].

The SMART trial was a single-centre study without individual patient randomisation and no blinded assignment of the intervention, it exposed participants to moderate amount of fluid volume, identification of sepsis subgroups was based on ICD-10 codes, and it used a composite outcome which may not be as relevant as a patient-centered outcome [336]. However, the use of balanced solutions in sepsis may be associated with improved outcomes compared with chloride-rich solutions. No cost-effectiveness studies compared balanced and unbalanced crystalloid solutions. Therefore, we considered the desirable and undesirable consequences to favour balanced solutions, but as the quality of the evidence is low, we issued a weak recommendation. Two ongoing large RCTs will provide additional data and inform future guideline updates [337, 338].

Although albumin is theoretically more likely to maintain oncotic pressure than crystalloids [339], it is more costly and there is no clear benefit with its routine use. Since the publication of the 2016 guidelines [12] two single-centre trials and two meta-analyses have been published on this topic [324, 340-342]. A Cochrane review including RCTs with 12,492 patients comparing albumin versus crystalloids found no difference in 30-day (RR 0.98 ; 95\% CI $0.92-1.04$ ) or 90-day mortality (RR 0.98; 95\% CI 0.92-1.04) or need for RRT between groups (RR 1.11; 95\% CI 0.96-1.27) [324]. This metaanalysis included patients with critical illness, and while the main solution included in the analysis was albumin, some studies in other analyses included fresh frozen plasma. A second meta-analysis, which also included critically ill patients, found lower static filling pressures ( $\left.\mathrm{MD}-2.3 \mathrm{~cm} \mathrm{H}_{2} \mathrm{O} ; 95 \% \mathrm{CI} 3.02-1.05\right)$ and mean arterial pressure (MAP) (MD-3.53 $\mathrm{mmHg}$; 95\% CI-6.71 to -0.36) with crystalloid use, but no difference in mortality at 28 days (RR 1.0 ; $95 \%$ CI $0.92-1.10$ ) or 90 days (RR 1.32; 95\% CI 0.76-2.29) [340]. The largest clinical trial in sepsis, the ALBIOS trial comparing a combination of albumin and crystalloids to crystalloids alone in 
1818 patients with sepsis or septic shock did not demonstrate a difference in 28-day (RR 1.0; 95\% CI 0.87-1.14) or 90-day mortality (RR 0.94; 95\% CI $0.85-1.05$ ) [339]. Of note, in this trial, albumin was given as a $20 \%$ solution, with a treatment goal of a serum albumin concentration of $30 \mathrm{~g} / \mathrm{L}$ until ICU discharge or 28 days. A meta-analysis of studies including septic patients did not show a significant difference in mortality (RR 0.98; 95\% CI 0.89-1.08). In addition, the risk of new organ failures (RR 1.02; 95\% CI $0.93-1.11$ ), ventilator-free days or vasopressor-free days did not differ. Although albumin use resulted in a larger treatment effect in the septic shock subgroup (RR 0.88 ; 95\% CI 0.77-0.99) than in the sepsis subgroup (RR 1.03; 95\% CI 0.91-1.17), the subgroup analysis did not detect a subgroup effect $(P$-interaction $=0.19)$.

The lack of proven benefit and higher cost of albumin compared to crystalloids contributed to our strong recommendation for the use of crystalloids as first-line fluid for resuscitation in sepsis and septic shock. The suggestion to consider albumin in patients who received large volumes of crystalloids is informed by evidence showing higher blood pressure at early and later time points [339], higher static filling pressures [340], and lower net fluid balance [339] with albumin. Limited data precludes a cutoff value for crystalloid infusion above which albumin might be considered as part of resuscitation.

In the 2016 SSC guidelines, a strong recommendation was issued against using hydroxyethyl starch (HES) [12]. No new data were identified. A previous meta-analysis of RCTs in septic patients showed a higher risk of RRT with the use of HES 130/0.38-0.45 (RR 1.36; 95\% CI 1.081.72) and a higher risk of death in a pre-defined analysis of low risk of bias trials (RR 1.11; 95\% CI 1.0-1.2) [343]. A network meta-analysis of patients with sepsis or septic shock also demonstrated a higher risk of death (OR 1.1; 95\% CI 0.99-1.30) and need for RRT (OR 1.39; 95\% CI 1.17-1.66) [331] with starches in a direct comparison with crystalloids. Therefore, the 2016 recommendation against the use of HES in resuscitation of patients with sepsis or septic shock did not change [331, 343].

Gelatin is a synthetic colloid used as a resuscitation fluid; there is a lack of powered well-designed studies supporting its administration in sepsis and septic shock. Included studies are generally small and include mostly post-operative, non-critically ill patients. In an indirect comparison, a 4-node network meta-analysis conducted in patients with sepsis, showed no clear effect on mortality when compared to crystalloids (OR 1.24; $95 \%$ credible interval [CrI] 0.61-2.55) [331]. Similarly, another RCT did not find an effect on mortality with gelatin use (RR 0.87; 95\% CI 0.66-1.12) [344]. Adverse effects of gelatin have been reviewed in a network meta-analysis, which demonstrated higher risk of RRT with gelatin use compared to normal saline (OR 1.27; 95\% CrI 0.44-3.64) and balanced crystalloids (OR 1.50; 95\% CrI 0.56-3.96) [345]. Overall, the quality of evidence was moderate, due to imprecision and indirectness. In a systematic review of RCTs including patients with hypovolemia, gelatin use increased the risk of anaphylaxis (RR 3.01; 95\% CI 1.27-7.14) in comparison with crystalloids use [346]. Furthermore, gelatins may affect haemostasis and the effect on blood transfusions was unclear (RR 1.10; 95\% CI 0.86-1.41). Therefore, in the face of inconclusive effect on mortality, increased adverse effects, and higher costs, the panel issued a weak recommendation against the use of gelatin for acute resuscitation. More high-quality studies are needed to inform future guideline updates.

\section{Vasoactive agents}

Recommendations

37. For adults with septic shock, we recommend using norepinephrine as the first-line agent over other vasopressors. Strong recommendation Dopamine. High quality evidence

Vasopressin. Moderate-quality evidence

Epinephrine. Low-quality evidence

Selepressin. Low-quality evidence

Angiotensin II. Very low-quality evidence

\section{Remark}

In settings where norepinephrine is not available, epinephrine or dopamine can be used as an alternative, but we encourage efforts to improve the availability of norepinephrine. Special attention should be given to patients at risk for arrhythmias when using dopamine and epinephrine

38. For adults with septic shock on norepinephrine with inadequate MAP levels, we suggest adding vasopressin instead of escalating the dose of norepinephrine

Weak recommendation, moderate-quality evidence

Remark

In our practice, vasopressin is usually started when the dose of norepinephrine is in the range of $0.25-0.5 \mu \mathrm{g} / \mathrm{kg} / \mathrm{min}$

39. For adults with septic shock and inadequate MAP levels despite norepinephrine and vasopressin, we suggest adding epinephrine

Weak recommendation, low-quality evidence

40. For adults with septic shock, we suggest against using terlipressin Weak recommendation, low quality of evidence

\section{Rationale}

Norepinephrine is a potent $\alpha-1$ and $\beta-1$ adrenergic receptors agonist, which results in vasoconstriction and increased MAP with minimal effect on heart rate. Dopamine acts in a dose-dependent fashion on dopamine-1, $\alpha-1$ and $\beta-1$ adrenergic receptors. At lower dosages, dopamine causes vasodilation via dopamine-1 receptor activity in the renal, splanchnic, cerebral, and coronary beds. With higher dosages, dopamine's $\alpha$-adrenergic receptor activity predominates resulting in vasoconstriction and increased systemic vascular resistance (SVR); its $\beta-1$ adrenergic receptor activity can lead to dose-limiting arrhythmias. Norepinephrine is more potent than 


\section{Vasoactive Agent Management}

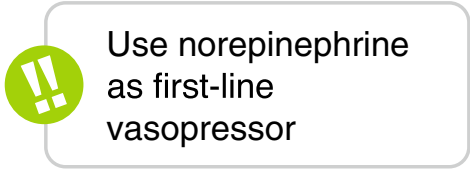

For patients with septic shock on vasopressor

Target a MAP of $65 \mathrm{~mm} \mathrm{Hg}$

Consider invasive
monitoring of arterial
blood pressure

If central access is not yet available

Consider initiating
vasopressors
peripherally*

If MAP is inadequate despite low-to-moderate-dose norepinephrine

Consider adding vasopressin
If cardiac dysfunction with persistent hypoperfusion is present despite adequate volume status and blood pressure

Consider adding dobutamine or switching to epinephrine

(1) Strong recommendations (1) Weak recommendations

*When using vasopressors peripherally, they should be administered only for a short period of time and in a vein proximal to the antecubital fossa.

Fig. 2 Summary of vasoactive agents recommendations

dopamine as a vasoconstrictor. In a systematic review and meta-analysis of 11 RCTs, norepinephrine resulted in a lower mortality (RR $0.89 ; 95 \%$ CI $0.81-0.98$ ) and lower risk of arrhythmias (RR 0.48; 95\% CI 0.40-0.58) compared with dopamine [347]. Although the $\beta-1$ activity of dopamine may be useful in patients with myocardial dysfunction, the higher risk of arrhythmias limits its use [348].

Epinephrine's action is also dose-dependent with potent $\beta-1$ adrenergic receptor activity and moderate $\beta-2$ and $\alpha-1$ adrenergic receptor activity. The activity of epinephrine, at low doses, is primarily driven by its action on $\beta-1$ adrenergic receptors, resulting in increased cardiac output $(\mathrm{CO})$, decreased systemic vascular resistance (SVR) and variable effects on MAP. At higher doses, however, epinephrine administration results in increased SVR and CO. Potential adverse effects of epinephrine include arrhythmias and impaired splanchnic circulation [349]. Epinephrine may increase aerobic lactate production via stimulation of skeletal muscle $\beta-2$ adrenergic receptors, making the use of serum lactate to guide resuscitation challenging [350]. A randomised blinded study comparing epinephrine with norepinephrine in patients with shock showed no difference in 90-day mortality (HR 0.88; 95\% CI 0.63-1.25) and vasopressor-free days [351]. The panel issued a strong recommendation for norepinephrine as the first-line agent over other vasopressors (Fig. 2).

Vasopressin is an endogenous peptide hormone produced in the hypothalamus and stored and released by the posterior pituitary gland. Its mechanism for vasoconstrictive activity is multifactorial and includes binding of $\mathrm{V}_{1}$ receptors on vascular smooth muscle resulting in increased arterial blood pressure. Studies show that vasopressin concentration is elevated in early septic shock but decreases to normal range in the majority of patients between 24 and $48 \mathrm{~h}$ as shock continues [352, 353]. This finding has been called "relative vasopressin deficiency" as, in the presence of hypotension, vasopressin would be expected to be elevated. The significance of this finding is unknown. Unlike most vasopressors, vasopressin is not titrated to response, but it is usually administered at a fixed dose of 0.03 units $/ \mathrm{min}$ for the treatment of septic shock. In clinical trials, vasopressin was used up to 0.06 units/min [354]. Higher doses of vasopressin have been associated with cardiac, digital, and splanchnic ischaemia [355].

The VANISH trial directly compared the use of vasopressin versus norepinephrine by randomizing patients with septic shock in a factorial $2 \times 2$ design aiming to also assess the role of hydrocortisone. There was no significant difference between the vasopressin and norepinephrine groups in 28 -day mortality [ $30.9 \%$ vs $27.5 \%$; RR 
1.13 (95\% CI 0.85-1.51). Although there was no difference with respect to kidney injury (RR 0.89 ; 95\% CI 0.721.11), vasopressin use reduced the risk of RRT (RR 0.71; 95\% CI 0.53-0.97) [354].

As for combination therapy, the main study (the VASST trial) comparing norepinephrine alone to norepinephrine plus vasopressin $(0.01-0.03 \mathrm{U} / \mathrm{min})$ showed no improvement in 28 -day mortality $(39.3 \%$ vs $35.4 \%, p=0.26)$ [356]. However, in a subgroup analysis, patients with less severe shock receiving norepinephrine $<15 \mu \mathrm{g} / \mathrm{min}$ had improved survival with the addition of vasopressin $(26.5 \%$ vs. $35.7 \%, p=0.05)$. Both VANISH and VASST demonstrated a catecholamine-sparing effect of vasopressin; as such, the early use of vasopressin in combination with norepinephrine may help reduce the adrenergic burden associated with traditional vasoactive agents [357]. In our systematic review of 10 RCTs, vasopressin with norepinephrine reduced mortality as compared to norepinephrine alone (RR 0.91; 95\% CI 0.83-0.99) but did not reduce the need for RRT (RR 0.79; 95\% CI 0.57-1.10). There was no difference in the risks of digital ischaemia (RR 1.01; 95\% CI 0.33-9.84) or arrhythmias (RR 0.88; 95\% CI $0.63-1.23)$. The threshold for adding vasopressin varied among studies and remains unclear. Starting vasopressin when norepinephrine dose is in the range of $0.25-0.5 \mu \mathrm{g} /$ $\mathrm{kg} / \mathrm{min}$ seems sensible [354]. Another meta-analysis of RCTs on distributive shock showed a lower risk of atrial fibrillation with the combination of vasopressin and norepinephrine compared to norepinephrine alone [358]. However, a recent individual patient data meta-analysis of patients with septic shock from 4 RCTs showed that vasopressin alone or in combination with norepinephrine led to higher risk of digital ischaemia (risk difference [RD] 1.7\%; 95\% CI 0.3-3.2) but lower risk of arrhythmia (RD-2.8\%; 95\% CI-0.2 to-5.3) compared to norepinephrine alone [359].

The evidence regarding the optimal therapeutic strategy for shock requiring high dose vasopressors is scant [360]. Epinephrine has been suggested as second or thirdline vasopressor for patients with septic shock. With the use of norepinephrine at elevated concentrations, the $\alpha 1$ receptors may already be saturated and downregulated [361]. Thus, the use of another drug such as epinephrine that targets the same receptors may be of limited utility and vasopressin could be more adequate in this scenario. In an indirect comparison, a network meta-analysis did not find any significant difference between epinephrine and vasopressin in terms of mortality (RR 0.94; $95 \% \mathrm{CI}$ $0.47-1.88$ ) [362]. Epinephrine might be useful in refractory septic shock patients with myocardial dysfunction.
Thus, we considered the desirable and undesirable consequences of these vasopressors and issued a strong recommendation to use norepinephrine as a first line agent instead of dopamine, vasopressin, epinephrine and selepressin and angiotensin II in patients with septic shock as a first-line agent, and a weak recommendation over selepressin and angiotensin II. Although some evidence suggests that vasopressin might be superior to norepinephrine in terms of clinical outcomes, the panel took into consideration its higher costs and lower availability and have issued a strong recommendation to use norepinephrine as first line agent instead of vasopressin. We also consider the potential benefit and undesirable consequences of using the combination of norepinephrine and vasopressin and issue a weak recommendation for adding vasopressin instead of escalating the dose of norepinephrine. Further evidence is needed to properly address the role of combination therapy of vasopressors in septic shock.

The panel also recognised that availability of, and experience with, norepinephrine may vary. As part of the global campaign for universal healthcare, the World Health Organisation (WHO) essential medicines and health products programme works to increase global access to essential, high-quality, safe, effective, and affordable medical products. If norepinephrine is unavailable, either dopamine or epinephrine can be used with special attention given to the risk of arrhythmias.

Selepressin is a highly selective V1 agonist, inducing vasoconstriction via stimulation of vascular smooth muscle. It does not share the typical V1b and V2 receptor effects of vasopressin (increased pro-coagulant factors, salt, and water retention, nitric oxide, and corticosteroid release) and has, therefore, been postulated as a potentially attractive non-catecholamine vasopressor alternative to norepinephrine. Selepressin has been studied in two randomised trials in septic shock. The first, a double-blind, randomised, placebo-controlled phase IIa trial, compared three ascending doses of selepressin (1.25, 2.5 and $3.75 \mathrm{ng} / \mathrm{kg} / \mathrm{min}$ ) in maintaining blood pressure, with open-label norepinephrine [363]. Selepressin at a dose of $2.5 \mathrm{ng} / \mathrm{kg} / \mathrm{min}$ was demonstrated to be effective in maintaining MAP $>60 \mathrm{mmHg}$ without norepinephrine in about $50 \%$ of patients at $12 \mathrm{~h}$ and about $70 \%$ of patients at $24 \mathrm{~h}$. A follow-on phase IIb/phase III trial using an adaptive design, initially comparing three doses $(1.7,2.5$ and $3.5 \mathrm{ng} / \mathrm{kg} / \mathrm{min}$ ) with the potential to add a further $5 \mathrm{ng} /$ $\mathrm{kg} / \mathrm{min}$ dose group [364]. The study was stopped for futility after enrolment of 828 patients, with no significant differences between any of the key endpoints [ventilator- and vasopressor-free days, 15.0 (selepressin) versus 
14.5 (placebo), $p=0.30$; 90-day all-cause mortality, $40.6 \%$ vs $39.4 \%, p=0.77$; 30 -day RRT-free days, 18.5 vs 18.2 , $p=0.85$; 30-day ICU-free days, 12.6 vs $12.2, p=0.41$; adverse event rates were also similar between groups. The meta-analysis of the two studies did not show significant difference in mortality [selepressin: $41.8 \%$ vs norepinephrine: 40.45\%; RR 0.99 (95\% CI 0.84-1.18)]. As selepressin failed to demonstrate clinical superiority over norepinephrine, we considered the desirable and undesirable consequences to be in favour of norepinephrine and issued a weak recommendation against the use of selepressin as a first-line therapy. Furthermore, it is not currently commercially available.

Angiotensin II is a naturally occurring hormone with marked vasoconstrictor effects, triggered through stimulation of the renin-angiotensin system. A synthetic human preparation has recently become available for clinical use and has been studied in two clinical trials. After a small, short-term pilot of 20 patients with vasodilatory (septic) shock 10 patients in each group which showed physiological efficacy without obvious safety issues [365], a larger RCT of 344 patients was performed in patients with vasodilatory shock (approximately 90\% confirmed or presumed sepsis) [366]. The primary endpoint, an increase of MAP of at least $10 \mathrm{mmHg}$ or to at least $75 \mathrm{mmHg}$, was achieved in 114 of 163 patients in the angiotensin II group and in 37 of 158 patients in the placebo group $(69.9 \%$ vs $23.4 \%$, $p<0.001)$. A meta-analysis found no difference in mortality rates between angiotensin II and norepinephrine (46.2\% vs $54.2 \%$; RR 0.85 (95\% CI $0.69-1.06$ ); very low quality). There was no clear increase in adverse events with the use of angiotensin II. As the available evidence is of very low quality, and clinical experience in sepsis and, therefore, demonstration of safety remains limited, the panel considered that angiotensin should not be used as a first-line agent, but having demonstrated physiological effectiveness, it may have a role as an adjunctive vasopressor therapy.

Terlipressin is a prodrug and is converted to lysine vasopressin by endothelial peptidases, producing a "slow release" effect and giving an effective half-life of around $6 \mathrm{~h}$. Terlipressin is more specific for the V1 receptors and it has been studied in 9 clinical trials of patients with sepsis, with or without cirrhosis, involving 950 patients in total. Our meta-analysis showed no difference in mortality (terlipressin: 42.9\% vs 49.0\%; RR 0.89 (95\% CI 0.70-1.13); low quality) but an increase in adverse events. The largest of these studies enrolled 617 patients with septic shock, in a randomised, blinded fashion, with terlipressin (or placebo) added at a dose of between 20 to $160 \mathrm{mcg} / \mathrm{h}$ to a standard norepinephrine-based approach, to achieve a MAP of $65-75 \mathrm{mmHg}$ [367]. The primary outcome was death from any cause at 28 days. The 28 -day mortality in the two groups was $40 \%$ for terlipressin and 38\% for norepinephrine (OR 0.93; 95\% CI 0.55-1.56, $p=0.80$ ), and there were no differences in SOFA score at day 7 or vasopressor free days. More patients who received terlipressin had serious adverse events; 33 of 260 (12\%) patients experienced digital ischaemia after receiving terlipressin, versus only one patient who received norepinephrine $(p<0.0001)$; diarrhea was also more common in the terlipressin group ( $2.7 \%$ versus $0.35 \%, p=0.037)$. There were three cases of mesenteric ischaemia in the terlipressin group versus one in the norepinephrine group. Therefore, the panel considered that the undesirable consequences are higher with the use of terlipressin and issued a weak recommendation against its use in patients with septic shock.

\section{Inotropes \\ Recommendations}

41. For adults with septic shock and cardiac dysfunction with persistent hypoperfusion despite adequate volume status and arterial blood pressure, we suggest either adding dobutamine to norepinephrine or using epinephrine alone

Weak recommendation, low quality of evidence

42. For adults with septic shock and cardiac dysfunction with persistent hypoperfusion despite adequate volume status and arterial blood pressure, we suggest against using levosimendan

Weak recommendation, low quality of evidence

\section{Rationale}

Sepsis-induced myocardial dysfunction is recognised as a major contributor to the haemodynamic instability and is associated with worse outcomes of patients with septic shock [368]. Inotropic therapy can be used in patients with persistent hypoperfusion after adequate fluid resuscitation, and in patients with myocardial dysfunction, based on suspected or measured low $\mathrm{CO}$ and elevated cardiac filling pressures. Dobutamine and epinephrine are the most commonly used inotropes. Physiologic studies demonstrate that dobutamine increases $\mathrm{CO}$ and oxygen transport, increases splanchnic perfusion and tissue oxygenation, improves intramucosal acidosis and hyperlactatemia [369]. However, these effects may not be predictable [370]. Dobutamine infusion may produce severe vasodilation and result in lower MAP. In addition, the inotropic response may be blunted in sepsis with a preserved chronotropic effect causing tachycardia without an increase in stroke volume (SV) [370]. No 
RCTs compared dobutamine to placebo in this population. Indirect comparison from network meta-analysis showed that dobutamine with norepinephrine had no clear impact on mortality when compared to no inotropic agents (OR 0.69; 95\% CI 0.32-1.47) [362]. None of the trials directly compared dobutamine combined with norepinephrine to norepinephrine alone. In an observational study of 420 patients with septic shock, the use of an inotropic agent (dobutamine, levosimendan, epinephrine, or milrinone) was independently associated with increased 90-day mortality (OR 2.29; 95\% CI 1.33-3.94) even after propensity score adjustment [371]. However, the analysis adjusted only to baseline characteristics, without accounting for time-varying confounders including the patient condition at the time of initiating inotropes which may explain the association with mortality. The panel considered the network meta-analysis as a higher quality than observational studies and issued a suggestion to use inotropes only in selected situations.

No evidence supports the superiority of dobutamine over epinephrine. Epinephrine is commonly available especially in low-resource settings [372]. In an indirect comparison of dobutamine versus epinephrine, a network meta-analysis showed no clear effect on mortality (OR 1.18; 95\% CI 0.47-3.97) [362]. Therefore, we considered the desirable and undesirable consequences to be comparable for both drugs and issued a weak recommendation to use either one for patients with septic shock and cardiac dysfunction with persistent hypoperfusion despite adequate fluid status and MAP. Both should be discontinued in the absence of improvement in hypoperfusion or in the presence of adverse events. Further evidence derived from high quality RCTs is needed to properly address the role of inotropes in sepsis.

Levosimendan is a calcium-sensitizing drug with inotropic and vasodilatory properties. It has been evaluated in septic shock [373]. A meta-analysis of three RCTs $(n=781)$ showed that levosimendan, compared to no inotropic agents, did not impact mortality (RR 0.87; 95\% CI $0.59-1.28)$. Data from the LeoPARDS trial $(n=515)$ showed that levosimendan versus no inotropic agents was associated with a lower likelihood of successful weaning from mechanical ventilation and a higher risk of supraventricular tachyarrythmia [373]. A meta-analysis of seven RCTs comparing levosimendan with dobutamine showed that levosimendan was not superior to dobutamine in adults with sepsis in terms of mortality (OR 0.80; 95\% CI 0.48, 1.33; $p=0.39$ ) [374]. Thus, the panel issued a weak recommendation against the use of levosimendan based on the lack of benefit, in addition to the safety profile, cost and the limited availability of the drug.

\section{Monitoring and intravenous access}

\section{Recommendations}

43. For adults with septic shock, we suggest using invasive monitoring of arterial blood pressure over non-invasive monitoring, as soon as practical and if resources are available

Weak recommendation, very low quality of evidence

44. For adults with septic shock, we suggest starting vasopressors peripherally to restore MAP rather than delaying initiation until a central venous access is secured

Weak recommendation, very low quality of evidence

Remark

When using vasopressors peripherally, they should be administered only for a short period of time and in a vein in or proximal to the antecubital fossa

\section{Rationale}

Estimation of blood pressure using a non-invasive cuff tends to be inaccurate and the discrepancy more pronounced in shock states [375-379]. Insertion of an arterial catheter permits safe, reliable and continuous measurement of arterial pressure and allows real time analysis so that therapeutic decisions can be based on immediate and accurate blood pressure information [380]. A systematic review of observational studies showed that the risk of limb ischaemia and bleeding was less than $1 \%$ for radial catheters, and the risk of limb ischaemia and bleeding was less than $1 \%$ and $1.58 \%$, respectively, for femoral catheters. The most common complication was localised haematoma, $14 \%$ for radial and $6 \%$ for femoral catheters [381]. Ultrasound guidance may increase the first attempt success rate and decrease the complication rate [382, 383]. A systematic review showed higher risk of infections when femoral arterial catheters were used compared to radial artery catheters (RR 1.93; 95\% CI 1.32-2.84), and the overall pooled incidence of bloodstream infection was 0.96 per 1000 catheter days [384]. In the previous version of these guidelines, a weak recommendation was issued for using invasive monitoring of arterial blood pressure over non-invasive monitoring [12]. Since then, no new relevant evidence became available. Large, randomised trials that compare arterial blood pressure monitoring versus non-invasive methods are still lacking. In view of the low complication rate and likely higher accuracy of blood pressure measurement, the benefits of arterial catheters probably outweigh the risks. However, the potentially limited resources in some countries and the lack of high-quality studies need to be considered. Therefore, the panel issued a weak recommendation in favour of arterial catheter placement. Arterial catheters should be removed as soon as continuous haemodynamic monitoring is no longer required to minimise the risk of complications.

The prompt initiation of vasopressors to restore blood pressure is an integral component of the management 
of septic shock. Vasopressors have been traditionally administered via a central venous access due to concerns of extravasation, local tissue ischaemia and injury if administered peripherally. However, the process of securing central venous access can be time consuming and requires specialised equipment and training that may not be available in under resourced settings even in high income countries, leading to a delayed initiation of vasopressors [385]. Large randomised trials that compare central and peripheral catheters for initial infusion of vasopressor are lacking. A small study $(n=263)$ randomly allocated patients to receive peripheral vascular access or a central access [386]. The need for vasopressor was the indication for venous access in $70 \%$ of the patients. The incidence of major catheterrelated complications was higher in those randomised to peripheral venous lines with no significant difference in the incidence of minor catheter-related complication. The most common peripheral venous line complication was difficulty in placement. Almost half of the patients assigned to the peripheral access group did not need a central line throughout their ICU stay. Other authors also showed that central lines could be avoided by peripheral line insertion [387]. The administration of vasopressors through peripheral IV catheters is generally safe. A recent systematic review showed that extravasation occurred in $3.4 \%$ (95\% CI $2.5-4.7 \%$ ) of patients with no reported episodes of tissue necrosis or limb ischaemia [388]. Most of the studies reported no need for active treatment of the extravasation, and a systematic review concluded that most patients who experience extravasation events have no long-term sequelae [389]. Extravasation may occur more frequently if vasopressors are infused distally to the antecubital fossa; a meta-analysis showed that $85 \%$ of reported extravasation events occurred when vasopressors were infused by a catheter that was located distal to the antecubital fossa [389]. The occurrence of local tissue injury may be more likely with prolonged administration of vasopressors. Administration of vasopressors for a short period of time $(<6 \mathrm{~h})$ in a well-placed peripheral catheter proximal to the antecubital fossa is unlikely to cause local tissue injury [389].

The time to initiation of vasopressors may be shorter if peripheral access is used. A post-hoc analysis of the ARISE trial showed that $42 \%$ of patients had vasopressors initiated via a peripheral catheter with a shorter time to initiation of vasopressors (2.4 [1.3-3.9] vs. $4.9 \mathrm{~h}$ [3.5-6.6], $p<0.001)$ [385]. Moreover, most patients who had vasopressors started peripherally achieved a MAP $>65 \mathrm{mmHg}$ within $1 \mathrm{~h}$. Delay in vasopressor initiation and achieving MAP of 65 is associated with increased mortality [390, 391].
Given the low complication rate of peripheral vasopressors and the possibility of restoring blood pressure faster, the benefits of initiating vasopressors for a short period of time in a vein proximal to the antecubital fossa probably outweigh the risks. Therefore, we issued a weak recommendation in favour of the rapid initiation of vasopressors peripherally. If the infusion of vasopressors is still needed after a short period of time, as soon as practical and if resources are available, they should be infused through a central venous access to minimise the risk of complications. The lack of availability and expertise in placement of central venous catheters in different settings is an important consideration [55]. Though data are generally sparse on the latter, a study of mostly senior resident doctors in Nigeria concluded that knowledge of central venous catheter placement was limited [392]. Though the panel suggests peripheral administration of norepinephrine as a temporizing measure until a central venous catheter can be placed, its longer-term central administration may not be possible in some settings. Larger prospective studies are needed to provide better evidence on the adequacy and safety of peripheral lines in this scenario.

\section{Fluid balance \\ Recommendation \\ 45. There is insufficient evidence to make a recommendation on the use of restrictive versus liberal fluid strategies in the first $24 \mathrm{~h}$ of resus- citation in patients with sepsis and septic shock who still have signs of hypoperfusion and volume depletion after initial resuscitation \\ Remarks \\ Fluid resuscitation should be given only if patients present with signs of hypoperfusion}

\section{Rationale}

The current literature does not provide clear guidance about the best fluid strategy following the initial resuscitation bolus of fluids. The four largest clinical trials in sepsis resuscitation used moderate to large amounts of fluids in the first $72 \mathrm{~h}$. Although Rivers [393] administered over 13 L of fluids, ProCESS[64], ARISE [65] and ProMISe [66] administered approximately 7-8 $\mathrm{L}$ in the usual care groups with a reported low mortality rate. However, recent evidence suggests that IV fluids used to restore organ perfusion may damage vascular integrity and lead to organ dysfunction [394]. Data from observational studies have shown an association of high-volume fluid resuscitation and increased mortality, but these studies are likely affected by unmeasured variables (i.e. the administration of higher amounts of fluids to sicker patients) [395, 396]. Recent data emerging from Africa showed that higher volume fluid resuscitation in adults was associated with 
increased mortality, but the generalizability of these data is limited due to the high prevalence of HIV/AIDS and malnutrition in the patients enrolled and the resource-scarce conditions with limited access to ICUs [69].

The current evidence evaluating a restrictive IV fluid strategy in the management of septic patients varies with respect to the inclusion criteria, the definition of restrictive and liberal fluid strategies, the criteria guiding the administration of additional IV fluids (e.g., perfusion parameters vs. haemodynamic variables), and the duration of the interventions [397-401]. Moreover, the primary outcomes were mostly related to IV fluid volumes administered during the study period and given the small sample sizes, they were not powered to identify differences in patientcentered outcomes. The ongoing Crystalloid Liberal or Vasopressors Early Resuscitation in Sepsis (CLOVERS) trial and the Conservative vs liberal fluid therapy in septic shock (CLASSIC) trial will shed some light to this matter $[402,403]$. Given the quality of the evidence and the variability among existing studies, the panel issued no recommendation for either restrictive or liberal fluid management in the first $24 \mathrm{~h}$ of resuscitation after the initial fluid bolus in patients with sepsis and septic shock. However, it is important to emphasise this discussion does not affect the recommendation for the initial IV fluid bolus and that the administration of IV fluids after the initial fluid bolus should be guided by perfusion parameters and not only by a response in haemodynamic variables.

\section{Ventilation}

Oxygen targets

\section{Recommendation}

46. There is insufficient evidence to make a recommendation on the use of conservative oxygen targets in adults with sepsis-induced hypoxemic respiratory failure

\section{Rationale}

Patients who are undergoing mechanical ventilation in the ICU often receive a high fraction of inspired oxygen and have a high arterial oxygen tension. The conservative use of oxygen may reduce oxygen exposure and diminish lung and systemic oxidative injury. The evidence for the use of conservative oxygen targets (generally defined as $\mathrm{PaO}_{2}$ 55-70 mmHg; $\mathrm{SpO}_{2} 88-92 \%$ ) and therapy in patients with sepsis is limited, with three randomised trials in the critically ill population [404-406]. In the 1000-participant ICU-ROX trial [405], conservative oxygen therapy did not significantly affect the primary outcome, which was the number of ventilator-free days, compared with liberal oxygen therapy for ventilated adults in ICU. Mortality at 90 and 180 days did not differ. These findings are at variance with the results of a previous single-centre trial, which was stopped early after an unplanned interim analysis. In that trial, conservative oxygen therapy in the ICU was associated with a markedly lower rate of death than usual oxygen therapy [404]. In a recent systematic review and meta-analysis of multiple clinical syndromes, investigators found that a conservative oxygen strategy was associated with a lower rate of death in acutely ill adults than a liberal oxygen strategy [407]. However, in a post hoc analysis of the ICU-ROX trial including adults with sepsis, point estimates for the treatment effect of conservative oxygen therapy on 90-day mortality raise the possibility of clinically important harm [408]. The LOCO-2 study was terminated early by the data safety and monitoring board and reported no difference in 28-day survival in ARDS patients managed with a conservative oxygenation strategy [409]. There are several ongoing trials of conservative oxygen targets that will inform clinical practice in the future. At this point in time, there is insufficient evidence to make an evidencebased recommendation.

\section{High-flow nasal oxygen therapy Recommendation \\ 47. For adults with sepsis-induced hypoxemic respiratory failure, we sug- gest the use of high flow nasal oxygen over non-invasive ventilation Weak recommendation, low quality of evidence}

\section{Rationale}

Acute hypoxemic respiratory failure can result from causes of sepsis such as pneumonia or non-pulmonary infections resulting in ARDS. Patients presenting with hypoxia without hypercapnia are treated with high concentrations of inhaled oxygen which may be delivered conventionally with interfaces including nasal prongs, facemask with reservoir or Venturi mask.

Advanced interventions for patients with severe hypoxia requiring escalation of support include noninvasive ventilation (NIV) or high flow oxygen. Both therapies avoid the complications of intubation and invasive mechanical ventilation and promote patient interaction. In addition to improving gas exchange, NIV may help to reduce work of breathing in select patients. However, NIV use can be associated with development of complications including increased risk of gastric insufflation and aspiration, facial skin breakdown, excessively high tidal volumes as well as patient discomfort related to inability to eat or effectively phonate during therapy.

High flow nasal cannula (HFNC) is a non-invasive, high concentration oxygen delivery interface that confers warming and humidification of secretions, high flow rates to better match patient demand, washout of nasopharyngeal dead space, and modest positive airway pressure 
effect. The single inspiratory limb of HFNC allows for airflows as high as $60 \mathrm{~L}$ per minute to achieve inspired oxygen fractions $\left(\mathrm{FiO}_{2}\right)$ as high as $95-100 \%$. However, HFNC is less effective at reducing work of breathing and supplying a moderate or higher level of PEEP [410]. Complications with HFNC are possible; however, they are usually self-limited and do not require discontinuing therapy.

When comparing the strategies of NIV versus HFNC for acute hypoxemic respiratory failure despite conventional oxygen, a single, large randomised trial has been conducted for direct comparison [411]. Although the primary outcome of intubation rate at 28 days was not different, this study demonstrated improved 90-day survival with HFNC compared with NIV (OR 0.42; 95\% CI 0.21-0.85) and HFNC patients experienced significantly more days free of mechanical ventilation during a 28-day study period [411]. In a post hoc analysis of patients with severe hypoxemia $\left(\mathrm{PaO}_{2} / \mathrm{FiO}_{2} \leq 200 \mathrm{mmHg}\right)$ from the above trial, HFNC resulted in lower intubation rates compared with NIV (35 versus 58 percent, respectively). A systematic review and meta-analysis of nine RCTs [2] showed that HFNC reduces intubation compared with conventional oxygen (RR 0.85 ; 95\% CI 0.74-0.99) but does not affect the risk of death or ICU length of stay [412-414]. However, the NIV technique was not standardised and the experience of the centers varied.

Although the quality of evidence is low, the benefits of a trial of HFNC for the sepsis patient with non-hypercapnic progressive hypoxia over NIV seems justified. Patients requiring HFNC for acute hypoxemic respiratory failure are at high risk of requiring intubation; therefore, such trials must be accompanied by careful surveillance for ventilatory failure.

\section{Non-invasive ventilation}

\section{Recommendation}

48. There is insufficient evidence to make a recommendation on the use of non-invasive ventilation in comparison to invasive ventilation for adults with sepsis-induced hypoxemic respiratory failure

\section{Rationale}

When directly compared to invasive positive pressure ventilation, NIV may be able to achieve similar physiologic benefits including improved gas exchange and reduced work of breathing in select patients, while avoiding complications associated with intubation, invasive ventilation, and accompanying sedation. In contrast, NIV can cause mask-related discomfort, unrecognised patient-ventilator asynchrony due to leaks, and gastric insufflation. The main risk of NIV for the indication of acute respiratory failure is the potential for delaying needed intubation and increasing the risk of an interval aspiration events. Studies have suggested that NIV failure is an independent risk factor for mortality specifically in this population, although careful patient selection may reduce this risk $[415,416]$.

Patients with sepsis-induced hypoxemic respiratory failure may or may not have a competing chronic respiratory disease (ex. COPD, obesity) and the use of NIV for the rescue of patients with exclusively acute hypoxic respiratory failure ("de novo respiratory failure") is less well studied, but not uncommon. For example, the LUNG SAFE trial demonstrated that NIV was used in $15 \%$ of patients with ARDS with varying failure and mortality rates, depending on ARDS severity [417].

A few small RCTs have shown benefit with NIV for early or mild ARDS or de novo hypoxic respiratory failure $[418,419]$. Since the last guideline distribution, only one additional study was added for analysis [420]. Due to small number of patients studied, low quality of evidence, uncertainty regarding whether clinicians can identify hypoxic patients in respiratory failure in whom NIV might be beneficial, and observational data that suggest the potential for harm with NIV in this setting, no clear recommendation can be made. If NIV is used for patients with sepsis-associated hypoxic respiratory failure, we suggest monitoring for an early reduction in work of breathing and close monitoring of tidal volumes [421].

\section{Protective ventilation in acute respiratory distress syndrome (ARDS)}

\section{Recommendation}

49. For adults with sepsis-induced ARDS, we recommend using a low tidal volume ventilation strategy $(6 \mathrm{~mL} / \mathrm{kg})$, over a high tidal volume strategy $(>10 \mathrm{~mL} / \mathrm{kg})$

Strong recommendation, high quality of evidence

\section{Rationale}

This recommendation is the same as that of the previous guidelines. Of note, the studies that guide the recommendations in this section enrolled patients using criteria from the American-European Consensus Criteria Definition for Acute Lung Injury and ARDS [422]. For the current document, we used the 2012 Berlin definition and the terms mild, moderate, and severe ARDS $\left(\mathrm{PaO}_{2} / \mathrm{FiO}_{2} \leq 300, \leq 200\right.$, and $\leq 100 \mathrm{~mm} \mathrm{Hg}$, respectively $)$ [423]. Several multicentre RCTs have been performed in patients with established ARDS to evaluate the effects of limiting inspiratory pressure through moderation of tidal volume [424-427]. These studies showed differing results, which may have been caused by differences in airway pressures in the treatment and control groups $[423,424,428]$. 
Several meta-analyses suggest decreased mortality in patients with a pressure- and volume-limited strategy for established ARDS [353, 354]. The largest trial of a volume- and pressure-limited strategy showed $9 \%$ absolute decrease in mortality in ARDS patients ventilated with tidal volumes of $6 \mathrm{~mL} / \mathrm{kg}$ compared with $12 \mathrm{~mL} / \mathrm{kg}$ predicted body weight (PBW), and aiming for plateau pressure $\leq 30 \mathrm{~cm} \mathrm{H}_{2} \mathrm{O}$ [424].

The use of lung-protective strategies for patients with ARDS is supported by clinical trials and has been widely accepted; however, the precise tidal volume for an individual ARDS patient requires adjustment for factors such as the plateau pressure, the selected positive end-expiratory pressure (PEEP), thoracoabdominal compliance, and the patient's breathing effort. Patients with profound metabolic acidosis, high minute ventilation, or short stature may require additional manipulation of tidal volumes. Some clinicians believe it may be safe to ventilate with tidal volumes $>6 \mathrm{~mL} / \mathrm{kg}$ PBW as long as plateau pressure can be maintained $\leq 30 \mathrm{~cm} \mathrm{H}_{2} \mathrm{O}[429,430]$. The plateau pressure is only truly valuable if the patient is passive during the inspiratory hold. Conversely, patients with very stiff chest/ abdominal walls and high pleural pressures may tolerate plateau pressures $>30 \mathrm{~cm} \mathrm{H}_{2} \mathrm{O}$ because transpulmonary pressures will be lower. A retrospective study suggested that tidal volumes should be lowered even with plateau pressures $\leq 30 \mathrm{~cm} \mathrm{H}_{2} \mathrm{O}$ [431] because lower plateau pressures were associated with reduced hospital mortality [432]. A recent patient-level mediation analysis suggested that a tidal volume that results in a driving pressure (plateau pressure minus set PEEP) below $12-15 \mathrm{~cm} \mathrm{H}_{2} \mathrm{O}$ may be advantageous in patients without spontaneous breathing efforts [433]. Prospective validation of tidal volume titration by driving pressure is needed before this approach can be recommended. Tidal volumes $>6 \mathrm{cc} / \mathrm{kg}$ coupled with plateau pressures $>30 \mathrm{~cm}$ $\mathrm{H}_{2} \mathrm{O}$ should be avoided in ARDS. Clinicians should use as a starting point the objective of reducing tidal volume over 1-2 $\mathrm{h}$ from its initial value toward the goal of a "low" tidal volume $(\approx 6 \mathrm{~mL} / \mathrm{kg} \mathrm{PBW})$ achieved in conjunction with an end-inspiratory plateau pressure $\leq 30 \mathrm{~cm} \mathrm{H}_{2} \mathrm{O}$. If plateau pressure remains $>30 \mathrm{~cm} \mathrm{H}_{2} \mathrm{O}$ after reduction of tidal volume to $6 \mathrm{~mL} / \mathrm{kg}$ PBW, tidal volume may be further reduced to as low as $4 \mathrm{~mL} / \mathrm{kg}$ PBW. The clinician should keep in mind that very low tidal volumes may result in significant patient-ventilatory dyssynchrony and patient discomfort. Respiratory rate should be increased to a maximum of 35 breaths/min during tidal volume reduction to maintain minute ventilation. Volume- and pressure-limited ventilation may lead to hypercapnia even with these maximum-tolerated set respiratory rates; this appears to be tolerated and safe in the absence of contraindications (e.g., high intracranial pressure, sickle cell crisis). No single mode of ventilation (pressure control, volume control) has consistently been shown to be advantageous when compared with any other that respects the same principles of lung protection.

Recommendation
50. For adults with sepsis-induced severe ARDS, we recommend using
an upper limit goal for plateau pressures of $30 \mathrm{~cm} \mathrm{H}_{2} \mathrm{O}$, over higher
plateau pressures
Strong recommendation, moderate quality of evidence

\section{Rationale}

This recommendation is unchanged from the previous guidelines, as no new trials evaluating plateau pressure have been published since then. Of note, the 3 RCTs that guide this recommendation [424, 426, 427] enrolled patients using the criteria from the American-European Consensus Criteria Definition for Acute Lung Injury and ARDS [422] whereas the current document use the 2012 Berlin definition and the terms mild, moderate, and severe ARDS $\left(\mathrm{PaO}_{2} / \mathrm{FiO}_{2} \leq 300, \leq 200\right.$, and $\leq 100 \mathrm{~mm} \mathrm{Hg}$, respectively) [423]. These three RCTS compared a strategy of low tidal volume and limited plateau pressure with a strategy using higher tidal volume and plateau pressure; pooled data suggest reduced mortality (RR $0.83 ; 95 \% \mathrm{CI}$ $0.70-0.97$ ) and more ventilator-free days (MD 1.8 days; 95\% CI 0.35-3.25) in patients managed with low plateau pressures.

A recent systematic review which included five RCTs also identified a strong relationship between plateau pressure and mortality [434]. The recommendation is also supported by observational data. LUNGSAFE, a large international observational study, which reported that plateau pressure correlated with mortality; however, the relationship between the two was not evident when plateau pressure was below $20 \mathrm{~cm} \mathrm{H}_{2} \mathrm{O}$ [435]. A secondary analysis of five observational studies identified a plateau pressure cut-off value of $29 \mathrm{~cm} \mathrm{H}_{2} \mathrm{O}$, above which an ordinal increment was accompanied by an increment of risk of death [436]. We therefore recommend that the upper limit goal for plateau pressure should be less than $30 \mathrm{~cm} \mathrm{H}_{2} \mathrm{O}$.

\footnotetext{
Recommendation

51. For adults with moderate to severe sepsis-induced ARDS, we suggest using higher PEEP over lower PEEP

Weak recommendation, moderate quality of evidence
} 


\section{Rationale}

The recommendation is unchanged from 2016. Two RCTs [437, 438] were published since the 2016 Guidelines [12, $13]$, but we did not include these trials in the meta-analyses because both studies applied recruitment maneuvers to titrate PEEP levels. Our conclusions did not change in a sensitivity analysis which includes these two trials.

Applying higher PEEP in patients with ARDS may open lung units to participate in gas exchange and may increase $\mathrm{PaO}_{2}$. We included three multicentre RCTs [439-441] and one pilot RCT [442], investigating use of higher PEEP versus lower PEEP strategies in conjunction with low tidal volumes for the management of patients with ARDS. Among patients with ARDS receiving lower VTs, we did not identify a significant benefit for use of a higher PEEP versus lower PEEP strategy for improving mortality $(R R=0.93 ; 95 \%$ CI $0.83-1.03)$, days on mechanical ventilation $(R R=0.00 ; 95 \% C I-1.02$ to 1.02$)$, or ventilator-free days $(\mathrm{RR}=1.48 ; 95 \% \mathrm{CI} 0.19-2.76)$; and there was no increase in the risk of barotrauma $(\mathrm{RR}=1.49$; 95\% CI 0.99-2.23).

A patient-level meta-analysis showed no benefit of higher PEEP in all patients with ARDS; however, patients with moderate or severe ARDS $\left(\mathrm{PaO}_{2} / \mathrm{FiO}_{2} \leq 200 \mathrm{mmHg}\right)$ had decreased mortality with the use of higher PEEP, whereas those with mild ARDS did not [443]. A patientlevel analysis of two of the randomised PEEP trials [440, 441] suggested that patients with ARDS who respond to increased PEEP with improved oxygenation have a lower risk of death; this association was stronger in patients with more severe ARDS $\left(\mathrm{PaO}_{2} / \mathrm{FiO}_{2}<150 \mathrm{mmHg}\right)$ compared with patients with less severe ARDS [444].

The optimal method of selecting a higher PEEP level is not clear. One option is to titrate PEEP according to bedside measurements of thoracopulmonary compliance with the objective of obtaining the best compliance or lowest driving pressure, reflecting a favourable balance of lung recruitment and overdistension [445]. The second option is to titrate PEEP upward while the patient is receiving a tidal volume of $6 \mathrm{~mL} / \mathrm{kg} \mathrm{PBW}$, until the plateau airway pressure is $28 \mathrm{~cm} \mathrm{H}_{2} \mathrm{O}$ [441]. A third option is to use a $\mathrm{PEEP} / \mathrm{FiO}_{2}$ titration table that titrates PEEP based on the combination of $\mathrm{FiO}_{2}$ and PEEP required to maintain adequate oxygenation [439-441]. A PEEP $>5 \mathrm{~cm} \mathrm{H}_{2} \mathrm{O}$ is usually required to avoid lung collapse [446]. Esophageal pressure guided PEEP titration has been evaluated in two trials [447, 448]. While the pilot study suggested benefit [448], the subsequent 200 patient multicentre RCT that compared PEEP titration guided by esophageal $\left(P_{\mathrm{ES}}\right)$ measurement versus empirical high $\mathrm{PEEP}-\mathrm{FiO}_{2}$ titration, showed no significant difference in a composite outcome of death and days free from mechanical ventilation through day 28 [449].
Low tidal volume in non-ARDS respiratory failure

\section{Recommendation}

52. For adults with sepsis-induced respiratory failure (without ARDS), we suggest using low tidal volume as compared to high tidal volume ventilation

Weak recommendation, low quality of evidence

\section{Rationale}

Previous versions of SSC guidelines issued a strong recommendation with a moderate-quality evidence for using low tidal volume (Vt) ventilation (Vt $4-8 \mathrm{~mL} /$ $\mathrm{kg}$ of predicted body weight), over higher tidal volumes $(\mathrm{Vt}>8 \mathrm{~mL} / \mathrm{kg})$ in the management of patients with ARDS $[12,13,226]$. There is not as strong an evidence base, however, for the patients presenting with acute respiratory failure requiring mechanical ventilation who do not fulfil the criteria for ARDS. A 2015 systematic review and meta-analysis found a reduction in the risk of a composite endpoint of ARDS or pneumonia during the hospital stay in the low tidal volume ventilation group compared to the high tidal volume ventilation group (RR $0.72 ; 95 \%$ CI 0.52-0.98) [450]. Our analysis of three RCTs (1129 patients) showed no difference in mortality with low $V_{\mathrm{t}}$ ventilation (RR 1.07; 95\% CI 0.91-1.26), with a trend towards lower risk of developing ARDs (RR 0.59; 95\% CI 0.34-1.02) (Supplementary Appendix 4).

There are limited data on ventilation strategies for patients with sepsis-induced respiratory failure who do not meet criteria for ARDS. However, sepsis is an independent risk factor for the development of ARDS, and delays in diagnosing ARDS may result in delayed use of low tidal volumes. We therefore suggest that low tidal volume ventilation be used in all patients with sepsis who are receiving mechanical ventilation in order to avoid underuse or delayed use of this intervention. Furthermore, the use of low tidal volume ventilation avoids the risk of promoting ventilator induced lung injury in septic patients in whom the diagnosis of ARDS has been missed.

\section{Recruitment manoeuvres}

\section{Recommendations}

53. For adults with sepsis-induced moderate-severe ARDS, we suggest using traditional recruitment maneuvers

Weak recommendation, moderate quality of evidence

54. When using recruitment maneuvers, we recommend against using incremental PEEP titration/strategy

Strong recommendation, moderate quality of evidence

\section{Rationale}

Many strategies exist for treating refractory hypoxemia in patients with severe ARDS [451]. Temporarily raising 
transpulmonary pressure may facilitate opening atelectatic alveoli to permit gas exchange [446], but could also over distend aerated lung units leading to ventilatorinduced lung injury and transient hypotension. Since the publication of the previous SSC Guidelines, two important RCTs were published both of which utilised a "non-traditional" approach to recruitment maneuvers. Instead of the "traditional" recruitment maneuver which consists of the application of sustained continuous positive airway pressure (e.g., $30-40 \mathrm{~cm} \mathrm{H}_{2} \mathrm{O}$ for $30-40 \mathrm{~s}$ ), both trials conducted lung recruitment with incremental PEEP levels, followed by a decremental PEEP titration according to either best respiratory-system static compliance [452] or oxygen saturation [437]. When the incremental PEEP recruitment studies are analysed separately from studies utilizing traditional recruitment maneuvers, recruitment with incremental PEEP is associated with increased 28-day mortality RR 1.12; 95\% CI 1.00-1.25), which justifies the strong recommendation against using incremental PEEP titration for recruitment. Traditional recruitment maneuvers appear to improve 28-day mortality (RR 0.79; 95\% CI 0.64-0.96) in patients with ARDS (Supplementary Appendix 4). Although the effects of recruitment maneuvers improve oxygenation initially, the effects can be transient [453]. Selected patients with severe hypoxemia may benefit from recruitment maneuvers in conjunction with higher levels of PEEP, but little evidence supports the routine use in all ARDS patients, so we have focused our recommendations to patients with moderate-to-severe ARDS [453]. Any patient receiving recruitment maneuvers should be monitored closely and recruitment maneuvers should be discontinued if deterioration in clinical status is observed.

\section{Prone ventilation}

\section{Recommendation}

55. For adults with sepsis-induced moderate-severe ARDS, we recommend using prone ventilation for more than $12 \mathrm{~h}$ daily

Strong recommendation, moderate quality of evidence

\section{Rationale}

There were no new randomised, controlled trials evaluating the use of prone ventilation in sepsis induced severe ARDS published since the 2016 guidelines. Therefore, no change in the recommendation was made. In 2017, a meta-analysis was published [454] that was updated from a previous meta-analysis published in 2010 [455], to which only 1 study, the PROSEVA trial, published in 2013 [456] was added. This repeat meta-analysis confirmed the results from the previous published work: In patients with ARDS and a $\mathrm{PaO}_{2} / \mathrm{FiO}_{2}$ ratio $<200$, the use of prone compared with supine position within the first $36 \mathrm{~h}$ of intubation, when performed for $>12 \mathrm{~h}$ a day, showed improved survival. Meta-analysis including this study demonstrated reduced mortality in severe ARDS patients treated with prone compared with supine position (RR 0.74; 95\% CI 0.56-0.99) as well as improved oxygenation as measured by change in $\mathrm{PaO}_{2} / \mathrm{FiO}_{2}$ ratio (median 23.5 higher; 95\% CI 12.4-34.5 higher) [454]. Most patients respond to the prone position with improved oxygenation and may also have improved lung compliance [457-459]. While prone position may be associated with potentially life-threatening complications including accidental removal of the endotracheal tube, this was not evident in pooled analysis (RR 1.09; 95\% CI 0.85-1.39). However, prone position was associated with an increase in pressure sores (RR 1.22; 95\% CI 1.05-1.41) [460, 461], and some patients have contraindications to the prone position $[460,461]$.

\section{Neuromuscular blocking agents Recommendation \\ 56. For adults with sepsis induced moderate-severe ARDS, we suggest using intermittent NMBA boluses, over NMBA continuous infusion Weak recommendation, moderate quality of evidence}

\section{Rationale}

The most common indication for neuromuscular blocking agents (NMBAs) use in the ICU is to facilitate mechanical ventilation [462]. These drugs may improve chest wall compliance, prevent respiratory dyssynchrony, and reduce peak airway pressures [463]. In addition, use of NMBA may reduce oxygen consumption by decreasing the work of breathing [464]. In the SSC 2016 guidelines, we issued a weak recommendation for using NMBA infusion for $48 \mathrm{~h}$ in sepsis-induced moderate to severe ARDS $[12,13]$. This recommendation was based on a metaanalysis of 3 trials that examined the role of NMBAs in ARDS [465-467], showing reduced risks of death (RR 0.72; 95\% CI 0.58-0.91) and barotrauma (RR 0.43; 95\% CI $0.20-0.90$ ) with the use of cisatracurium infusion [468].

Since then, several RCTs have been published [469471], the largest of which is the ROSE Trial [471]. Due to the presence of significant statistical and clinical heterogeneity, a meta-analysis of all seven trials was not appropriate. A continuous NMBA infusion did not improve mortality when compared to a light sedation strategy with as needed NMBA boluses but no continuous infusion (RR 0.99; 95\% CI 0.86-1.15). On the other 
hand, continuous NMBA infusion reduced mortality when compared to deep sedation with as needed NMBA boluses (RR 0.71; 95\% CI 0.57-0.89). Overall, continuous NMBA infusion reduced the risk of barotrauma (RR 0.55; 95\% CI 0.35-0.85), but the effect on ventilator-free days, duration of mechanical ventilation, and ICU-acquired weakness was unclear $[472,473]$.

Given the uncertainty that still exists pertaining to these important outcomes and the balance between benefits and potential harms, the panel issued a weak recommendation favouring intermittent NMBA boluses over a continuous infusion. Importantly, if NMBAs are used, clinicians must ensure adequate patient sedation and analgesia [191, 474]. Recently updated clinical practice guidelines are also available for specific guidance [472].

\section{Extracorporeal membrane oxygenation (ECMO)}

Recommendation
57. For adults with sepsis-induced severe ARDS, we suggest using veno-
venous (W) ECMO when conventional mechanical ventilation fails in
experienced centers with the infrastructure in place to support its use
Weak recommendation, low quality of evidence

\section{Rationale}

Venovenous (VV) extracorporeal membrane oxygenation $(E C M O)$ is used in patients with severe acute respiratory failure to facilitate gas exchange in the setting of refractory hypoxaemia or hypercapnic respiratory acidosis [475]. It may also be used to facilitate a reduction in the intensity of mechanical ventilation. The evidence for the use of VVECMO in sepsis-induced ARDS is limited, with two RCTs completed in the last 10 years to assess the potential efficacy of VV ECMO for severe ARDS [476, 477]. The inclusion criteria of the trials were strict and focused on a very sick population of patients with severe ARDS refractory to conventional ventilation strategies and other rescue therapies such as prone position. The evidence in this guideline was downgraded to very low quality due to indirectness.

There were methodological limitations of the included studies. In one trial, all intervention participants were treated at one centre, which may have inflated the effect size because the centre specialised in ECMO management [477]. In addition, some of the participants in this trial did not receive the intervention [477]. However, one recent systematic review found that VV ECMO delivered at expert centers reduced mortality for patients with severe ARDS [475]. In clinical practice, patient selection is important and usually discussed prior to initiation of ECMO at an ECMO centre. Cost and equity are substantial issues; and registry data will be very important to document longer-term outcomes in these patients outside of the clinical trial context.

\section{Additional therapies \\ Corticosteroids \\ Recommendation \\ 58. For adults with septic shock and an ongoing requirement for vaso- pressor therapy we suggest using IV corticosteroids \\ Weak recommendation; moderate quality of evidence \\ Remark \\ The typical corticosteroid used in adults with septic shock is IV hydrocor- tisone at a dose of $200 \mathrm{mg} /$ day given as $50 \mathrm{mg}$ intravenously every $6 \mathrm{~h}$ or as a continuous infusion. It is suggested that this is commenced at a dose of norepinephrine or epinephrine $\geq 0.25 \mathrm{mcg} / \mathrm{kg} / \mathrm{min}$ at least $4 \mathrm{~h}$ after initiation}

\section{Rationale}

In the 2016 guidance, the accumulated evidence did not support a recommendation for their use if adequate fluid resuscitation and vasopressor therapy were able to restore haemodynamic stability [12, 13] Since then, three large RCTs have been published [354, 478, 479]. An updated meta-analysis [480] found systemic corticosteroid to accelerate resolution of shock (MD 1.52 days; 95\% CI 1.71-1.32). A meta-analysis conducted for this guideline revision (Supplementary Appendix 5) found an increase vasopressor-free days (MD 1.5 days; $95 \%$ CI $0.8-$ 3.11 days); however, corticosteroid use increased neuromuscular weakness (RR 1.21; 95\% CI 1.01-1.45), without a clear effect on short- or long-term mortality.

The overall quality of evidence was moderate. The panel judged the desirable effects (shock resolution, vasopressor free days) to outweigh the undesirable effects of low dose corticosteroid. This observation, when taken into consideration with the resources required, cost of the intervention, and feasibility supported a weak recommendation in favour of using low dose corticosteroid therapy in septic shock.

The optimal dose, timing of initiation, and duration of corticosteroids remain uncertain; recent RCTs used $200 \mathrm{mg}$ per day of IV hydrocortisone in divided doses $[354,479]$. The three trials [354, 478, 479] also used different inclusion criteria: in ADRENAL [479] eligible patients were those on any dose of vasopressor or inotrope for $\geq 4 \mathrm{~h}$ to maintain a MAP $>60 \mathrm{mmHg}$, and present at the time of randomisation. In APROCCHSS [478] the dose of vasopressor was $\geq 0.25 \mu \mathrm{g} / \mathrm{kg} / \mathrm{min}$ or $\geq 1 \mathrm{mg} / \mathrm{h}$ of norepinephrine or epinephrine, or any other vasopressor for at least $6 \mathrm{~h}$ to maintain a MAP $\geq 65 \mathrm{mmHg}$. In the ADRENAL [479] study, hydrocortisone was administered for a maximum of seven days or until ICU discharge or death; in APROCCHSS [478] hydrocortisone was administered for seven days; in VANISH [354] $200 \mathrm{mg}$ of hydrocortisone was administered daily for 5 days and then tapered over further 6 days. 
Our recommendation focuses on adults with septic shock and ongoing requirement for vasopressor therapy. We defined ongoing requirement as a dose of norepinephrine or epinephrine $\geq 0.25 \mathrm{mcg} / \mathrm{kg} / \mathrm{min}$ for at least $4 \mathrm{~h}$ after initiation to maintain the target MAP. The dose of hydrocortisone is typically $200 \mathrm{mg} /$ day. No dose response benefit was seen in a prior systematic review and meta-analysis [480].

\section{Blood Purification}

\section{Recommendations}

59. For adults with sepsis or septic shock, we suggest against using polymyxin B haemoperfusion

Weak recommendation; Iow quality of evidence

60. There is insufficient evidence to make a recommendation on the use of other blood purification techniques

\section{Rationale}

Haemoperfusion refers to the circulation of blood through an extracorporeal circuit that contains an adsorbent containing cartridge. The previous guidelines made no recommendation regarding the use of blood purification techniques $[12,13]$. The updated literature search for guideline identified one new relevant RCT [481].

The most widely investigated technique involves the use of polymyxin B-immobilised polystyrene-derived fibers. Randomised trials of this technique have been previously summarised in a systematic review and metaanalysis [482]. An updated meta-analysis of all available RCTs (Supplementary Appendix 5) demonstrated a possible reduction in mortality (RR $0.87 ; 95 \%$ CI $0.77-0.98$, low quality), however this finding was challenged by sensitivity analyses: after excluding high risk of bias trials the risk ratio is 1.14 (95\% CI 0.96-1.36); and after excluding trials published prior to 2010 we observed higher mortality with haemoperfusion (RR 1.23; 95\% CI 1.04-1.46). Overall, the quality of evidence is judged as low (Supplementary Appendix 5).

Substantial uncertainty as to any beneficial effect exists and the frequency of undesirable effects is reported in few trials. Polymyxin B haemoperfusion is expensive, resource intensive, potentially reduces health equity, and is infeasible in low-income economies. All considered, the panel issued a weak recommendation against the use of polymyxin B haemoperfusion therapy.

We did not identify new evidence on other modalities such as haemofiltration, combined haemoperfusion and haemofiltration or plasma exchange. Accordingly, no recommendation regarding the use of these modalities is made. This is unchanged from the 2016 guidelines. Since the analysis new data has emerged, but at this stage was not sufficient for us to re-consider the recommendation [483].
Further research is needed to determine the effect of various blood purification techniques on patient outcomes.

Red blood cell (RBC) transfusion targets
Recommendation
61. For adults with sepsis or septic shock, we recommend using a restric-
tive (over liberal) transfusion strategy
Strong recommendation; moderate quality of evidence
Remark
A restrictive transfusion strategy typically includes a haemoglobin
concentration transfusion trigger of $70 \mathrm{~g} / \mathrm{L}$; however, RBC transfu-
sion should not be guided by haemoglobin concentration alone.
Assessment of a patient's overall clinical status and consideration of
extenuating circumstances such as acute myocardial ischaemia, severe
hypoxemia or acute haemorrhage is required

\section{Rationale}

The previous guidance was informed by two RCTs [484, 485]. The Transfusion Requirements in Septic Shock (TRISS) trial addressed a transfusion threshold of $70 \mathrm{~g} / \mathrm{L}$ versus $90 \mathrm{~g} / \mathrm{L}$ in 1000 septic shock patients after admission to the ICU. The results showed similar 90-day mortality, ischaemic events, and use of life support in the two treatment groups with fewer transfusions in the lowerthreshold group. The Transfusion requirements in in Critical Care trial (TRICC), which compared a restrictive transfusion threshold of $70 \mathrm{~g} / \mathrm{L}$ versus $100 \mathrm{~g} / \mathrm{L}$ in 838 euvolemic ICU patients, demonstrated no difference in the primary outcome (30-day mortality). In the subgroup of 218 patients with sepsis or septic shock 30-day mortality was similar in the two groups $(22.8 \%$ in the restrictive group vs. $29.7 \%$ in the liberal group, $p=0.36$ ).

Our literature search identified a recent systematic review and meta-analysis of RCTs [486] and one new RCT: The Transfusion Requirements in Critically Ill Oncologic Patients (TRICOP) trial [487]. This trial randomised 300 adult cancer patients with septic shock to either a liberal (haemoglobin threshold, $<90 \mathrm{~g} / \mathrm{L}$ ) or restrictive strategy (haemoglobin threshold, $<70 \mathrm{~g} / \mathrm{L}$ ) of $\mathrm{RBC}$ transfusion. At 28 days after randomisation, the mortality rate in the liberal group was $45 \% 67$ patients versus $56 \% 84$ patients in the restrictive group (HR 0.74; 95\% CI $0.53-1.04 ; p=0.08$ ) with no differences in ICU and hospital length of stay. At 90 days after randomisation, mortality rate in the liberal group was lower (59\% vs $70 \%$ ) than in the restrictive group (hazard ratio, $0.72 ; 95 \%$ CI 0.53-0.97).

Our update of the meta-analysis showed no difference in 28-day mortality (OR 0.99 95\% CI 0.67-1.46, moderate quality). This is due to the inclusion of the TRICOP study where lower 28 mortality was observed with a liberal 
strategy. Overall, the quality of evidence was judged moderate.

The overall balance of effects is uncertain and does not favour either the intervention or comparator. However, a restrictive strategy was determined likely beneficial with regards to resources required, cost effectiveness, and health equity considerations. A restrictive strategy is feasible in low- and middle-income countries. The 2016 strong recommendation favouring a restrictive strategy is unchanged; however, the overall quality of evidence changed from strong to moderate.

\section{Immunoglobulins}

Recommendation

62. For adults with sepsis or septic shock, we suggest against using intravenous immunoglobulins

Weak recommendation, low quality of evidence

\section{Rationale}

Patients with sepsis and septic shock may have evidence of hyper-inflammation and immunosuppression [488]. There are no high-quality studies examining the effect of intravenous (IV) immunoglobulins on the outcomes of patients with sepsis or septic shock. The previous guidance was a weak recommendation against their use [12, 13].

Our literature search identified two new RCTs [489, 490] and three meta-analyses [350, 491, 492] evaluating the effects of polyclonal IV immunoglobulins (IVIG) and immunoglobulin M-enriched polyclonal Ig (IVIGM) in patients with sepsis. The updated meta-analyses demonstrated reduced mortality with IVIG (RR 0.73; 95\% CI 0.51-0.91) and IVIGM (RR 0.69; 95\% CI 0.55-0.85), however the quality of evidence is low with many of the included studies at high risks of bias including single-centre trials with small sample size, undefined randomisation, allocation and blinding procedures, different dosing regimens and durations of treatment, different controls and few studies reported adverse events. Furthermore, after excluding high risk of bias studies, the significant reduction in mortality is no longer apparent.

Overall, the balance of effects (beneficial and undesirable) remains uncertain. Intravenous immunoglobulin is also relatively expensive, possibly not cost-effective and may reduce health equity. Its cost also limits its feasibility in countries with low- and middle-income economies. Based on these judgements, clinicians may consider avoiding the routine use of IV immunoglobulins in patients with sepsis and septic shock. Large, multicentre, well-designed, RCTs are needed to resolve the uncertainty regarding the role of immunoglobulin therapies in this patient population.

\section{Stress ulcer prophylaxis \\ Recommendation \\ 63. For adults with sepsis or septic shock, and who have risk factors for gastrointestinal (Gl) bleeding, we suggest using stress ulcer prophy- laxis \\ Weak recommendation, moderate quality of evidence}

\section{Rationale}

Stress ulcers develop in the gastrointestinal (GI) tract of critically ill patients and can be associated with significant morbidity and mortality [493]. In 2016, this guideline recommended stress ulcer prophylaxis for patients with risk factors $[12,13]$.

Our literature search identified one new RCT [494] and the meta-analysis from the previous guideline was updated. This demonstrated no effect on mortality (RR 1.01 95\% CI 0.93-1.10) and a reduction in GI haemorrhage (RR 0.52 95\% CI 0.45-0.61). A sensitivity analysis including only trials at low risk of bias provided similar results. No increase in Clostridoides difficile colitis or pneumonia was observed. However, it was noted that the most recent (and largest) RCT did not demonstrate any effect of pantoprazole versus placebo on 90-day mortality and a composite outcome of clinically important events [494]. A recent meta-analysis published since the finalisation of the literature searches has suggested that there is a higher risk of recurrent Clostridioides difficile infections with proton pump inhibitors [495].

Overall, it was judged that the evidence probably favoured the administration of stress ulcer prophylaxis. This is driven by a modest reduction in gastrointestinal haemorrhage for which there is moderate quality of evidence (Supplementary Appendix 5). While no adverse effects were observed, the quality of evidence for these outcomes was low. Stress ulcer prophylaxis is relatively inexpensive, requires limited resources and is applicable to countries with low-income economies. These judgements support a weak recommendation for the use of stress ulcer prophylaxis in at-risk patients. This represents a downgrading of the strong recommendation based on low-quality evidence made in 2016.

A recent systematic review evaluated risk factors for clinically important GI bleeding [496]. After excluding high risk of bias studies, risk factors included: coagulopathy (relative effect (RE) 4.76; 95\% CI 2.62-8.63), shock (RE 2.60; 95\% CI 1.25-5.42), and chronic liver disease (RE 7.64; 95\% CI 3.32-17.58). The effect of mechanical 
ventilation on clinically important bleeding was unclear (RE 1.93, 0.57-6.50, very low certainty).

Venous thromboembolism (VTE) prophylaxis
Recommendations
64. For adults with sepsis or septic shock, we recommend using phar-
macologic VTE prophylaxis unless a contraindication to such therapy
exists
Strong recommendation, moderate quality of evidence
65. For adults with sepsis or septic shock, we recommend using low
molecular weight heparin (LMWH) over unfractionated heparin (UFH)
for VTE prophylaxis
Strong recommendation, moderate quality of evidence
66. For adults with sepsis or septic shock, we suggest against using
mechanical VTE prophylaxis in addition to pharmacological prophy-
laxis, over pharmacologic prophylaxis alone
Weak recommendation, low quality of evidence

\section{Rationale}

Critically ill patients are at risk for deep vein thrombosis (DVT) as well as pulmonary embolism (PE). The incidence of DVT acquired in the ICU may be as high as $10 \%$ [497], the incidence of acquired PE may be $2-4 \%$ [498, 499].

No new RCT evidence was identified. Our previous meta-analysis demonstrated a significant reduction in both DVT and PE and no increase in bleeding complications.

On balance, the effect favours the intervention with a moderate quality of evidence. The cost of intervention is not large, and it is likely feasible in countries with low- and middle-income economies. These judgements support a recommendation for the use of pharmacologic venous thromboembolism (VTE) prophylaxis unless a contraindication exists. The recommendation is unchanged from the 2016 guidelines.

Our literature review found no new RCT evidence comparing the administration of low molecular weight heparin (LMWH) to unfractionated heparin (UFH). The prior meta-analysis demonstrated significantly lower rates of DVT following the administration of LMWH compared to UFH (RR $0.8495 \%$ CI 0.71-0.98). No difference in the rates of clinically significant bleeding, mortality or PE were observed. The overall quality of evidence was rated as moderate: it was downgraded for imprecision. It was determined that the balance of overall effects favoured LMWH over UFH. Any difference in resources required between the two interventions was considered to be negligible, and LMWH administration was feasible and applicable in countries with low- and middle-income economies. Further, LMWH may have greater consumer acceptance as it requires only one subcutaneous injection daily. These judgements support a recommendation for the use of LMWH over UFH for VTE prophylaxis in patients with sepsis or septic shock. This recommendation is unchanged from the 2016 guidelines.

Combined pharmacologic prophylaxis and mechanical prophylaxis with intermittent pneumatic compression (IPC) and/or graduated stockings may offer another option for patients with sepsis and septic shock. In the 2016 guidelines, a suggestion to use combination therapy whenever possible, was based on indirect and imprecise data $[12,13]$. Our literature search identified one new RCT that compared the combination of mechanical and pharmacological prophylaxis to pharmacological prophylaxis alone [500].

The PREVENT study randomised 2003 critically ill patients to intermittent pneumatic calf compression alone or in combination with pharmacological prophylaxis [500]. No difference in mortality (RR 0.98 95\% CI 0.84-1.13), or the rates of DVT and PE were observed. No difference in lower extremity ischaemia was demonstrated. The study was downgraded during the quality assessment for imprecision. For the outcome of mortality, the quality was assessed as moderate; for other outcomes it was further downgraded for risk of bias.

It was judged that any effects of the intervention (mechanical prophylaxis in addition to pharmacologic), either beneficial or undesirable, were likely trivial (Supplementary Appendix 5). However, there are resource implications and costs associated with the use of mechanical VTE prophylaxis. These, together with the lack of any effect on a patient centered outcome support a weak recommendation against the use of the combination of mechanical and pharmacologic prophylaxis.

It is acknowledged that in some patents with sepsis and septic shock pharmacologic prophylaxis may be contraindicated. These patients may benefit from mechanical VTE prophylaxis. No data for this population exist. Further research is indicated.

\section{Renal replacement therapy}

\section{Recommendations}

67. In adults with sepsis or septic shock and AKI who require renal replacement therapy, we suggest using either continuous or intermittent renal replacement therapy

Weak recommendation, low quality of evidence

68. In adults with sepsis or septic shock and AKI, with no definitive indications for renal replacement therapy, we suggest against using renal replacement therapy

Weak recommendation, moderate quality of evidence

\section{Rationale}

Two systematic reviews and meta-analyses [501, 502] summarised the total body of evidence: they do not show a difference in mortality between patients who receive continuous (CRRT) versus intermittent haemodialysis 
(IHD). The results remained the same when the analysis is restricted to RCTs [502].

Our updated literature search identified no new RCTs but two meta-analysis comparing continuous and intermittent renal replacement therapies [503, 504]. The quality of evidence was judged as low. The balance of effects favoured neither (IHD) nor CRRT. It was acknowledged that the resources required for the interventions vary. In low- and middle-income economies, the specialised equipment, expertise and personal required for continuous modalities may not be available. The recommendation, for either intervention, is unchanged from the 2016 guidelines.

Timing of renal replacement therapy initiation is of importance. Prior research has suggested benefit [505] or harm [506] for "early" versus "delayed" initiation of RRT.

Our search identified a new RCT comparing early versus delayed RRT [507].This trial included 488 patients with AKI and septic shock. It was stopped early, after the second planned interim analysis, for futility. Eligible patients were those with septic shock (within $48 \mathrm{~h}$ of the onset of vasopressor therapy and AKI defined as oliguria $(<0.3 \mathrm{ml} / \mathrm{kg} / \mathrm{h}$ for $\geq 24 \mathrm{~h})$, anuria for $12 \mathrm{~h}$ or more, or a serum creatinine level 3 times baseline accompanied by a rapid increase of $\geq 0.5 \mathrm{mg} / \mathrm{dl}$. Subsequent to the censor date for our literature search, the results of the STARRTAKI trial were published. The trial, which randomised 3000 participants, demonstrated no difference in mortality in those allocated to an accelerated strategy of RRT compared to those allocated to a "standard" strategy. No differential effect was observed in the a priori sepsis subgroup of 1689 patients [508].

The results of this trial were included in an updated meta-analysis (Supplementary Appendix 5). No effect of the timing of initiation of renal replacement therapy on mortality and renal recovery was observed. The IDEALICU trial [507] did not report central venous access device (CVAD) infections: the results for this outcome are unchanged from 2016. The certainty of evidence for the key outcomes of mortality, renal recovery and CVAD infection was a least moderate and was only downgraded for imprecision (Supplementary Appendix 5). Overall, the balance of effects favoured delayed rather than early initiation of RRT. This is principally driven by the higher rate of CVAD infection in the "early" initiation. Therefore, after considering of the resources required, cost and health equity issues, the panel issued a weak recommendation against the use of RRT in patients with sepsis and AKI for increases in creatinine or oliguria alone, and without other absolute indications for dialysis (uremic complications, refractory academia, refractory fluid overload or hyperkalemia).

\section{Glucose control}

\section{Recommendation}

69. For adults with sepsis or septic shock, we recommend initiating insulin therapy at a glucose level of $\geq 180 \mathrm{mg} / \mathrm{dL}(10 \mathrm{mmol} / \mathrm{L})$

Strong recommendation; moderate quality of evidence

Remark

Following initiation of an insulin therapy, a typical target blood glucose range is $144-180 \mathrm{mg} / \mathrm{dL}(8-10 \mathrm{mmol} / \mathrm{L})$

\section{Rationale}

Hyperglycemia (>180 mg/dL), hypoglycemia and increased glycemic variability are associated with increased mortality in critically ill patients [509-511]. The American Diabetes Association, in its most recent recommendations for glycemic control of critically ill patients, recommended the initiation of insulin therapy for persistent hyperglycemia $>180 \mathrm{mg} / \mathrm{dL}$ and thereafter a target glucose range of 140-180 mg/dL [512].

In a single centre study, targeting blood glucose to 80-110 mg/dL reduced ICU mortality [513], however this finding was not reproduced in subsequent multi-centre RCTs $[514,515]$. Meta-analyses also report a higher incidence of hypoglycemia (glucose $<40 \mathrm{mg} / \mathrm{dL}$ ) in critically patients where blood glucose was targeted to $80-110 \mathrm{mg} /$ $\mathrm{dL}[516,517]$. The previous recommendation to commence insulin when two consecutive blood glucose levels are $>180 \mathrm{mg} / \mathrm{dL}$ derives from the NICE-SUGAR trial [518]. A summary of the evidence for this trigger of $>180 \mathrm{mg} / \mathrm{dL}$ is found in the Supplementary Appendix 5. In this version of the guideline, we asked a new question: in adults with sepsis of septic shock, what level of glucose should trigger one to start an insulin infusion (> 180 or $>150 \mathrm{mg} / \mathrm{dl})$ ?

We identified a recent network meta-analysis of 35 RCTs [519]. The analysis compared four different blood glucose targets $(<110,110-144,144-180$, and $>180 \mathrm{mg} /$ $\mathrm{dL})$. No significant difference in the risk of hospital mortality was observed between the four blood glucose ranges. Target concentrations of $<110$ and $110-$ $144 \mathrm{mg} / \mathrm{dL}$ were associated with a four to nine-fold increase in the risk of hypoglycemia compared with 144-180 and $>180 \mathrm{mg} / \mathrm{dL}$. No significant difference in the risk of hypoglycemia comparing a target of 144-180 and $>180 \mathrm{mg} / \mathrm{dL}$ was demonstrated (OR 1.72; 95\% CI $0.79-3.7)$.

The overall quality of evidence was rated as moderate (Supplementary Appendix 5). Overall, the balance of effects favoured initiation of insulin therapy at a glucose level of $>180 \mathrm{mg} / \mathrm{dl}$. This was principally driven by the increased risk of hypoglycemia observed with lower targets. No significant differences existed between the 
two-insulin initiation blood glucose levels evaluated. After considering the resources required, cost, health equity issues, and applicability to low- and middleincome economies, the panel made a strong recommendation for the initiation of insulin therapy at a glucose level of $\geq 180 \mathrm{mg} / \mathrm{dL}(10 \mathrm{mmol} / \mathrm{L})$.

Further research is indicated to: (1) identify which technologies including electronic glucose management, continuous glucose monitoring, and closed loop systems, can more safely achieve better glycemic control and lower rates of hypoglycemia; and (2) determine the optimal glycemic control for different patient populations including diabetic and nondiabetic patients, medical and surgical patients.

\section{Vitamin C}

\section{Recommendation}

70. For adults with sepsis or septic shock, we suggest against using IV vitamin C

Weak recommendation, low quality of evidence

\section{Rationale}

Vitamin $C$ is known to have anti-inflammatory properties [520]. In 2017, a single centre before and after study reported shorter duration of vasopressor therapy and lower mortality following the administration of combination of high dose vitamin $\mathrm{C}$, hydrocortisone, and thiamine to patients with sepsis and septic shock [521]. Our literature review found one systematic review and metaanalysis [522] (containing six RCTs) and one additional RCT [523].

Our updated analysis (Supplementary Appendix 5) included seven RCTs (416 critically ill patients). The use of vitamin $C$ did not reduce mortality compared to usual care (RR 0.79; 95\% CI 0.57-1.1, low quality). One study reported reduced vasopressor use at $168 \mathrm{~h}$ [523]. Of the patients alive at 7 days, $22 \%(16 / 72)$ administered vitamin $\mathrm{C}$ remained on vasopressor therapy compared to $10 \%$ $(6 / 59)$ of controls.

Subsequent to the censor date for our literature search, the results of two additional RCTs of Vitamin $\mathrm{C}$ versus placebo were published $[524,525]$. In the study by Fujii et al. [524], 211 adults with septic shock were randomised to the combination of vitamin $\mathrm{C}$, hydrocortisone, and thiamine vs hydrocortisone alone. The authors reported no difference for the primary outcome of time alive and free of vasopressors up to $168 \mathrm{~h}$ between the intervention and control group (median 122.1 h [IQR 76.3-145.4 h] vs
124.6 h [IQR 82.1-147 h]; $p=0.83)$. Ninety-day mortality was $28.6 \%(30 / 105)$ in the vitamin C group, and $24.5 \%$ (25/102) in the control group (HR 1.18; 95\% CI 0.69-2.0). In the study by Moskowitz et al. [525], 200 patients were randomised to a combination of vitamin $\mathrm{C}$, hydrocortisone and thiamine vs placebo. No difference in the primary outcome of mean SOFA score change at $72 \mathrm{~h}$ post enrolment was observed. At 30 days, 34.7\% (35/101) of patients randomised to combination therapy had died vs. $29.3 \%(29 / 99)$ randomised to placebo (HR, 1.3; 95\% CI $0.8-2.2 ; p=0.26)$. When these data are added to our meta-analysis, the point estimate for mortality becomes RR 0.9 (95\% CI 0.69-1.18: low quality).

The overall size of any desirable effect was judged as small with a low quality of evidence (Supplementary Appendix 5). There are limited available data of any undesirable effects: it was noted that the point estimate of the HR for 90-day mortality in the largest RCT [524] was 1.18 (95\% CI $0.69-2.00)$ i.e. favouring the control group. The balance of effects was accordingly judged as favouring neither the intervention nor the comparator. The intervention itself requires limited resources and is feasible in low- and middle-income economies.

The panel issued a weak recommendation against the use of vitamin $C$ in patients with sepsis and septic shock. The results of ongoing RCTs may influence the quality of evidence and future updates of the guidelines.

\section{Bicarbonate therapy \\ Recommendations \\ 71. For adults with septic shock and hypoperfusion-induced lactic acidemia, we suggest against using sodium bicarbonate therapy to improve haemodynamics or to reduce vasopressor requirements \\ Weak recommendation, low quality of evidence \\ 72. For adults with septic shock, severe metabolic acidemia $(\mathrm{pH} \leq 7.2)$ and AKI (AKIN score 2 or 3 ), we suggest using sodium bicarbonate therapy \\ Weak recommendation, low quality of evidence}

\section{Rationale}

The previous guidance was based on two small, blinded crossover RCTs that compared equimolar saline vs sodium bicarbonate in patients with lactic acidosis and failed to reveal any difference in haemodynamic variables or vasopressor requirements [526, 527]. A weak recommendation was made against the use of bicarbonate therapy to improve haemodynamics or to reduce vasopressor requirements in patients with hypoperfusion-induced lactic acidemia with $\mathrm{pH} \geq 7.15$. 
Our literature search identified one new RCT [528]. In this multicentre trial, 400 patients with severe metabolic acidemia $(\mathrm{pH} \leq 7.20)$ were randomly allocated to receive IV $4.2 \%$ sodium bicarbonate with the aim of achieving an arterial $\mathrm{pH}$ of 7.3, or control (no bicarbonate). No between-group difference was observed in the primary outcome of a composite of 28-day mortality and organ failure at day 7 . However, hypernatremia, hypocalcaemia, and metabolic alkalosis were observed more frequently in those randomised to bicarbonate. In the subgroup of patients with AKI defined as AKI Network (AKIN) stage 2 or 3 at randomisation (182/389-47\%), lower mortality was observed with bicarbonate therapy: control 57/90 (63\%), bicarbonate $(42 / 92-46 \%)$, absolute risk reduction (ARR) $-17.7 \%$ ( -33.0 to -2.3$), p=0.016$. There was a significant differential effect between patients with an AKIN score of 2 or 3 compared with those with a score of $0-1$ ( $\mathrm{p}$ value for heterogeneity $=0.023$ ).

Sepsis was present in $61 \%(238 / 389)$ of patients at the time of randomisation. No differential effect was observed between patients with vs without sepsis. The outcomes of patients with both sepsis and AKI were not reported.

Overall, the quality of evidence is low (Supplementary Appendix 5). The summary of judgements supported a weak recommendation against the intervention. The 2016 recommendation is essentially unchanged. However, when considering the subset of patients with septic shock, severe metabolic acidosis and AKI, the balance of effects probably favours IV bicarbonate. A weak recommendation for the use of IV bicarbonate in this population was made.

\section{Nutrition}

Recommendation

73. For adult patients with sepsis or septic shock who can be fed enterally, we suggest early (within 72 h) initiation of enteral nutrition Weak recommendation; very low quality of evidence

\section{Rationale}

The early administration of enteral nutrition in patients with sepsis and septic shock has potential physiologic advantages related to the maintenance of gut integrity and prevention of intestinal permeability, dampening of the inflammatory response, and modulation of metabolic responses that may reduce insulin resistance $[529,530]$. Our literature search defined early enteral nutrition as enteral nutrition commenced within $72 \mathrm{~h}$ of ICU admission. The comparator was enteral nutrition commenced after $72 \mathrm{~h}$.

The literature search identified one new RCT [531]. This multicentre trial conducted in 44 French ICUs randomised
2410 invasively mechanically ventilated patients with shock to early enteral nutrition vs early parenteral nutrition. 1504 (62\%) of the participants had sepsis. The results of this trial were included in a meta-analysis with four relevant trials from the 2016 guidelines [532-535]. No significant effect favouring early enteral nutrition was observed for all outcomes evaluated. The quality of evidence was assessed low or very low: downgrades were for risk of bias, inconsistency, and imprecision.

The overall balance of effects did not favour either early enteral feeding (within $72 \mathrm{~h}$ ) compared with enteral feeding commenced after that time. Although the available evidence is of low quality, it does not suggest harm following the institution of early enteral feeding. Neither intervention was considered more beneficial when considering resources utilisation, cost effectiveness, and equity issues. The institution of early enteral nutrition was also considered feasible in low- and middle-income economies.

Given the plausible possibility of benefit when considering the available physiological data, and the absence of any apparent harm, a weak recommendation to start feeding early in patients with sepsis and septic shock was made. Further research addressing this question in patients with sepsis and septic shock is required.

\section{Long-term outcomes and goals of care}

Patients who survive a protracted period of ICU care for sepsis typically face a long and complicated road to recovery. There will not only be physical rehabilitation challenges to overcome but also great uncertainty about the way to organize and coordinate care, both to promote recovery/avoid complications/recurrence and to ensure care is matched to patient and family goals of care.

There is broad consensus that the current healthcare system is likely falling short of what optimal care during the recovery period might look like for this patient population. However, generating a robust evidence base upon which to make concrete recommendations about changes in the care paradigm has proven to be extraordinarily difficult. Some of the difficulties relate to:

- not all patients are the same, and understanding which patients ought to receive which interventions is very poor;

- not all healthcare delivery systems are the sameeven within one system, some patients may be very well-supported while others may not-really complicating what 'control' care looks like;

- lack of understanding about dosing and intensity of many of the proposed interventions, and when and whether they should be combined in packages is generally missing. 
While these issues of patient heterogeneity, variable control care, and lack of understanding about ideal configuration of interventions are protean, they are exquisitely true in this setting: while two ICUs may be different, each ICU discharges patients into a broad and variable milieu of settings. The variation in both ICU and post-ICU management of critically ill patients increases the complexity of understanding and defining best practice.

Thus, putting all this together, there are some overarching conceptual features about 'best practice' that the panel endorses, recognising, however, that the nature, timing, and combination of these broad aspects of care may vary, and strong unambiguous evidence for the 'how to' for these things is often going to be lacking.

\section{Goals of care}

Recommendations

74. For adults with sepsis or septic shock, we recommend discussing goals of care and prognosis with patients and families over no such discussion

Best Practice Statement

75. For adults with sepsis or septic shock, we suggest addressing goals of care early (within $72 \mathrm{~h}$ ) over late [72]

Weak recommendation, low-quality evidence

76. There is insufficient evidence to make a recommendation for any specific standardised criterion to trigger goals of care discussion

\section{Rationale}

Patients with sepsis or septic shock are at high risk of multi-organ failure, long-term functional sequelae, and death. Some patients may accept any and all treatment for their condition, but others may consider limitations depending on prognosis, invasiveness of interventions, and predicted quality of life (QoL). A discussion of goals of care and prognosis is essential to determine which treatments are acceptable and those interventions that are not desired [536].

No studies were identified that compared discussions of goals of care and prognosis versus no such discussion in critically ill or septic patients. While advance care planning in patients with life-limiting illness may reduce use of life-sustaining treatments, it may also increase use of hospice and palliative care, and improve concordance between treatment and patient values [537]. The relevance of advance care planning for future health needs to goals of care discussions at the time of a critical illness is unclear. Despite lack of evidence, the panel recognised that discussion of prognosis and exploration of goals of care with patients and/or family is a necessary precondition to determine patient treatment preferences and providing value-concordant care. Thus, the panel made a best practice recommendation to discuss goals of care and prognosis with patients and families.

The timing of discussions of goals of care and prognosis in the ICU was addressed in one study where $26 \%$ of patients had infection or sepsis as a primary diagnosis [538]. A multicomponent family support intervention included a meeting at $48 \mathrm{~h}$ after ICU admission that included discussion of goals of care and prognosis. The support intervention did not affect family psychological outcomes but did improve perceived quality of communication and perception of patient- and family-centeredness of care. A reduction in ICU length of stay was noted, yet it is unknown if the reduction is due to increased mortality. Based on this study, early (within $72 \mathrm{~h}$ of ICU admission) discussion of the goals of care is suggested.

We identified several studies exploring the use of specific criteria to trigger a goals of care discussion in critically ill patients, though none report the proportion of patients with sepsis or septic shock. Conflict over valuesbased treatment was used to trigger ethics consultation in the intervention group in three randomised ICU studies [539-541]. Reductions in ICU and ventilator days in intervention patients who died before hospital discharge were found in two studies $[539,540]$, and the third study found overall shorter ICU and hospital stay in the ethics consultation group [541]. Ethics consultation did not affect overall mortality in any study. Duration of mechanical ventilation and duration of ICU stay were used to trigger specific interventions in two randomised studies [542, 543]. The study by Carson et al. randomised patients after 7 days of mechanical ventilation to a group receiving an informational brochure and two family meetings with palliative care specialists to address goals of care or a group receiving an informational brochure and meetings led by the ICU team [543]. Palliative care meetings failed to show benefit in decreasing anxiety and depression in surrogate decision makers in the intervention group but did increase post-traumatic stress disorder (PTSD) symptoms. No benefit was demonstrated on family satisfaction, ICU days, or hospital days. Andereck et al. randomised patients after 5 days or more in a medical-surgical ICU to proactive ethics consultation versus usual care [542]. Ethics consultation did not result in a reduction in ICU stay, hospital stay, or life-sustaining treatments in patients who did not survive to discharge. Neither study demonstrated an effect of interventions on mortality. One study [544] investigated the use of an automated early warning system alert in patients hospitalised on medical units $(27 \%$ with infection). The early warning system did not impact hospital mortality or hospital length of stay, but did reduce ICU transfers and ICU length of stay and increased documentation of advance directives and resuscitation status compared to the usual care group. 
Given the variety of triggers used in these studies and the lack of superiority of any single trigger, no recommendation can be made for specific criteria to initiate a goals of care discussion. The timing of and triggers for such discussions should take into account the current condition of the patient, premorbid health and QoL, prognosis, response to treatment, interventions under consideration, anticipated QoL following treatment, availability of resources, and readiness and ability of the patient or family to engage in the discussion.

Public members judged it important to assess patient and family understanding of the information provided in goals of care discussion and for a member of the care team to check with them to determine if further explanations are needed. Additional input included the recommendation that a goals of care discussion should take into account chronic medical conditions in addition to sepsis.

\section{Palliative care}

\section{Recommendations}

77. For adults with sepsis or septic shock, we recommend integrating principles of palliative care (which may include palliative care consultation based on clinician judgement) into the treatment plan, when appropriate, to address patient and family symptoms and suffering Best Practice Statement

78. For adults with sepsis or septic shock, we suggest against routine formal palliative care consultation for all patients over palliative care consultation based on clinician judgement

Weak recommendation, low-quality evidence

\section{Rationale}

While the goal of treating most patients with sepsis or septic shock is to improve survival, some patients have significant comorbidities that may be life limiting or significantly impair QoL. Palliative (supportive) care may be particularly helpful in patients with sepsis who are not responding to treatment or for whom sepsis is an endstage manifestation of their underlying chronic illness. Studies have evaluated palliative care interventions in the ICU but not specifically in patients with sepsis [543, 545548]. However, indirect evidence from these studies was judged likely to apply to patients with sepsis.

Criteria for patient inclusion and the interventions in these studies demonstrate significant heterogeneity. Inclusion criteria for ICU patients consisted of mechanical ventilation for 7 days [543], high risk on a palliative care screen [548], physician determination that care should not be escalated or care should be withdrawn [545], physician belief that the patient would die in a few days [547], or death in the ICU or within $30 \mathrm{~h}$ of transfer out of the ICU [546]. Interventions comprised formal palliative care consultation [543, 545, 548], a complex quality improvement project to improve end-of-life care
[546], and a planned end-of-life conference conducted by intensivists according to specific guidelines along with a bereavement brochure [547].

Various outcome measures are reported but none of the studies evaluated critical patient-centered outcomes such as QoL, physical or cognitive recovery, psychological outcomes, or symptoms. Only one study with a structured palliative care intervention [547] demonstrated a beneficial effect of lower prevalence of anxiety and depression symptoms and PTSD symptoms in family members 90 days after the patient's death. In contrast, Carson et al. found an increase in PTSD symptoms in family surrogate decision makers with palliative care consultation [543]. Palliative care interventions had no significant impact on family satisfaction with care, ICU length of stay [543, $545-548]$, hospital length of stay [543, 545, 548], or mortality [543, 545, 548].

Overall evidence for routine formal palliative care interventions in ICU patients is of low quality and provides mixed evidence of benefit. Thus, the panel suggests against routine formal palliative care consultation for all patients with sepsis or septic shock, instead using clinician judgment to determine which patients and families may benefit from a palliative care consultation.

Despite the lack of evidence for formal palliative care consultation, the panel and public members judged that the principles of palliative care, whether instituted by palliative care specialists, intensivists or other clinicians are essential to address symptoms and suffering in patients and their families. Therefore, the panel made a best practice statement recommending incorporation of palliative care principles in the care of patients with sepsis and septic shock.

\section{Peer support groups}

\section{Recommendation}

79. For adult survivors of sepsis or septic shock and their families, we suggest referral to peer support groups over no such referral

Weak recommendation, very low quality of evidence

\section{Rationale}

Peer support groups have been used to enhance recovery from illness when survivors have long-lasting disability but have only recently been used in critical care and sepsis [549-551]. With increased recognition of post-intensive care syndrome (PICS) in survivors of critical illness and their families, peer support represents a patient-centered approach to improve long-term outcomes [552, 553]. Public members suggested that referral to an individual peer support person during the sepsis hospitalisation may provide a means of support and hope for recovery while referring sepsis survivors and their 
families to a peer support group may help them regain functional and emotional health.

Models of peer support are numerous and include community-based in person or virtual peer support; outpatient ICU follow-up clinics (with or without psychologist support); within-ICU peer support; and individual peer mentors [551]. We did not identify sufficient studies to allow for meta-analysis. Four observational studies examined the impact of peer support groups on ICU patients, though they were not specific to sepsis patients. These studies evaluated the impact of peer support in ICU survivors from a surgical ICU [554], two general ICUs [555-557] and two cardiac ICUs [555, 558]. Group models varied, with facilitated in-person [554, 557], group-based integrated with rehabilitation $[555,556]$ or a "buddy" with a former patient-to-patient programme [558]. In several qualitative studies, ICU survivors described peer support as a helpful aid to recovery [559-563]. Three qualitative studies identified two common themes of peer support, (1) benefit of knowing that others shared similar experiences and (2) benefit of shared coping with others [564].

Overall quality of evidence was judged to be very low for the impact of peer support groups on outcomes. No studies described costs associated with support groups, which will vary given the model and resource availability. Research evaluating support groups is needed with at least two RCTs planned [564-566].

Despite the very low certainty of evidence, the panel made a weak recommendation in favour of referring patients and families to peer support, which will increase the equity of access to such services. As individuals who receive referral to peer support have the choice to participate or not (based on personal preference, timing, location, functional status, and resources required) a weak recommendation provides an opportunity to access support for sepsis survivors who otherwise may not know where to turn [552].

\section{Transitions of care}

\section{Recommendations}

80. For adults with sepsis or septic shock, we suggest using a handoff process of critically important information at transitions of care, over no such handoff process

Weak recommendation, very low-quality evidence

81. There is insufficient evidence to make a recommendation for the use of any specific structured handoff tool over usual handoff processes

\section{Rationale}

Transitions of care are prone to communication errors, which have been identified as a barrier to the timely detection and management of sepsis [567]. Improving handoff at transitions in care represents an opportunity to improve patient outcomes across the entire spectrum of sepsis care, from hospitalisation to return to the community.

We did not identify any studies specifically evaluating patients with sepsis. Structured handoff interventions for critically ill patients have been evaluated at many transitions of patient care (ED/ICU, OR/ICU, ICU/ward, and hospital/home). The majority are observational pre-post studies and report process measures such as completeness and accuracy of communication rather than clinical outcomes. There were insufficient data to allow for meta-analysis.

A single RCT using a stepped-wedge design in 8 ICUs evaluated the impact of a standardised handoff process, finding no effect upon duration of mechanical ventilation, ICU length of stay or duration of handover [568]. Observational studies of structured handoff process have demonstrated mixed effects, with some finding reductions in unexpected clinical events [569], or ICU readmission $[570,571]$ and others without impact upon length of stay [572], mortality $[572,573]$ or hospital readmission [572, 573].

Overall quality of evidence was judged to be very low. While it is unclear whether structured handoffs impact important patient outcomes, many sepsis interventions and tests are time-dependent and communication failures may increase the chances of critical medical errors. Structured handoff processes appear to result in more complete and accurate transfer of information, without any undesirable effects. Thus, despite the low certainty of evidence, the panel made a weak recommendation in favour of structured handoff processes at transitions of care. Of the structured handover tools studied, none specifically applies to sepsis. Given the wide variety of hospital staffing models, medical records, and discharge processes, along with the lack of evidence to recommend any one tool over another, the panel chose to make no recommendation for a specific structured handover tool.

\section{Screening for economic or social support}

Recommendation

82. For adults with sepsis or septic shock and their families, we recommend screening for economic and social support (including housing, nutritional, financial, and spiritual support), and make referrals where available to meet these needs

Best Practice Statement

\section{Rationale}

Non-medical social needs and potentially modifiable factors such as economic and social support largely influence health outcomes. While survival from sepsis is 
improving, long-term health requires survivors to have the resources to recover and thrive. Notably, critically ill patients have a decline in socio-economic status (SES) after their illness [574]. Many observational studies describe the relationship between various socioeconomic supports and patient outcomes that suggest that low SES, substance abuse and poor nutritional status lead to poor outcomes, and that critical illness itself results in lower SES post-illness. Additionally, living in neighborhoods with low SES is associated with an increased risk of sepsis [575], community-acquired bacteremia [575] and death from bacteremia [576] and worse outcomes [577]. Racial disparities in sepsis [578] are at least partially explained by living in medically underserved neighborhoods [579].

Screening for economic and social support may help reduce these inequities. Although socioeconomic screening is considered part of standard clinical practice, all clinical teams in many settings may not do it. This may be particularly true in the critical care setting where patients are often unable to communicate, and social determinants of health may not be addressed during management of the acute illness.

No studies were identified comparing screening versus no screening for economic and social support. Furthermore, it is unlikely that many research studies would be conducted, since locally available social needs and supports vary. In LMIC where resources are limited, needs may be vast. Despite these variations, social and economic screening may identify challenges that sepsis survivors are experiencing, allowing clinicians to identify potential resources and referrals, which can assist to improve long-term health outcomes.

\section{Sepsis education for patients and families}

Recommendation
83. For adults with sepsis or septic shock and their families, we suggest
offering written and verbal sepsis education (diagnosis, treatment, and
post-ICU/post-sepsis syndrome) prior to hospital discharge and in the
follow-up setting
Weak recommendation, very low-quality evidence

\section{Rationale}

Almost 40\% of sepsis survivors are re-hospitalised within 3 months, often for preventable conditions [580], contributing to increased healthcare costs [581]. Given the risk of post-sepsis morbidity, sepsis education may have a role in the timely healthcare seeking behavior in sepsis survivors who experience complications. In an international survey of sepsis survivors from 41 countries, $45 \%$ and $63 \%$ reported dissatisfaction with sepsis education at the acute and post-acute phase, respectively
[582]. We identified six RCTs that evaluated educational interventions for critically ill patients and their families [583-588]. Only one specifically studied patients with sepsis [588], evaluating a complex intervention, which included education along with primary care follow-up and post-discharge monitoring. Varied education methods were employed, including delivery by trained nurses [586, 588], multimedia nursing education [585], information booklets developed by nurses [584], a family information leaflet [583], and informational videos with accompanying web-based content [587].

These studies provided limited data for review. ICU education did not appear to impact patients' anxiety and depression [584, 586, 588], but did improve families' satisfaction with care [583]. The panel judged that education would likely have variable acceptability, as a qualitative study showed that patients who survived sepsis had diverse viewpoints ranging from appreciating the education about sepsis to not being able to recall the education session, to even disliking it as a reminder of the severity of their condition [587]. Based on these data and feedback from the public panel, we suggest that multiple opportunities for education be offered prior to hospital discharge and in the follow-up setting, taking into account patients' and/or families' readiness to process information. Sepsis education is regarded as a low cost intervention and feasible, even in low-resource settings, as a number of online and published sepsis education resources exist [589]. Future studies are needed to better understand the effects, the cost-effectiveness, and the optimal approach for educating patients and families after sepsis.

\section{Shared decision making}

\section{Recommendation}

84. For adults with sepsis or septic shock and their families, we recommend the clinical team provide the opportunity to participate in

shared decision making in post-ICU and hospital discharge planning to ensure discharge plans are acceptable and feasible

Best Practice Statement

\section{Rationale}

Shared decision making (SDM) is a process in which health professionals, patients and their caregivers collaborate in making decisions about a patient's care options [590]. This patient-centered approach may be less routinely used in post-ICU and hospital discharge planning than in other aspects of acute patient care. No studies were identified that compared SDM with other types of ICU or hospital discharge planning. Despite the lack of evidence, SDM in discharge planning as in other care decisions is more likely to result in decisions consistent 
with the values and preferences of the patient and family. Patient and family involvement in discharge planning may also increase family satisfaction. A small study of ICU relatives found that anxiety and depression rates were lower in those who preferred an active role or shared responsibility in decision-making compared to those who preferred a passive role [591]. A family care conference with nursing staff at the time of discharge from the ICU resulted in lower anxiety scores for family members compared to a control group although it is not clear that families participated in SDM [592]. Family caregivers of critically ill patients discharged home felt overwhelmed and unprepared and had difficulty managing expectations [593]. Communication through SDM at the time of ICU or hospital discharge may improve support for family caregivers as communication was found to be important to decision-making for family surrogates of chronic critically ill patients [594]. Studies of tools employed to promote SDM in patients with other serious illnesses show improved patient knowledge and awareness of treatment options [595]. Due to the potential benefits of SDM and the current emphasis on patientcentered care, the opportunity for patients and/or family to participate in SDM for ICU and hospital discharge planning is recommended as a best practice statement.

\section{Discharge planning}

\section{Recommendations}

85. For adults with sepsis and septic shock and their families, we suggest using a critical care transition programme, compared to usual care, upon transfer to the floor

Weak recommendation, very low-quality evidence

86. For adults with sepsis and septic shock, we recommend reconciling medications at both ICU and hospital discharge

Best Practice Statement

87. For adult survivors of sepsis and septic shock and their families, we recommend including information about the ICU stay, sepsis and related diagnoses, treatments, and common impairments after sepsis in the written and verbal hospital discharge summary Best Practice Statement

\section{Rationale}

Transfer from ICU to general floor and discharge from the hospital are both vulnerable periods for patients, with high frequency of medication errors and information loss [596-602]. Sepsis patients, with longer than average hospitalisations and higher comorbidity burden, may be at particular risk for poor outcomes with transitions. Several studies, mostly before-and-after design, have examined the impact of critical care transition programmes on reducing ICU readmission or death among patients transferred from ICU to the ward [597, 601, 603-611]. These programmes have used varied models, but generally involve ICU clinicians (e.g., nurse, respiratory therapist, and/or physician) following patients daily on the wards after transferring out of the ICU for a few days or until clinically stable. Meta-analysis of these studies suggests that critical care transition programmes reduce risk of in-hospital mortality and potentially reduce risk of ICU readmission. Effects on ICU workload and workflow have not been systematically examined. Public panel members were supportive of such programmes, as they may provide reassurance and a sense of protection to patients after they leave the ICU.

Medication reconciliation is broadly recognised to be important during patient transitions. Hospitalisation and ICU admission are high-risk periods for unintentional medication error-both continuations of medications for temporary indications and unintentional discontinuations of chronic medications [596, 599, 600, 602]. Medication reconciliation has been associated with fewer medication errors $[598,612]$ and may help reduce hospital readmission $[613,614]$. Given the frequency of medication changes during an ICU stay, we recommend reconciling medications at both ICU and hospital discharge. Medication reconciliation surrounding sepsis hospitalisation involves getting the correct list of medications and adjusting medication dosing regularly in response to dynamic physiologic changes during and after critical illness [580].

Key information from hospitalisation is often missing on hospital discharge documentation [615-618]. Information on post-intensive care syndrome (PICS) may be provided to only one in three ICU survivors [550, 618], mechanical ventilation, dialysis), and common impairments after sepsis. We recommend providing information about the ICU stay, sepsis diagnosis, key treatments (e.g. mechanical ventilation, dialysis), and post-ICU/ post-sepsis syndrome." to replace sentence fragment "mechanical ventilation, dialysis) and common impairments after sepsis. Public panel members stressed the importance of providing information in both verbal and written form and assessing that the information was understood. There are a growing number of online resources and informational brochures regarding "post intensive care" / "post-sepsis syndrome" [580], but more research is needed to determine the optimal approaches to providing anticipatory guidance to patients and families after critical illness [582, 619]. 
Recommendations

88. For adults with sepsis or septic shock who developed new impairments, we recommend hospital discharge plans include follow-up with clinicians able to support and manage new and long-term sequelae

Best Practice Statement

89. There is insufficient evidence to make a recommendation on early post-hospital discharge follow-up compared to routine posthospital discharge follow-up

\section{Rationale}

Many sepsis survivors experience short and/or long-term sequela such as cognitive and/or physical disability, with ongoing recovery persisting for months to years [620]. Public panelists rated cognitive and physical recovery, psychologic symptoms in survivors and their families, QoL and readmission to the hospital and/or ICU as critically important outcomes. These outcomes were consistent with a 2019 qualitative analysis of health related QoL domains identified by sepsis survivors [621]. Follow-up with a provider after hospital discharge is one-step in the recovery process.

Sepsis survivors are at risk for hospital readmission, which has been associated with increased mortality or discharge to hospice [622, 623]. Hospital readmission within 90 days of discharge occurs in approximately $40 \%$ of sepsis survivors and is associated with high costs [624]. In addition, sepsis survivors are at increased risk for recurrent infection, AKI and new cardiovascular events compared to patients hospitalised for other diagnoses [580]. Observational studies in patients with congestive heart failure have associated early (within 7-14 days) post-discharge follow-up with reduced hospital readmissions [625]. Among older adults, early post discharge follow-up (within 7 days) with a primary care physician was associated with lower risk of 30-day readmission [626, 627].

Three studies, one RCT [628] and two observational studies $[629,630]$ evaluated early post-hospital follow-up in patients with critical illness. None of the three studies specifically evaluated a sepsis population or reported the proportion of sepsis patients. The interventions and QoL measures varied among the three studies each with severe limitations. In an analysis of older adults with severe sepsis, one study found that the combination of early home health care and a visit with a medical provider was associated with a reduced readmission risk [631]. There were insufficient studies to allow meta-analysis and the limited evidence is of very low quality.

Despite these limitations, the panel recommends follow-up with a provider after hospital discharge to manage new impairments associated with sepsis. Due to the low quality and lack of evidence specific to sepsis, we are unable to make a recommendation for early (7-14 days) provider follow-up versus routine follow-up upon hospital discharge. Timely, coordinated resources and provider follow-up may lead to improved QoL for sepsis survivors, however further research on the impact of post-discharge follow-up is needed.

\section{Cognitive therapy}

\section{Recommendation}

90. There is insufficient evidence to make a recommendation on early cognitive therapy for adult survivors of sepsis or septic shock

\section{Rationale}

Sepsis is associated with newly acquired cognitive impairment and functional disability amongst survivors [620]. Long-term impairments in memory, attention, verbal fluency, decision-making and executive functioning may be linked to a variety of mechanisms such as metabolic derangements, cerebral ischaemia, overwhelming inflammation, disrupted blood-brain barrier, oxidative stress, and severe microglial activation, particularly within the limbic system [632]. A feasibility, pilot, randomised trial in general medical/surgical ICU survivors comparing usual care to an intervention of combined in-home cognitive, physical, and functional rehabilitation following discharge showed improved executive functioning at 3 months [633]. Some small single centre studies tested specific early cognitive therapies to enhance cognitive and overall functional recovery after critical illness [634, 635].

A proof-of-concept single-centre pilot study aimed to evaluate the efficacy and safety of the use of a multifaceted early intervention (cognitive therapy within ICU) in patients with respiratory failure and/or shock [634]. ICU patients were randomised to receive either combined cognitive and physical therapy or physical therapy alone. The results demonstrated that the intervention was feasible and safe, but the study was underpowered and therefore inconclusive regarding its clinical effects on cognitive function and health-related QoL outcomes at 3-month follow-up. In addition, a prospective cohort study testing a series of cognitive training sessions starting in the ICU and continued for up to 2 months, found overall minimal clinical relevance as Minimum Clinically Important Difference (MID) of Montreal Cognitive Assessment (MOCA) was small, with some meaningful results in younger patients, but not in the middle-aged or older population $[635,636]$.

In view of these findings, the panel judged there to be insufficient evidence to make a recommendation. In centers where cognitive therapy is used, it could reasonably be continued as it is likely acceptable and feasible, 
but there is insufficient evidence to change practice in centers without such therapy. Further larger studies in patients with sepsis are required to determine the impact of early cognitive therapy, as well as costs and type of intervention.

\section{Post-discharge follow-up}

Recommendations

91. For adult survivors of sepsis or septic shock, we recommend assessment and follow-up for physical, cognitive, and emotional problems after hospital discharge

Best Practice Statement

92. For adult survivors of sepsis or septic shock, we suggest referral to a post-critical illness follow-up programme if available

Weak recommendation, very low-quality evidence

93. For adult survivors of sepsis or septic shock receiving mechanical ventilation for $>48 \mathrm{~h}$ or an ICU stay of $>72 \mathrm{~h}$, we suggest referral to a post-hospital rehabilitation programme

Weak recommendation, very low-quality evidence

\section{Rationale}

Given the prevalence of new or worsening physical, cognitive, and emotional problems experienced by sepsis survivors [580,620], we recommend assessment and follow-up for these problems after hospital discharge. There are insufficient data to suggest any specific tool to assess for these problems, and the optimal approach will vary by patient and setting. At a minimum, physicians should ask patients and families about new problems in these domains.

Post-critical illness programmes have been developed as a means of screening for and addressing the multi-faceted issues faced by ICU survivors. These programmes vary in their structure, and are not consistently available worldwide [637]. Few randomised studies have assessed post-critical illness clinics [588, 628, 638, 639], and-consistent with a recent Cochrane review [640]our meta-analysis found no differences from usual care in terms of mortality, QoL, physical function, or cognition, with possible small improvements in psychological symptoms (anxiety, depression, PTSD). More studies of post-sepsis follow-up programmes are in process [641, 642]. We suggest offering referral to post-critical illness clinics where available. While efficacy data are equivocal, these programmes are consistently well-liked by patients and offer an environment to learn about challenges sepsis survivors face, as well as to pilot and test interventions for enhancing recovery [637, 643]. Lessons learned in post-critical care clinics could be adapted to other, morescalable interventions such as telehealth.

Several randomised studies have assessed physical rehabilitation programmes for survivors of critical illness [581, 606, 644-651]. These studies focused on critically ill patients, generally defined by days in ICU or days with mechanical ventilation and begin on the floor or post-hospital setting. Meta-analysis suggests possible small improvements in QoL and depressive symptoms, but no difference in mortality, physical function, or anxiety. Nonetheless, based on their strong rationale, and benefit in related populations [580] (e.g., older patients with cognitive impairment, patients following stroke or traumatic brain injury), we suggest referral to rehabilitation programmes in survivors of sepsis. This suggestion is consistent with the guidance of several expert panels [646, 652, 653]. Future research is needed to determine an optimal approach to functional rehabilitation (timing, dosing, intensity, duration) and patient selection [643].

\section{Supplementary Information}

The online version contains supplementary material available at https://doi. org/10.1007/s00134-021-06506-y.

\section{Electronic supplementary material}

- Methodology

- Appendix 1. Screening and Early Treatment

- Appendix 2. Infection

- Appendix 3. Haemodynamic Management

- Appendix 4. Ventilation

- Appendix 5. Additional Therapies

- Appendix 6. Goals and Long Term Outcomes

- Appendix 7. Search Strategy

\section{Author details}

${ }^{1}$ Division of Pulmonary, Critical Care and Sleep Medicine, University of Washington, Seattle, WA, USA. ${ }^{2}$ Adult Critical Care, St George's University Hospitals NHS Foundation Trust \& St George's University of London, London, UK.

${ }^{3}$ Department of Health Research Methods, Evidence, and Impact, McMaster University, Hamilton, ON, Canada. ${ }^{4}$ Dipartimento di Scienze dell'Emergenza, Anestesiologiche e della Rianimazione, Policlinico Universitario A. Gemelli IRCCS, Rome, Italy. ${ }^{5}$ Emory University School of Medicine, Atlanta, GA, USA. ${ }^{6}$ Western Health, Melbourne, Australia. ${ }^{7}$ Anesthesiology, Pain and Intensive Care Department, Federal University of São Paulo, Hospital of São Paulo, São Paulo, Brazil. ${ }^{8}$ Ottawa Hospital, Ottawa, ON, Canada. ${ }^{9}$ Guy's \& St Thomas' Hospital, London, England, UK. ${ }^{10}$ University of Michigan and VA Center for Clinical Management Research, Ann Arbor, MI, USA. ${ }^{11}$ Cooper Health System, Camden, NJ, USA. ${ }^{12}$ University of Kansas Medical Center, Kansas City, KS, USA. ${ }^{13}$ ESCMID Study Group for Bloodstream Infections, Endocarditis and Sepsis, Division of Infectious Diseases, Amsterdam UMC, University of Amsterdam, Amsterdam, The Netherlands. ${ }^{14}$ Department of Internal Medicine, College of Medicine and Health Sciences, Emirates University, Al Ain, United Arab Emirates. ${ }^{15}$ University of Pittsburgh Critical Care Medicine CRISMA Laboratory, Pittsburgh, PA, USA. ${ }^{16}$ Intensive Care Department, Ministry of National Guard Health Affairs, King Abdullah International Medical Research Center, King Saud Bin Abdulaziz University for Health Sciences, Riyadh, Kingdom of Saudi Arabia. ${ }^{17}$ School of Medicine, University of Sao Paulo, São Paulo, Brazil. ${ }^{18}$ University of Minnesota, Minneapolis, MN, USA.

${ }^{19}$ Population Health Research Institute, Hamilton, ON, Canada. ${ }^{20}$ Mount Sinai Hospital \& University of Toronto (Leslie Dan Faculty of Pharmacy), Toronto, ON, Canada. ${ }^{21}$ Department of Biomedical Sciences, Humanitas University Pieve Emanuele, Milan, Italy. ${ }^{22}$ Department of Anaesthesia and Intensive Care, IRCCS Humanitas Research Hospital, Rozzano, Milan, Italy. ${ }^{23}$ Department of Anesthesia, McMaster University, Hamilton, ON, Canada. ${ }^{24}$ Lexington Veterans Affairs Medical Center/University of Kentucky College of Medicine, Lexington, KY, USA. ${ }^{25}$ Ghent University Hospital, Ghent, Belgium. ${ }^{26}$ The University of Tokyo, Tokyo, Japan. ${ }^{27}$ Medical ICU, Peking Union Medical College Hospital, Beijing, China. ${ }^{28}$ Hospital Interzonal de Agudos San Martin de La Plata, Buenos Aires, Argentina. ${ }^{29}$ Intensive Care Department, Vall d'Hebron University Hospital, Vall d'Hebron Institut de Recerca, Barcelona, Spain. ${ }^{30}$ Prince of Wales Hospital, Hong Kong, China. ${ }^{31}$ Australian and New Zealand Intensive Care Research 
Centre, Monash University, Melbourne, VIC, Australia. ${ }^{32}$ Department of Intensive Care 4131, Copenhagen University Hospital Rigshospitalet, Copenhagen, Denmark. ${ }^{33}$ University of Michigan Health System, Ann Arbor, MI, USA. ${ }^{34}$ Liverpool School of Tropical Medicine, Liverpool, UK. ${ }^{35}$ Vanderbilt University, Nashville, TN, USA. ${ }^{36}$ Department of Medicine, Brigham and Women's Hospital, Boston, MA, USA. ${ }^{37}$ Department of Population Medicine, Harvard Medical School, and Harvard Pilgrim Health Care Institute, Boston, MA, USA. ${ }^{38}$ ASAN Medical Center, University of Ulsan College of Medicine, Seoul, South Korea. 39 University of Manitoba, Winnipeg, MB, Canada. ${ }^{40}$ Makerere University College of Health Sciences, Kampala, Uganda. ${ }^{41}$ Intensive Care Division, Faculdade de Medicina de São José do Rio Preto, São Paulo, Brazil. ${ }^{2}$ Critical Care Medicine Department, NIH Clinical Center, Bethesda, MD, USA. ${ }^{43}$ Alfred Health, Melbourne, Australia. ${ }^{44}$ Mount Sinai Hospital, Toronto, ON, Canada. ${ }^{45}$ Medanta the Medicity, Gurugram, Haryana, India. ${ }^{46}$ Charlotte Maxeke Johannesburg Academic Hospital and Faculty of Health Sciences, University of the Witwatersrand, Johannesburg, South Africa. ${ }^{47}$ New York University School of Medicine, New York, NY, USA. ${ }^{48}$ Washington University School of Medicine, St. Louis, MO, USA. ${ }^{49}$ Faculty of Nursing, University of Alberta, Edmonton, AB, Canada. ${ }^{50}$ Rigshospitalet, Copenhagen, Denmark. ${ }^{51}$ University of Minnesota/Hennepin County Medical Center, Minneapolis, MN, USA. ${ }^{52}$ Faculty of Medicine, University of Queensland Centre for Clinical Research, The University of Queensland, Brisbane, Australia. ${ }^{53}$ Department of Pharmacy, Royal Brisbane and Women's Hospital, Brisbane, Australia. ${ }^{54}$ Department of Intensive Care Medicine, Royal Brisbane and Women's Hospital, Brisbane, Australia. ${ }^{55}$ Division of Anaesthesiology Critical Care Emergency and Pain Medicine, Nîmes University Hospital, University of Montpellier, Nîmes, France. ${ }^{56}$ University of Pennsylvania, Philadelphia, PA, USA. ${ }^{57}$ ChristianaCare, Newark, DE, USA. ${ }^{58}$ Faculty of Medicine, Hebrew University of Jerusalem, Jerusalem, Israel. ${ }^{59}$ Department of Anesthesiology, Critical Care and Pain Medicine, Hadassah Medical Center, Jerusalem, Israel. ${ }^{60}$ Medizinische Hochschule Hannover and German Center of Lung Research (DZL), Hannover, Germany. ${ }^{61}$ World Federation of Intensive and Critical Care, Brussels, Belgium. ${ }^{62}$ Warren Alpert School of Medicine at Brown University, Providence, Rhode Island \& Rhode Island Hospital, Providence, RI, USA.

\section{Acknowledgements}

It is with great appreciation that we acknowledge the public panel members who shared their insights and experiences as patients/relatives and provided advice which was invaluable to informing the updated guideline. Specifically, we thank: Dana Mirman, sepsis survivor \& member of the Board of Directors of Sepsis Alliance, Idelette Nutma and Marie Mach, and three additional public panel members.

\section{Endorsements}

This manuscript has been endorsed by the following societies: European Society of Intensive Care Medicine, Society of Critical Care Medicine, American Association of Critical Care Nurses, American College of Chest Physicians, American College of Emergency Physicians, American Thoracic Society, African Sepsis Alliance, Asia and Pacific Sepsis Alliance, Association De Medicina Intensiva Brasileira, Australian and New Zealand Intensive Care Society, Canadian Critical Care Society, Chinese Society of Critical Care Medicine, Chest, European Respiratory Society, European Society of Clinical Microbiology and Infectious Diseases, Indian Society of Critical Care Medicine, Infectious Diseases Society of North America, Japanese Society of Intensive Care Medicine, Latin American Sepsis Institute, Society for Academic Emergency Medicine, Scandinavian Critical Care Trials Group, Surgical Infection Society, World Federation of Critical Care Nurses, World Federation of Societies of Intensive and Critical Care Medicine.

\section{Governance of surviving sepsis campaign}

SSC Guidelines Committee Co-chairs: Andrew Rhodes, Laura Evans; SSC Guidelines Committee Co vice-chairs: Hallie Prescott, Marlies Ostermann; SSC Guidelines Committee Oversight Group: Andrew Rhodes, Laura Evans, Waleed Alhazzani; SSC Guidelines Committee Group-heads: Craig French, Flavia Machado, Mitchell Levy, Lauralyn McIntyre, Christa Schorr, Steven Simpson, Joost Wiersinga; SSC Conflicts of Interest Co-chairs: Massimo Antonelli, Craig Coopersmith; GUIDE Methodology group: Waleed Alhazzani (chair), Emilie Belley-Cote, Fayez Alshamsi, John Centofanti, Mark Nunnally, Morten Hylander-Moller, Simon Oczkowski.

\section{Declarations}

\section{Conflicts of interest}

Dr. Alhazzani is the Chair of the Guidelines Chapter for the Saudi Critical Care Society and is the chair of the guidelines in intensive care, development and evaluation (GUIDE) Group, McMaster University Canada. Dr. Antonelli received funding from GE, Toray-Estor, Baxter, Pfizer, Orion, Maquet, and Fisher and Paykel; he was on the board of Baxter and Pfizer, and is a member of the executive committee and past president of Società Italiana di Anestesia Rianimazione e Terapia Intensiva (SIAARTI). Dr. French contributed to the ANZICS Guidelines and the National COVID-19 Guidelines. Dr. Machado is a member of the Executive Committee for the Basics Study (for which Baxter provided the drugs and logistics) and AMIB. Dr. McIntyre is a member of the Canadian Critical Care Society and serves on the Surviving Sepsis Campaign Steering Committee. Dr. Ostermann is a council member of the Intensive Care Society UK and member of the Renal Association UK and World Sepsis Alliance. Dr. Prescott is a member of the ATS Critical Care Program Committee. Dr. Simpson is the president-elect and Chair of CHEST, is a member of the board of directors and medical director of Sepsis Alliance, and Chair of the Sepsis Institute Advisory Board. Dr. Wiersinga is a member of ISF, ESCMID, and SWAB. Dr. Angus received funding from Ferring Pharmaceuticals, Inc and ALung Technologies, Inc. Dr. Beale provides consultancy services for Philips Healthcare with his time billed by his institution. Dr. Beilman is the president of the Surgical Infection Society. Dr. Belley-Cote received grants from Roche and Bayer and is a panel member on the Saudi Critical Care Society COVID-19 Thrombosis Guidelines. Dr. Cecconi is a consultant for Edwards Lifesciences, Cheetah Medical, and Directed Systems and is President of the European Society of Intensive Care Medicine. Dr. Coz is a board member of the American College of Chest Physicians. Dr. De Waele consulted for Accelerate, Bayer, Grifols, Pfizer, and MSD with all honoraria paid to Ghent University; he is a Senior Clinical Investigator with the Research Foundation Flanders. Dr. Dellinger serves as an expert witness on occasional medical legal case reviews. Dr. Doi is a member of the Japanese Society of Intensive Care Medicine. Dr. Du is a member of the Chinese Society of Critical Care Medicine and the Chinese College of Intensive Care Medicine. Dr. Ferrer received funding from Grifols, MSD, Pfizer, Shionogi, Toray, Jafron, and Cytosorbents; he is a member of SEMICYUC. Dr. Gomersall is a member of an educational subgroup of the International Forum of Acute Care Trialists. Dr. Hodgson is a member of the Australian National Health and Medical Research Council guidelines (COVID-19) and leading funded trials in early rehabilitation and ECMO. Dr. Møller contributed to guideline work for DASAIM, SSAI, GUIDE, and ESA. Dr. Iwashyna is a member of the ATS, the $\mathrm{NIH}$, and an informal (unincorporated) organization called the Critical and Acute Illness Recovery Organization. Dr. Jacob co-directs the African Research Collaboration on Sepsis (ARCS, funded by UK National Institute for Health Research, sponsored by Liverpool School of Tropical Medicine), he is Secretary General for the African Sepsis Alliance, and is a technical expert for the World Health Organization panels. Dr. Kleinpell is a board member of the World Federation of Intensive and Critical Care, American Nurses Credentialing Center, and the Tennessee Nurses Association Political Action Committee. Dr. Klompas received funding from Up-to-Date; he is a member of the guidelines committees of the Infectious Disease Society of America (IDSA) and Society of Healthcare Epidemiologists of America (SHEA). Dr. Koh is a member of The Korean Society of Critical Care Medicine, The Korean Academy of Tuberculosis and Respiratory Diseases, The Korean Society of Medical Ethics, and the Asia Ventilation Forum. Dr. Kumar served as an expert witness regarding a lethal dose of narcotics. Dr. Kwizera is president of the Intensive Care Society of Uganda and PRO for the Association of Anesthesiologists of Uganda. Dr. Lobo received funding from Pfizer, MSD, Edwards, and Nestle; she is the principal investigator in new antibiotics research led by CROs/industry; she is a member of the AMIB Executive Board and was elected president for 2020-2021. Dr. McGloughlin is a member of ANZICS (Australian New Zealand Intensive Care Society). Dr. Mehta participated in two non-interventional studies by ISCCM-Hermes and Indicaps. Dr. Mer has been an invited speaker for educational talks in industry-sponsored symposia for which honoraria was received; he is the current Vice President of the Southern African Society of Thrombosis and Haemostasis (SASTH), and is involved in annual congress organization; he is an invited author of the Global guidelines for the diagnosis and management of mucormycosis: an initiative of the European Confederation of Medical Mycology in cooperation with the Mycoses Study Group Education and Research Consortium. Dr. Nunnally is the treas- urer of SOCCA, committee 
member of ASA, NYSSA, IARS, AUA, and SAAAPM and serves on the American College of Critical Care Medicine Board of Regents. Dr. Oczkowski is a member of the European Respiratory Society, and contributed to the High Flow Nasal Cannula Guidelines, the Non-Invasive Ventilation in COPD Guidelines. Dr. Osborn received funding from Viven Inc, Inflammatrix, Beckman, and the Foundation for Barnes Jewish Hospital; she is on the advisory board for Beckman, Inflammatix, and Viven; she is a member of the American College of Emergency Physicians, American College of Chest Physicians, American Medical Association, Society of Academic Emergency Medicine, and American Academy of Emergency Physicians; she served as an expert witness in a case related to viral as compared to bacterial sepsis. Dr. Papathanassoglou is a member of the World Federation of Critical Care Nurses (Editor of Journal) and the Canadian Association of Critical Care Nurses. Dr. Perner received a re- search grant from Pfizer Denmark. Dr. Puskarich is the co-inven- tor of a patent to assess LOcarnitine drug responsiveness in sepsis (USPO 10330685); he is a member of the Society for Academic Emergency Medicine, American College of Emergency Physicians (ACEP); he was invited to a recently gathered ACEP early sepsis treatment policy task force asked to develop spe- cialty recommendations for early sepsis treatment. Dr. Roberts received funding from MSD, The Medicines Company, Cardeas Pharma, BiomerieuX, QPEX, Cipla, and Pfizer; he consulted for MSD, QPEX, Discuva Ltd, Accelerate Diagnostics, Bayer, Biomerieux, UptoDate, and Australian Therapeutic Guidelines; he is a member of the Society of Hospital Pharmacists of Australia Leadership Committees for Critical Care and Infectious Diseases and the Lead of Sepsis Working group for the International Society of Anti-infective Chemotherapy. Dr. Schweickert is a paid consultant to the American College of Physicians (last performed in Spring, 2019). Dr. Seckel volunteers for AACN and is a paid consultant to revise online Critical Care Orientation. Dr. Sevransky received funding from the Marcus Foundation- PI VICTAS Trial and serves on the American College of Critical Care Medicine Board of Regents. Dr. Welte received funding from Astellas, AstraZeneca, Boehringer, Basilea, Bayer, Berlin-Chemie, Grifols, Infectopharm, Mundipharma, MSD, Novartis, Pfizer, DFG, EU, BMBF, and Insmed; he is on the advisory board for AstraZeneca, Boehringer, Bayer, Gilead, GSK, Insmed, Novartis, Pfizer, Roche; he is a member of the European Respiratory Society, German Society of Pneumology, and Paul Ehrlich Gesellschaft. Dr. Zimmerman is a member of the ACP, AACP, and WFPICCS. Dr. Levy is a legal consultant for a few cases involving sepsis and serves as co-chair of the Surviving Sepsis Campaign Steering Committee. The remaining authors have disclosed that they do not have any potential conflicts of interest.

\section{Publisher's Note}

Springer Nature remains neutral with regard to jurisdictional claims in published maps and institutional affiliations.

\section{Received: 6 June 2021 Accepted: 5 August 2021}

\section{Published online: 2 October 2021}

\section{References}

1. Singer M, Deutschman CS, Seymour CW et al (2016) The Third International Consensus definitions for sepsis and septic shock (Sepsis-3). JAMA 315(8):801-810

2. Fleischmann C, Scherag A, Adhikari NK et al (2016) Assessment of global incidence and mortality of hospital-treated sepsis. Current estimates and limitations. Am J Respir Crit Care Med 193(3):259-272

3. Fleischmann-Struzek C, Mellhammar L, Rose N et al (2020) Incidence and mortality of hospital- and ICU-treated sepsis: results from an updated and expanded systematic review and meta-analysis. Intensive Care Med 46(8):1552-1562

4. Rhee C, Dantes R, Epstein L et al (2017) Incidence and trends of sepsis in US hospitals using clinical vs claims data, 2009-2014. JAMA 318(13):1241-1249

5. Seymour CW, Liu VX, Iwashyna TJ et al (2016) Assessment of clinical criteria for sepsis: for the third international consensus definitions for sepsis and septic shock (Sepsis-3). JAMA 315(8):762-774

6. Levy MM, Fink MP, Marshall JC et al (2003) 2001 SCCM/ESICM/ACCP/ ATS/SIS International Sepsis Definitions Conference. Crit Care Med 31(4):1250-1256
7. Dellinger RP, Levy MM, Rhodes A et al (2013) Surviving Sepsis Campaign: international guidelines for management of severe sepsis and septic shock, 2012. Intensive Care Med 39(2):165-228

8. Dellinger RP, Levy MM, Rhodes A et al (2013) Surviving sepsis campaign: international guidelines for management of severe sepsis and septic shock: 2012. Crit Care Med 41(2):580-637

9. Dellinger RP, Carlet JM, Masur H et al (2004) Surviving Sepsis Campaign guidelines for management of severe sepsis and septic shock. Crit Care Med 32(3):858-873

10. Dellinger RP, Levy MM, Carlet JM (2008) Surviving Sepsis Campaign: International guidelines for management of severe sepsis and septic shock: 2008 (vol 36, pg 296, 2008). Crit Care Med 36(4):1394-1396

11. Dellinger RP, Levy MM, Carlet JM et al (2008) surviving sepsis campaign: International guidelines for management of severe sepsis and septic shock: 2008. Crit Care Med 36(1):296-327

12. Rhodes A, Evans LE, Alhazzani W et al (2017) Surviving sepsis campaign: international guidelines for management of sepsis and septic shock: 2016. Crit Care Med 45(3):486-552

13. Rhodes A, Evans LE, Alhazzani W et al (2017) surviving sepsis campaign: international guidelines for management of sepsis and septic shock: 2016. Intensive Care Med 43(3):304-377

14. Weiss SL, Peters MJ, Alhazzani W et al (2020) Surviving sepsis campaign international guidelines for the management of septic shock and sepsis-associated organ dysfunction in children. Intensive Care Med 46(Suppl 1):10-67

15. Weiss SL, Peters MJ, Alhazzani W et al (2020) Surviving sepsis campaign international guidelines for the management of septic shock and sepsis-associated organ dysfunction in children. Pediatr Crit Care Med 21(2):e52-e106

16. Guyatt GH, Oxman AD, Kunz R et al (2011) GRADE guidelines: 2 . Framing the question and deciding on important outcomes. J Clin Epidemiol 64(4):395-400

17. Akl EA, Johnston BC, Alonso-Coello P et al (2013) Addressing dichotomous data for participants excluded from trial analysis: a guide for systematic reviewers. PLoS One 8(2):e57132

18. DerSimonian R, Laird N (1986) Meta-analysis in clinical trials. Control Clin Trials 7(3):177-188

19. Guyatt GH, Oxman AD, Vist GE et al (2008) GRADE: an emerging consensus on rating quality of evidence and strength of recommendations. BMJ 336(7650):924-926

20. Balshem H, Helfand M, Schunemann HJ et al (2011) GRADE guidelines: 3. Rating the quality of evidence. J Clin Epidemiol 64(4):401-406

21. Andrews J, Guyatt G, Oxman AD et al (2013) GRADE guidelines: 14. Going from evidence to recommendations: the significance and presentation of recommendations. J Clin Epidemiol 66(7):719-725

22. Guyatt GH, Oxman AD, Santesso N et al (2013) GRADE guidelines: 12. Preparing summary of findings tables-binary outcomes. J Clin Epidemiol 66(2):158-172

23. Schunemann HJ, Wiercioch W, Brozek J et al (2017) GRADE Evidence to Decision (EtD) frameworks for adoption, adaptation, and de novo development of trustworthy recommendations: GRADE-ADOLOPMENT. J Clin Epidemiol 81:101-110

24. Guyatt GH, Schunemann HJ, Djulbegovic B et al (2015) Guideline panels should not GRADE good practice statements. J Clin Epidemiol 68(5):597-600

25. Dellinger RP (2015) The future of sepsis performance improvement. Crit Care Med 43(9):1787-1789

26. Schorr C, Odden A, Evans L et al (2016) Implementation of a multicenter performance improvement program for early detection and treatment of severe sepsis in general medical-surgical wards. J Hosp Med 11(S1):S32-S39

27. Damiani E, Donati A, Serafini $G$ et al (2015) Effect of performance improvement programs on compliance with sepsis bundles and mortality: a systematic review and meta-analysis of observational studies. PLoS One 10(5):e0125827

28. Alberto L, Marshall AP, Walker R et al (2017) Screening for sepsis in general hospitalized patients: a systematic review. J Hosp Infect 96(4):305-315

29. Bhattacharjee P, Edelson DP, Churpek MM (2017) Identifying patients with sepsis on the hospital wards. Chest 151(4):898-907 
30. Makam AN, Nguyen OK, Auerbach AD (2015) Diagnostic accuracy and effectiveness of automated electronic sepsis alert systems: a systematic review. J Hosp Med 10(6):396-402

31. Warttig S, Alderson P, Evans DJ et al (2018) Automated monitoring compared to standard care for the early detection of sepsis in critically ill patients. Cochrane Database oSyste Rev. 6(6):CD012404-CD

32. Islam MM, Nasrin T, Walther BA et al (2019) Prediction of sepsis patients using machine learning approach: a meta-analysis. Comput Methods Programs Biomed 170:1-9

33. Downing NL, Rolnick J, Poole SF et al (2019) Electronic health recordbased clinical decision support alert for severe sepsis: a randomised evaluation. BMJ Qual Saf 28(9):762-768

34. Hooper MH, Weavind L, Wheeler AP et al (2012) Randomized trial of automated, electronic monitoring to facilitate early detection of sepsis in the intensive care unit*. Crit Care Med 40(7):2096-2101

35. Shimabukuro DW, Barton CW, Feldman MD et al (2017) Effect of a machine learning-based severe sepsis prediction algorithm on patient survival and hospital length of stay: a randomised clinical trial. BMJ Open Respir Res 4(1):e000234

36. Rao TSS, Radhakrishnan R, Andrade C (2011) Standard operating procedures for clinical practice. Indian J Psychiatry 53(1):1-3

37. Osborn TM (2017) Severe sepsis and septic shock trials (ProCESS, ARISE, ProMISe): what is optimal resuscitation? Crit Care Clin 33(2):323-344

38. Kahn JM, Davis BS, Yabes JG et al (2019) Association between statemandated protocolized sepsis care and in-hospital mortality among adults with sepsis. JAMA 322(3):240-250

39. Morton B, Stolbrink M, Kagima W et al (2018) The early recognition and management of sepsis in Sub-Saharan African adults: a systematic review and meta-analysis. Int J Environ Res Public Health 15(9):2017

40. Fernando SM, Tran A, Taljaard M et al (2018) Prognostic accuracy of the quick sequential organ failure assessment for mortality in patients with suspected infection: a systematic review and meta-analysis. Ann Intern Med 168(4):266-275

41. Herwanto V, Shetty A, Nalos M et al (2019) Accuracy of quick sequential organ failure assessment score to predict sepsis mortality in 121 studies including 1,716,017 individuals: a systematic review and meta-analysis. Crit Care Explor. 1(9):e0043

42. Serafim R, Gomes JA, Salluh J et al (2018) A comparison of the QuickSOFA and systemic inflammatory response syndrome criteria for the diagnosis of sepsis and prediction of mortality: a systematic review and meta-analysis. Chest 153(3):646-655

43. Cinel I, Kasapoglu US, Gul F et al (2020) The initial resuscitation of septic shock. J Crit Care 57:108-117

44. Liu VX, Lu Y, Carey KA et al (2020) Comparison of early warning scoring systems for hospitalized patients with and without infection at risk for in-hospital mortality and transfer to the intensive care unit. JAMA Netw Open 3(5):e205191

45. Borthwick HA, Brunt LK, Mitchem KL et al (2012) Does lactate measurement performed on admission predict clinical outcome on the intensive care unit? A concise systematic review. Ann Clin Biochem 49(Pt 4):391-394

46. Liu G, An Y, Yi X et al (2017) Early lactate levels for prediction of mortality in patients with sepsis or septic shock: a meta-analysis. Int J Exp Med 10:37-47

47. Levy MM, Evans LE, Rhodes A (2018) The surviving sepsis campaign bundle: 2018 update. Crit Care Med 46(6):997-1000

48. Levy MM, Evans LE, Rhodes A (2018) The surviving sepsis campaign bundle: 2018 update. Intensive Care Med 44(6):925-928

49. Shankar-Hari M, Phillips GS, Levy ML et al (2016) Developing a new definition and assessing new clinical criteria for septic shock: for the third international consensus definitions for sepsis and septic shock (Sepsis-3). JAMA 315(8):775-787

50. Contenti J, Corraze H, Lemoel F et al (2015) Effectiveness of arterial, venous, and capillary blood lactate as a sepsis triage tool in ED patients. Am J Emerg Med 33(2):167-172

51. Karon BS, Tolan NV, Wockenfus AM et al (2017) Evaluation of lactate, white blood cell count, neutrophil count, procalcitonin and immature granulocyte count as biomarkers for sepsis in emergency department patients. Clin Biochem 50(16-17):956-958

52. Ljungstrom L, Pernestig AK, Jacobsson G et al (2017) Diagnostic accuracy of procalcitonin, neutrophil-lymphocyte count ratio, C-reactive protein, and lactate in patients with suspected bacterial sepsis. PLoS One 12(7):e0181704

53. Morris E, McCartney D, Lasserson D et al (2017) Point-of-care lactate testing for sepsis at presentation to health care: a systematic review of patient outcomes. Br J Gen Pract 67(665):e859-e870

54. Abdu M, Wilson A, Mhango C et al (2018) Resource availability for the management of maternal sepsis in Malawi, other low-income countries, and lower-middle-income countries. Int J Gynaecol Obstet 140(2):175-183

55. Baelani I, Jochberger S, Laimer T et al (2011) Availability of critical care resources to treat patients with severe sepsis or septic shock in Africa: a self-reported, continent-wide survey of anaesthesia providers. Crit Care 15(1):R10

56. Baelani I, Jochberger S, Laimer T et al (2012) Identifying resource needs for sepsis care and guideline implementation in the Democratic Republic of the Congo: a cluster survey of 66 hospitals in four eastern provinces. Middle East J Anaesthesiol 21(4):559-575

57. Bataar O, Lundeg G, Tsenddorj G et al (2010) Nationwide survey on resource availability for implementing current sepsis guidelines in Mongolia. Bull World Health Organ 88(11):839-846

58. Hernandez G, Ospina-Tascon GA, Damiani LP et al (2019) Effect of a resuscitation strategy targeting peripheral perfusion status vs serum lactate levels on 28-day mortality among patients with septic shock: the ANDROMEDA-SHOCK Randomized Clinical Trial. JAMA 321(7):654-664

59. Machado FR, Cavalcanti AB, Bozza FA et al (2017) The epidemiology of sepsis in Brazilian intensive care units (the Sepsis PREvalence Assessment Database, SPREAD): an observational study. Lancet Infect Dis 17(11):1180-1189

60. Shrestha GS, Kwizera A, Lundeg G et al (2017) International Surviving Sepsis Campaign quidelines 2016: the perspective from low-income and middle-income countries. Lancet Infect Dis 17(9):893-895

61. Taniguchi LU, Azevedo LCP, Bozza FA et al (2019) Availability of resources to treat sepsis in Brazil: a random sample of Brazilian institutions. Rev Bras Ter Intensiva 31(2):193-201

62. Levy MM, Dellinger RP, Townsend SR et al (2010) The Surviving Sepsis Campaign: results of an international guideline-based performance improvement program targeting severe sepsis. Intensive Care Med 36(2):222-231

63. Kuttab HI, Lykins JD, Hughes MD et al (2019) Evaluation and predictors of fluid resuscitation in patients with severe sepsis and septic shock. Crit Care Med 47(11):1582-1590

64. Investigators P, Yealy DM, Kellum JA et al (2014) A randomized trial of protocol-based care for early septic shock. N Engl J Med 370(18):1683-1693

65. Peake SL, Delaney A, Bellomo R et al (2015) Goal-directed resuscitation in septic shock. N Engl J Med 372(2):190-191

66. Mouncey PR, Osborn TM, Power GS et al (2015) Trial of early, goaldirected resuscitation for septic shock. N Engl J Med 372(14):1301-1311

67. Rowan KM, Angus DC, Bailey M et al (2017) Early, goal-directed therapy for septic shock - a patient-level meta-analysis. N Engl J Med 376(23):2223-2234

68. Ehrman RR, Gallien JZ, Smith RK et al (2019) Resuscitation quided by volume responsiveness does not reduce mortality in sepsis: a metaanalysis. Crit Care Explor. 1(5):e0015

69. Andrews B, Semler MW, Muchemwa L et al (2017) Effect of an early resuscitation protocol on in-hospital mortality among adults with sepsis and hypotension: a randomized clinical trial. JAMA 318(13):1233-1240

70. Aya HD, Rhodes A, Chis Ster I et al (2017) Hemodynamic effect of different doses of fluids for a fluid challenge: a quasi-randomized controlled study. Crit Care Med 45(2):e161-e168

71. Cherpanath TG, Hirsch A, Geerts BF et al (2016) Predicting fluid responsiveness by passive leg raising: a systematic review and meta-analysis of 23 clinical trials. Crit Care Med 44(5):981-991

72. Misango D, Pattnaik R, Baker T et al (2017) Haemodynamic assessment and support in sepsis and septic shock in resource-limited settings. Trans R Soc Trop Med Hyg 111(11):483-489

73. Levy B (2006) Lactate and shock state: the metabolic view. Curr Opin Crit Care 12(4):315-321 
74. Gu WJ, Zhang Z, Bakker J (2015) Early lactate clearance-guided therapy in patients with sepsis: a meta-analysis with trial sequential analysis of randomized controlled trials. Intensive Care Med 41(10):1862-1863

75. Simpson SQ, Gaines M, Hussein Y et al (2016) Early goal-directed therapy for severe sepsis and septic shock: a living systematic review. J Crit Care 36:43-48

76. Cecconi M, Hernandez G, Dunser M et al (2019) Fluid administration for acute circulatory dysfunction using basic monitoring: narrative review and expert panel recommendations from an ESICM task force. Intensive Care Med 45(1):21-32

77. Lara B, Enberg L, Ortega M et al (2017) Capillary refill time during fluid resuscitation in patients with sepsis-related hyperlactatemia at the emergency department is related to mortality. PLoS One 12(11):e0188548

78. Shrestha GS, Dunser M, Mer M (2017) The forgotten value of the clinical examination to individualize and guide fluid resuscitation in patients with sepsis. Crit Care 21(1):306

79. LeDoux D, Astiz ME, Carpati CM et al (2000) Effects of perfusion pres sure on tissue perfusion in septic shock. Crit Care Med 28(8):2729-2732

80. Asfar P, Meziani F, Hamel JF et al (2014) High versus low blood-pressure target in patients with septic shock. N Engl J Med 370(17):1583-1593

81. Hylands M, Moller MH, Asfar P et al (2017) A systematic review of vasopressor blood pressure targets in critically ill adults with hypotension. Can J Anaesth 64(7):703-715

82. Lamontagne F, Meade MO, Hebert PC et al (2016) Higher versus lower blood pressure targets for vasopressor therapy in shock: a multicentre pilot randomized controlled trial. Intensive Care Med 42(4):542-550

83. Lamontagne F, Richards-Belle A, Thomas K et al (2020) Effect of reduced exposure to vasopressors on 90-day mortality in older critically ill patients with vasodilatory hypotension: a randomized clinical trial. JAMA 323(10):938-949

84. Mohr NM, Wessman BT, Bassin B et al (2020) Boarding of critically ill patients in the emergency department. Crit Care Med 48(8):1180-1187

85. Cardoso LT, Grion CM, Matsuo T et al (2011) Impact of delayed admission to intensive care units on mortality of critically ill patients: a cohort study. Crit Care 15(1):R28

86. Groenland CNL, Termorshuizen F, Rietdijk WJR et al (2019) emergency department to icu time is associated with hospital mortality: a registry analysis of 14,788 patients from six University Hospitals in The Netherlands. Crit Care Med 47(11):1564-1571

87. Chalfin DB, Trzeciak S, Likourezos A et al (2007) Impact of delayed transfer of critically ill patients from the emergency department to the intensive care unit. Crit Care Med 35(6):1477-1483

88. Harris S, Singer M, Sanderson C et al (2018) Impact on mortality of prompt admission to critical care for deteriorating ward patients: an instrumental variable analysis using critical care bed strain. Intensive Care Med 44(5):606-615

89. Montgomery A, Panagopoulou E, Kehoe I et al (2011) Connecting organisational culture and quality of care in the hospital: is job burnout the missing link? J Health Organ Manag 25(1):108-123

90. Klein Klouwenberg PM, Cremer OL, van Vught LA et al (2015) Likelihood of infection in patients with presumed sepsis at the time of intensive care unit admission: a cohort study. Crit Care 19:319

91. Levin PD, Idrees S, Sprung CL et al (2012) Antimicrobial use in the ICU: indications and accuracy —an observational trial. J Hosp Med 7(9):672-678

92. Minderhoud TC, Spruyt C, Huisman S et al (2017) Microbiological outcomes and antibiotic overuse in Emergency Department patients with suspected sepsis. Neth J Med 75(5):196-203

93. Heffner AC, Horton JM, Marchick MR et al (2010) Etiology of illness in patients with severe sepsis admitted to the hospital from the emergency department. Clin Infect Dis 50(6):814-820

94. Tidswell R, Parker T, Brealey D, et al (2020) Sepsis-the broken code how accurately is sepsis being diagnosed? J Infect 81(6):e31-e32.

95. Deuster S, Roten I, Muehlebach S (2010) Implementation of treatment guidelines to support judicious use of antibiotic therapy. J Clin Pharm Ther 35(1):71-78

96. Ferrer R, Artigas A, Suarez D et al (2009) Effectiveness of treatments for severe sepsis: a prospective, multicenter, observational study. Am 」 Respir Crit Care Med 180(9):861-866
97. Kalil AC, Johnson DW, Lisco SJ et al (2017) Early goal-directed therapy for sepsis: a novel solution for discordant survival outcomes in clinical trials. Crit Care Med 45(4):607-614

98. Seymour CW, Gesten F, Prescott HC et al (2017) Time to treatment and mortality during mandated emergency care for sepsis. N Engl J Med 376(23):2235-2244

99. Klompas M, Calandra T, Singer M (2018) Antibiotics for sepsis-finding the equilibrium. JAMA 320(14):1433-1434

100. Prescott HC, Iwashyna TJ (2019) Improving sepsis treatment by embracing diagnostic uncertainty. Ann Am Thorac Soc 16(4):426-429

101. Baggs J, Jernigan JA, Halpin AL et al (2018) Risk of subsequent sepsis within 90 days after a hospital stay by type of antibiotic exposure. Clin Infect Dis 66(7):1004-1012

102. Branch-Elliman W, O'Brien W, Strymish J et al (2019) Association of duration and type of surgical prophylaxis with antimicrobial-associated adverse events. JAMA Surg 154(7):590-598

103. Hranjec T, Rosenberger LH, Swenson B et al (2012) Aggressive versus conservative initiation of antimicrobial treatment in critically ill surgical patients with suspected intensive-care-unit-acquired infection: a quasi-experimental, before and after observational cohort study. Lancet Infect Dis 12(10):774-780

104. Ong DSY, Frencken JF, Klein Klouwenberg PMC et al (2017) Short-course adjunctive gentamicin as empirical therapy in patients with severe sepsis and septic shock: a prospective observational cohort study. Clin Infect Dis 64(12):1731-1736

105. Tamma PD, Avdic E, Li DX et al (2017) Association of adverse events with antibiotic use in hospitalized patients. JAMA Intern Med 177(9):1308-1315

106. Teshome BF, Vouri SM, Hampton N et al (2019) Duration of exposure to antipseudomonal beta-lactam antibiotics in the critically ill and development of new resistance. Pharmacotherapy 39(3):261-270

107. Contou D, Roux D, Jochmans S et al (2016) Septic shock with no diagnosis at 24 hours: a pragmatic multicenter prospective cohort study. Crit Care 20(1):360

108. Rhee C, Kadri SS, Danner RL et al (2016) Diagnosing sepsis is subjective and highly variable: a survey of intensivists using case vignettes. Crit Care 20:89

109. Kumar A, Roberts D, Wood KE et al (2006) Duration of hypotension before initiation of effective antimicrobial therapy is the critical determinant of survival in human septic shock. Crit Care Med 34(6):1589-1596

110. Liu VX, Fielding-Singh V, Greene JD et al (2017) The timing of early antibiotics and hospital mortality in sepsis. Am J Respir Crit Care Med 196(7):856-863

111. Peltan ID, Brown SM, Bledsoe JR et al (2019) ED door-to-antibiotic time and long-term mortality in sepsis. Chest 155(5):938-946

112. Abe T, Kushimoto S, Tokuda Y et al (2019) Implementation of earlier antibiotic administration in patients with severe sepsis and septic shock in Japan: a descriptive analysis of a prospective observational study. Crit Care 23(1):360

113. Gaieski DF, Mikkelsen ME, Band RA et al (2010) Impact of time to antibiotics on survival in patients with severe sepsis or septic shock in whom early goal-directed therapy was initiated in the emergency department. Crit Care Med 38(4):1045-1053

114. Ko BS, Choi SH, Kang GH et al (2020) Time to antibiotics and the outcome of patients with septic shock: a propensity score analysis. Am J Med 133(4):485-91e4

115. Puskarich MA, Trzeciak S, Shapiro NI et al (2011) Association between timing of antibiotic administration and mortality from septic shock in patients treated with a quantitative resuscitation protocol. Crit Care Med 39(9):2066-2071

116. Rothrock SG, Cassidy DD, Barneck M, et al (2020) Outcome of immediate versus early antibiotics in severe sepsis and septic shock: a systematic review and meta-analysis. Ann Emerg Med 76(4):427-441

117. Ryoo SM, Kim WY, Sohn CH et al (2015) Prognostic value of timing of antibiotic administration in patients with septic shock treated with early quantitative resuscitation. Am J Med Sci 349(4):328-333

118. Weinberger J, Rhee C, Klompas M (2020) A critical analysis of the literature on time-to-antibiotics in suspected sepsis. J Infect Dis 222(Supplement_2):S110-S118 
119. Alam N, Oskam E, Stassen PM et al (2018) Prehospital antibiotics in the ambulance for sepsis: a multicentre, open label, randomised trial. Lancet Respir Med 6(1):40-50

120. Bloos F, Ruddel H, Thomas-Ruddel D et al (2017) Effect of a multifaceted educational intervention for anti-infectious measures on sepsis mortality: a cluster randomized trial. Intensive Care Med 43(11):1602-1612

121. Chalya PL, Mabula JB, Koy M et al (2012) Typhoid intestinal perforations at a University teaching hospital in Northwestern Tanzania: a surgical experience of 104 cases in a resource-limited setting. World J Emerg Surg 7:4

122. Phua J, Koh Y, Du B et al (2011) Management of severe sepsis in patients admitted to Asian intensive care units: prospective cohort study. BMJ 342: $\mathrm{d} 3245$

123. Thwaites CL, Lundeg G, Dondorp AM et al (2016) Recommendations for infection management in patients with sepsis and septic shock in resource-limited settings. Intensive Care Med 42(12):2040-2042

124. Urayeneza O, Mujyarugamba P, Rukemba Z et al (2018) Increasing evidence-based interventions in patients with acute infections in a resource-limited setting: a before-and-after feasibility trial in Gitwe, Rwanda. Crit Care Med 46(8):1357-1366

125. Urayeneza O, Mujyarugamba P, Rukemba Z et al (2018) Increasing evidence-based interventions in patients with acute infections in a resource-limited setting: a before-and-after feasibility trial in Gitwe, Rwanda. Intensive Care Med 44(9):1436-1446

126. Yokota PK, Marra AR, Martino MD et al (2014) Impact of appropriate antimicrobial therapy for patients with severe sepsis and septic shocka quality improvement study. PLoS One 9(11):e104475

127. Peng F, Chang W, Xie JF et al (2019) Ineffectiveness of procalcitoninguided antibiotic therapy in severely critically ill patients: a meta-analysis. Int J Infect Dis 85:158-166

128. Wacker C, Prkno A, Brunkhorst FM et al (2013) Procalcitonin as a diagnostic marker for sepsis: a systematic review and meta-analysis. Lancet Infect Dis 13(5):426-435

129. Jensen JU, Hein L, Lundgren B et al (2011) Procalcitonin-guided interventions against infections to increase early appropriate antibiotics and improve survival in the intensive care unit: a randomized trial. Crit Care Med 39(9):2048-2058

130. Layios N, Lambermont B, Canivet JL et al (2012) Procalcitonin usefulness for the initiation of antibiotic treatment in intensive care unit patients. Crit Care Med 40(8):2304-2309

131. Najafi A, Khodadadian A, Sanatkar M et al (2015) The comparison of procalcitonin guidance administer antibiotics with empiric antibiotic therapy in critically ill patients admitted in intensive care unit. Acta Med Iran 53(9):562-567

132. Metlay JP, Waterer GW, Long AC et al (2019) Diagnosis and treatment of adults with community-acquired pneumonia. An Official Clinical Practice Guideline of the American Thoracic Society and Infectious Diseases Society of America. Am J Respir Crit Care Med 200(7):e45-e67

133. Vincent JL, Sakr Y, Singer M, et al (2020) Prevalence and outcomes of infection among patients in intensive care units in 2017. JAMA 323(15):1478-1487

134. Jernigan JA, Hatfield KM, Wolford $\mathrm{H}$ et al (2020) Multidrug-resistant bacterial infections in U.S. hospitalized patients, 2012-2017. N Engl J Med 382(14):1309-1319

135. Jones M, Jernigan JA, Evans ME et al (2019) Vital Signs: trends in Staphylococcus aureus infections in veterans affairs medical centers-United States, 2005-2017. MMWR Morb Mortal Wkly Rep 68(9):220-224

136. Aliberti S, Reyes LF, Faverio P et al (2016) Global initiative for meticillinresistant Staphylococcus aureus pneumonia (GLIMP): an international, observational cohort study. Lancet Infect Dis 16(12):1364-1376

137. Rhee C, Kadri SS, Dekker JP et al (2020) Prevalence of antibiotic-resistant pathogens in culture-proven sepsis and outcomes associated with inadequate and broad-spectrum empiric antibiotic use. JAMA Netw Open 3(4):e202899

138. Callejo-Torre F, Eiros Bouza JM, Olaechea Astigarraga P et al (2016) Risk factors for methicillin-resistant Staphylococcus aureus colonisation or infection in intensive care units and their reliability for predicting MRSA on ICU admission. Infez Med 24(3):201-209
139. Epstein L Mu Y, Belflower R et al (2016) Risk factors for invasive methicillin-resistant Staphylococcus aureus infection after recent discharge from an acute-care hospitalization, 2011-2013. Clin Infect Dis 62(1):45-52

140. Shorr AF, Myers DE, Huang DB et al (2013) A risk score for identifying methicillin-resistant Staphylococcus aureus in patients presenting to the hospital with pneumonia. BMC Infect Dis 13:268

141. Torre-Cisneros J, Natera C, Mesa F et al (2018) Clinical predictors of methicillin-resistant Staphylococcus aureus in nosocomial and healthcare-associated pneumonia: a multicenter, matched case-control study. Eur J Clin Microbiol Infect Dis 37(1):51-56

142. Wooten DA, Winston LG (2013) Risk factors for methicillin-resistant Staphylococcus aureus in patients with community-onset and hospitalonset pneumonia. Respir Med 107(8):1266-1270

143. Gasch O, Camoez M, Dominguez MA et al (2013) Predictive factors for early mortality among patients with methicillin-resistant Staphylococcus aureus bacteraemia. J Antimicrob Chemother 68(6):1423-1430

144. Gasch O, Camoez M, Dominguez MA et al (2013) Predictive factors for mortality in patients with methicillin-resistant Staphylococcus aureus bloodstream infection: impact on outcome of host, microorganism and therapy. Clin Microbiol Infect 19(11):1049-1057

145. Lodise TP, McKinnon PS, Swiderski L et al (2003) Outcomes analysis of delayed antibiotic treatment for hospital-acquired Staphylococcus aureus bacteremia. Clin Infect Dis 36(11):1418-1423

146. Paul M, Kariv G, Goldberg E et al (2010) Importance of appropriate empirical antibiotic therapy for methicillin-resistant Staphylococcus aureus bacteraemia. J Antimicrob Chemother 65(12):2658-2665

147. Schramm GE, Johnson JA, Doherty JA et al (2006) Methicillin-resistant Staphylococcus aureus sterile-site infection: the importance of appropriate initial antimicrobial treatment. Crit Care Med 34(8):2069-2074

148. Fang CT, Shau WY, Hsueh PR et al (2006) Early empirical glycopeptide therapy for patients with methicillin-resistant Staphylococcus aureus bacteraemia: impact on the outcome. J Antimicrob Chemother 57(3):511-519

149. Gomez J, Garcia-Vazquez E, Banos R et al (2007) Predictors of mortality in patients with methicillin-resistant Staphylococcus aureus (MRSA) bacteraemia: the role of empiric antibiotic therapy. Eur J Clin Microbiol Infect Dis 26(4):239-245

150. Griffin AT, Peyrani P, Wiemken TL et al (2013) Empiric therapy directed against MRSA in patients admitted to the intensive care unit does not improve outcomes in community-acquired pneumonia. Infection 41(2):517-523

151. Kett DH, Cano E, Quartin AA et al (2011) Implementation of guidelines for management of possible multidrug-resistant pneumonia in intensive care: an observational, multicentre cohort study. Lancet Infect Dis 11(3):181-189

152. Khatib R, Saeed S, Sharma M et al (2006) Impact of initial antibiotic choice and delayed appropriate treatment on the outcome of Staphylococcus aureus bacteremia. Eur J Clin Microbiol Infect Dis 25(3):181-185

153. Kim SH, Park WB, Lee KD et al (2004) Outcome of inappropriate initial antimicrobial treatment in patients with methicillin-resistant Staphylococcus aureus bacteraemia. J Antimicrob Chemother 54(2):489-497

154. Yoon YK, Park DW, Sohn JW et al (2016) Effects of inappropriate empirical antibiotic therapy on mortality in patients with healthcareassociated methicillin-resistant Staphylococcus aureus bacteremia: a propensity-matched analysis. BMC Infect Dis 16:331

155. Jones BE, Ying J, Stevens V, et al (2020) Empirical anti-MRSA vs standard antibiotic therapy and risk of 30-day mortality in patients hospitalized for pneumonia. JAMA Intern Med 180(4):552-560

156. Webb BJ, Sorensen J, Jephson A, et al (2019) Broad-spectrum antibiotic use and poor outcomes in community-onset pneumonia: a cohort study. Eur Respir J 54(1):1900057

157. Baby N, Faust AC, Smith T, et al (2017) Nasal methicillin-resistant Staphylococcus aureus (MRSA) PCR testing reduces the duration of MRSA-targeted therapy in patients with suspected MRSA pneumonia. Antimicrob Agents Chemother 61(4):e02432-16

158. Cowley MC, Ritchie DJ, Hampton N et al (2019) Outcomes associated with de-escalating therapy for methicillin-resistant Staphylococcus aureus in culture-negative nosocomial pneumonia. Chest 155(1):53-59 
159. Paonessa JR, Shah RD, Pickens Cl et al (2019) Rapid detection of methicillin-resistant Staphylococcus aureus in BAL: a pilot randomized controlled trial. Chest 155(5):999-1007

160. Sjovall F, Perner A, Hylander MM (2017) Empirical mono- versus combination antibiotic therapy in adult intensive care patients with severe sepsis - a systematic review with meta-analysis and trial sequential analysis. J Infect 74(4):331-344

161. Brunkhorst FM, Oppert M, Marx G et al (2012) Effect of empirical treatment with moxifloxacin and meropenem vs meropenem on sepsisrelated organ dysfunction in patients with severe sepsis: a randomized trial. JAMA 307(22):2390-2399

162. Alevizakos M, Karanika S, Detsis M et al (2016) Colonisation with extended-spectrum beta-lactamase-producing Enterobacteriaceae and risk for infection among patients with solid or haematological malignancy: a systematic review and meta-analysis. Int J Antimicrob Agents 48(6):647-654

163. Rottier WC, Bamberg YR, Dorigo-Zetsma JW et al (2015) Predictive value of prior colonization and antibiotic use for third-generation cephalosporin-resistant enterobacteriaceae bacteremia in patients with sepsis. Clin Infect Dis 60(11):1622-1630

164. Rottier WC, van Werkhoven CH, Bamberg YRP et al (2018) Development of diagnostic prediction tools for bacteraemia caused by third-generation cephalosporin-resistant enterobacteria in suspected bacterial infections: a nested case-control study. Clin Microbiol Infect 24(12):1315-1321

165. Arulkumaran N, Routledge M, Schlebusch S et al (2020) Antimicrobialassociated harm in critical care: a narrative review. Intensive Care Med 46(2):225-235

166. Bassetti M, Righi E, Ansaldi F et al (2014) A multicenter study of septic shock due to candidemia: outcomes and predictors of mortality. Intensive Care Med 40(6):839-845

167. Kollef M, Micek S, Hampton N et al (2012) Septic shock attributed to Candida infection: importance of empiric therapy and source control. Clin Infect Dis 54(12):1739-1746

168. Magill SS, Edwards JR, Bamberg W et al (2014) Multistate pointprevalence survey of health care-associated infections. N Engl J Med 370(13):1198-1208

169. Mean M, Marchetti $O$, Calandra T (2008) Bench-to-bedside review: Candida infections in the intensive care unit. Crit Care 12(1):204

170. Pappas PG, Kauffman CA, Andes DR et al (2016) Clinical practice guideline for the management of candidiasis: 2016 Update by the Infectious Diseases Society of America. Clin Infect Dis 62(4):e1-50

171. Garey KW, Rege M, Pai MP et al (2006) Time to initiation of fluconazole therapy impacts mortality in patients with candidemia: a multi-institutional study. Clin Infect Dis 43(1):25-31

172. Marriott DJ, Playford EG, Chen S et al (2009) Determinants of mortality in non-neutropenic ICU patients with candidaemia. Crit Care 13(4):R115

173. Morrell M, Fraser VJ, Kollef MH (2005) Delaying the empiric treatment of candida bloodstream infection until positive blood culture results are obtained: a potential risk factor for hospital mortality. Antimicrob Agents Chemother 49(9):3640-3645

174. Timsit JF, Azoulay E, Schwebel C et al (2016) Empirical micafungin treatment and survival without invasive fungal infection in adults with ICU-acquired sepsis, candida colonization, and multiple organ failure: the EMPIRICUS Randomized Clinical Trial. JAMA 316(15):1555-1564

175. Freifeld AG, Bow EJ, Sepkowitz KA et al (2011) Clinical practice guideline for the use of antimicrobial agents in neutropenic patients with cancer: 2010 update by the infectious diseases society of america. Clin Infect Dis 52(4):e56-93

176. Taplitz RA, Kennedy EB, Bow EJ et al (2018) Outpatient management of fever and neutropenia in adults treated for malignancy: American Society of Clinical Oncology and Infectious Diseases Society of America Clinical Practice Guideline Update. J Clin Oncol 36(14):1443-1453

177. Clancy CJ, Nguyen MH (2018) Diagnosing invasive candidiasis. J Clin Microbiol 56(5):e01909-17

178. Kullberg BJ, Arendrup MC (2015) Invasive candidiasis. N Engl J Med 373(15):1445-1456

179. Sandven P, Qvist H, Skovlund E et al (2002) Significance of Candida recovered from intraoperative specimens in patients with intra-abdominal perforations. Crit Care Med 30(3):541-547
180. Hachem R, Hanna H, Kontoyiannis D et al (2008) The changing epidemiology of invasive candidiasis: Candida glabrata and Candida krusei as the leading causes of candidemia in hematologic malignancy. Cancer 112(11):2493-2499

181. Horn DL, Neofytos D, Anaissie EJ et al (2009) Epidemiology and outcomes of candidemia in 2019 patients: data from the prospective antifungal therapy alliance registry. Clin Infect Dis 48(12):1695-1703

182. Andes DR, Safdar N, Baddley JW et al (2012) Impact of treatment strategy on outcomes in patients with candidemia and other forms of invasive candidiasis: a patient-level quantitative review of randomized trials. Clin Infect Dis 54(8):1110-1122

183. Kett DH, Azoulay E, Echeverria PM et al (2011) Candida bloodstream infections in intensive care units: analysis of the extended prevalence of infection in intensive care unit study. Crit Care Med 39(4):665-670

184. Cleveland AA, Harrison LH, Farley MM et al (2015) Declining incidence of candidemia and the shifting epidemiology of Candida resistance in two US metropolitan areas, 2008-2013: results from population-based surveillance. PLoS One 10(3):e0120452

185. Zhang AY, Shrum S, Williams S et al (2020) The changing epidemiology of candidemia in the united states: injection drug use as an increasingly common risk factor-active surveillance in selected sites, United States, 2014-2017. Clin Infect Dis 71(7):1732-1737

186. Blumberg HM, Jarvis WR, Soucie JM et al (2001) Risk factors for candidal bloodstream infections in surgical intensive care unit patients: the NEMIS prospective multicenter study. The National Epidemiology of Mycosis Survey. Clin Infect Dis 33(2):177-186

187. Fan D, Coughlin LA, Neubauer MM et al (2015) Activation of HIF-1alpha and LL-37 by commensal bacteria inhibits Candida albicans colonization. Nat Med 21(7):808-814

188. Chow JK, Golan Y, Ruthazer R et al (2008) Risk factors for albicans and non-albicans candidemia in the intensive care unit. Crit Care Med 36(7):1993-1998

189. Ostrosky-Zeichner L, Pappas PG (2006) Invasive candidiasis in the intensive care unit. Crit Care Med 34(3):857-863

190. Vergidis P, Clancy CJ, Shields RK et al (2016) Intra-abdominal candidiasis: the importance of early source control and antifungal treatment. PLoS One 11(4):e0153247

191. Ballard N, Robley L, Barrett D et al (2006) Patients' recollections of therapeutic paralysis in the intensive care unit. Am J Crit Care 15(1):86-94 (quiz 5)

192. Horvath EE, Murray CK, Vaughan GM et al (2007) Fungal wound infection (not colonization) is independently associated with mortality in burn patients. Ann Surg 245(6):978-985

193. Murray CK, Loo FL, Hospenthal DR et al (2008) Incidence of systemic fungal infection and related mortality following severe burns. Burns 34(8):1108-1112

194. Baughman RP, Rhodes JC, Dohn MN et al (1992) Detection of cryptococcal antigen in bronchoalveolar lavage fluid: a prospective study of diagnostic utility. Am Rev Respir Dis 145(5):1226-1229

195. Ford N, Shubber Z, Jarvis JN et al (2018) CD4 cell count threshold for cryptococcal antigen screening of HIV-infected individuals: a systematic review and meta-analysis. Clin Infect Dis 66(2):S152-S159

196. Hage CA, Ribes JA, Wengenack NL et al (2011) A multicenter evaluation of tests for diagnosis of histoplasmosis. Clin Infect Dis 53(5):448-454

197. Clumeck N, Sonnet J, Taelman H et al (1984) Acquired immunodeficiency syndrome in African patients. N Engl J Med 310(8):492-497

198. Hajjeh RA, Conn LA, Stephens DS et al (1999) Cryptococcosis: population-based multistate active surveillance and risk factors in human immunodeficiency virus-infected persons. Cryptococca Active Surveillance Group. J Infect Dis 179(2):449-454

199. Maziarz EK, Perfect JR (2016) Cryptococcosis. Infect Dis Clin N Am 30(1):179-206

200. McCarthy KM, Morgan J, Wannemuehler KA et al (2006) Populationbased surveillance for cryptococcosis in an antiretroviral-naive South African province with a high HIV seroprevalence. AIDS 20(17):2199-2206

201. Husain S, Wagener MM, Singh N (2001) Cryptococcus neoformans infection in organ transplant recipients: variables influencing clinical characteristics and outcome. Emerg Infect Dis 7(3):375-381 
202. Pappas PG, Alexander BD, Andes DR et al (2010) Invasive fungal infections among organ transplant recipients: results of the TransplantAssociated Infection Surveillance Network (TRANSNET). Clin Infect Dis 50(8):1101-1111

203. Singh N, Gayowski T, Wagener MM et al (1997) Clinical spectrum of invasive cryptococcosis in liver transplant recipients receiving tacrolimus. Clin Transplant 11(1):66-70

204. Kontoyiannis DP, Marr KA, Park BJ et al (2010) Prospective surveillance for invasive fungal infections in hematopoietic stem cell transplant recipients, 2001-2006: overview of the Transplant-Associated Infection Surveillance Network (TRANSNET) Database. Clin Infect Dis 50(8):1091-1100

205. Nath DS, Kandaswamy R, Gruessner R et al (2005) Fungal infections in transplant recipients receiving alemtuzumab. Transplant Proc 37(2):934-936

206. Tsiodras S, Samonis G, Boumpas DT et al (2008) Fungal infections complicating tumor necrosis factor alpha blockade therapy. Mayo Clin Proc 83(2):181-194

207. Nsenga L, Kajjimu J, Olum R et al (2021) Cryptococcosis complicating diabetes mellitus: a scoping review. Ther Adv Infect Dis 8:20499361211014770

208. Wald A, Leisenring W, van Burik JA et al (1997) Epidemiology of Aspergillus infections in a large cohort of patients undergoing bone marrow transplantation. J Infect Dis 175(6):1459-1466

209. Mengoli C, Cruciani M, Barnes RA et al (2009) Use of PCR for diagnosis of invasive aspergillosis: systematic review and meta-analysis. Lancet Infect Dis 9(2):89-96

210. White PL, Bretagne S, Klingspor L et al (2010) Aspergillus PCR: one step closer to standardization. J Clin Microbiol 48(4):1231-1240

211. White PL, Wingard JR, Bretagne S et al (2015) Aspergillus polymerase chain reaction: systematic review of evidence for clinical use in comparison with antigen testing. Clin Infect Dis 61 (8):1293-1303

212. Meersseman W, Lagrou K, Maertens J et al (2007) Invasive aspergillosis in the intensive care unit. Clin Infect Dis 45(2):205-216

213. Barnes PD, Marr KA (2006) Aspergillosis: spectrum of disease, diagnosis, and treatment. Infect Dis Clin N Am. 20(3):545-561 (vi)

214. Gavalda J, Len O, San Juan R et al (2005) Risk factors for invasive aspergillosis in solid-organ transplant recipients: a case-control study. Clin Infect Dis 41(1):52-59

215. Fukuda T, Boeckh M, Carter RA et al (2003) Risks and outcomes of invasive fungal infections in recipients of allogeneic hematopoietic stem cell transplants after nonmyeloablative conditioning. Blood 102(3):827-833

216. Pagano L, Busca A, Candoni A et al (2017) Risk stratification for invasive fungal infections in patients with hematological malignancies: SEIFEM recommendations. Blood Rev 31(2):17-29

217. Baddley JW (2011) Clinical risk factors for invasive aspergillosis. Med Mycol 49(Suppl 1):S7-S12

218. Ruiz-Camps I, Aguilar-Company J (2021) Risk of infection associated with targeted therapies for solid organ and hematological malignancies. Ther Adv Infect Dis 8:2049936121989548

219. Cantan B, Luyt CE, Martin-Loeches I (2019) Influenza infections and emergent viral infections in intensive care unit. Semin Respir Crit Care Med 40(4):488-497

220. Legoff J, Zucman N, Lemiale $V$ et al (2019) Clinical significance of upper airway virus detection in critically ill hematology patients. Am J Respir Crit Care Med 199(4):518-528

221. Muscedere J, Ofner M, Kumar A et al (2013) The occurrence and impact of bacterial organisms complicating critical care illness associated with 2009 influenza A(H1N1) infection. Chest 144(1):39-47

222. van Someren GF, Juffermans NP, Bos LDJ et al (2018) Respiratory viruses in invasively ventilated critically ill patients-a prospective multicenter observational study. Crit Care Med 46(1):29-36

223. Aziz S, Arabi YM, Alhazzani W, et al (2020) Managing ICU surge during the COVID-19 crisis: rapid guidelines. Intensive Care Med 46(7):1303-1325

224. Wiersinga WJ, Rhodes A, Cheng AC et al (2020) Pathophysiology, transmission, diagnosis, and rreatment of Coronavirus Disease 2019 (COVID-19): a review. JAMA 324(8):782-793

225. Muthuri SG, Venkatesan S, Myles PR et al (2014) Effectiveness of neuraminidase inhibitors in reducing mortality in patients admitted to hospital with influenza A H1N1pdm09 virus infection: a meta-analysis of individual participant data. Lancet Respir Med 2(5):395-404

226. Alhazzani W, Moller MH, Arabi YM et al (2020) Surviving Sepsis Campaign: guidelines on the management of critically ill adults with Coronavirus Disease 2019 (COVID-19). Intensive Care Med 46(5):854-887

227. Tunkel AR, Glaser CA, Bloch KC et al (2008) The management of encephalitis: clinical practice guidelines by the Infectious Diseases Society of America. Clin Infect Dis 47(3):303-327

228. Uyeki TM, Bernstein HH, Bradley JS et al (2019) Clinical Practice Guidelines by the Infectious Diseases Society of America: 2018 Update on Diagnosis, Treatment, Chemoprophylaxis, and Institutional Outbreak Management of Seasonal Influenzaa. Clin Infect Dis 68(6):895-902

229. Lin GL, McGinley JP, Drysdale SB et al (2018) Epidemiology and immune pathogenesis of viral sepsis. Front Immunol 9:2147

230. Goncalves-Pereira J, Povoa P (2011) Antibiotics in critically ill patients: a systematic review of the pharmacokinetics of beta-lactams. Crit Care 15(5):R206

231. Mohd Hafiz AA, Staatz CE, Kirkpatrick CM et al (2012) Continuous infusion vs. bolus dosing: implications for beta-lactam antibiotics. Minerva Anestesiol 78(1):94-104

232. Roberts JA, Abdul-Aziz MH, Davis JS et al (2016) Continuous versus intermittent beta-lactam infusion in severe sepsis. A meta-analysis of individual patient data from randomized trials. Am J Respir Crit Care Med 194(6):681-691

233. Vardakas KZ, Voulgaris GL, Maliaros A et al (2018) Prolonged versus short-term intravenous infusion of antipseudomonal beta-lactams for patients with sepsis: a systematic review and meta-analysis of randomised trials. Lancet Infect Dis 18(1):108-120

234. De Waele JJ, Lipman J, Carlier M et al (2015) Subtleties in practical application of prolonged infusion of beta-lactam antibiotics. Int J Antimicrob Agents 45(5):461-463

235. Roberts JA, Paratz J, Paratz E et al (2007) Continuous infusion of beta-lactam antibiotics in severe infections: a review of its role. Int J Antimicrob Agents 30(1):11-18

236. Lipman J, Brett SJ, De Waele JJ et al (2019) A protocol for a phase 3 multicentre randomised controlled trial of continuous versus intermittent beta-lactam antibiotic infusion in critically ill patients with sepsis: BLING III. Crit Care Resusc 21(1):63-68

237. Roberts JA, Abdul-Aziz MH, Lipman J et al (2014) Individualised antibiotic dosing for patients who are critically ill: challenges and potential solutions. Lancet Infect Dis 14(6):498-509

238. Roberts JA, Paul SK, Akova M et al (2014) DALI: defining antibiotic levels in intensive care unit patients: are current beta-lactam antibiotic doses sufficient for critically ill patients? Clin Infect Dis 58(8):1072-1083

239. Veiga RP, Paiva JA (2018) Pharmacokinetics-pharmacodynamics issues relevant for the clinical use of beta-lactam antibiotics in critically ill patients. Crit Care 22(1):233

240. Nelson NR, Morbitzer KA, Jordan JD et al (2019) The impact of capping creatinine clearance on achieving therapeutic vancomycin concentrations in neurocritically ill patients with traumatic brain injury. Neurocrit Care 30(1):126-131

241. Gregoire N, Marchand S, Ferrandiere M et al (2019) Population pharmacokinetics of daptomycin in critically ill patients with various degrees of renal impairment. J Antimicrob Chemother 74(1):117-125

242. Ulldemolins M, Roberts JA, Rello J et al (2011) The effects of hypoalbuminaemia on optimizing antibacterial dosing in critically ill patients. Clin Pharmacokinet 50(2):99-110

243. Choi G, Gomersall CD, Tian Q et al (2009) Principles of antibacterial dosing in continuous renal replacement therapy. Crit Care Med 37(7):2268-2282

244. Roberts JA, Joynt G, Lee A, et al (2020) The effect of renal replacement therapy and antibiotic dose on antibiotic concentrations in critically ill patients: data from the multinational SMARRT Study. Clin Infect Dis 72(8):1369-1378

245. Bougle A, Dujardin O, Lepere V et al (2019) PHARMECMO: Therapeutic drug monitoring and adequacy of current dosing regimens of antibiotics in patients on Extracorporeal Life Support. Anaesth Crit Care Pain Med 38(5):493-497

246. Cheng V, Abdul-Aziz MH, Roberts JA et al (2019) Overcoming barriers to optimal drug dosing during ECMO in critically ill adult patients. Expert Opin Drug Metab Toxicol 15(2):103-112 
247. Guilhaumou R, Benaboud S, Bennis Y et al (2019) Optimization of the treatment with beta-lactam antibiotics in critically ill patientsguidelines from the French Society of Pharmacology and Therapeutics (Societe Francaise de Pharmacologie et Therapeutique-SFPT) and the French Society of Anaesthesia and Intensive Care Medicine (Societe Francaise d'Anesthesie et Reanimation-SFAR). Crit Care 23(1):104

248. Turner RB, Kojiro K, Shephard EA et al (2018) Review and validation of bayesian dose-optimizing software and equations for calculation of the vancomycin area under the curve in critically ill patients. Pharmacotherapy 38(12):1174-1183

249. Rybak M, Lomaestro B, Rotschafer JC et al (2009) Therapeutic monitoring of vancomycin in adult patients: a consensus review of the American Society of Health-System Pharmacists, the Infectious Diseases Society of America, and the Society of Infectious Diseases Pharmacists. Am J Health Syst Pharm 66(1):82-98

250. McKinnon PS, Paladino JA, Schentag JJ (2008) Evaluation of area under the inhibitory curve (AUIC) and time above the minimum inhibitory concentration ( $\mathrm{T}>\mathrm{MIC}$ ) as predictors of outcome for cefepime and ceftazidime in serious bacterial infections. Int J Antimicrob Agents 31(4):345-351

251. Rayner CR, Forrest A, Meagher AK et al (2003) Clinical pharmacodynamics of linezolid in seriously ill patients treated in a compassionate use programme. Clin Pharmacokinet 42(15):1411-1423

252. Rubino CM, Bhavnani SM, Forrest A et al (2012) Pharmacokinetics-pharmacodynamics of tigecycline in patients with community-acquired pneumonia. Antimicrob Agents Chemother 56(1):130-136

253. Wong G, Taccone F, Villois P et al (2020) beta-Lactam pharmacodynamics in Gram-negative bloodstream infections in the critically ill. J Antimicrob Chemother 75(2):429-433

254. Fleuren LM, Roggeveen LF, Guo T et al (2019) Clinically relevant pharmacokinetic knowledge on antibiotic dosing among intensive care professionals is insufficient: a cross-sectional study. Crit Care 23(1):185

255. Ehmann L, Zoller M, Minichmayr IK et al (2019) Development of a dosing algorithm for meropenem in critically ill patients based on a population pharmacokinetic/pharmacodynamic analysis. Int J Antimicrob Agents 54(3):309-317

256. Wong G, Briscoe S, McWhinney B et al (2018) Therapeutic drug monitoring of beta-lactam antibiotics in the critically ill: direct measurement of unbound drug concentrations to achieve appropriate drug exposures. J Antimicrob Chemother 73(11):3087-3094

257. Williams P, Beall G, Cotta MO et al (2020) Antimicrobial dosing in critical care: a pragmatic adult dosing nomogram. Int J Antimicrob Agents 55(2): 105837

258. Williams P, Cotta MO, Roberts JA (2019) Pharmacokinetics/Pharmacodynamics of beta-Lactams and Therapeutic Drug Monitoring: From Theory to Practical Issues in the Intensive Care Unit. Semin Respir Crit Care Med 40(4):476-487

259. Nation RL, Garonzik SM, Thamlikitkul V et al (2017) Dosing guidance for intravenous colistin in critically-ill patients. Clin Infect Dis 64(5):565-571

260. Roberts JA, Taccone FS, Udy AA et al (2011) Vancomycin dosing in critically ill patients: robust methods for improved continuous-infusion regimens. Antimicrob Agents Chemother 55(6):2704-2709

261. Sinnollareddy M, Peake SL, Roberts MS et al (2012) Using pharmacokinetics and pharmacodynamics to optimise dosing of antifungal agents in critically ill patients: a systematic review. Int J Antimicrob Agents 39(1):1-10

262. Jimenez MF, Marshall JC, International Sepsis F (2001) Source control in the management of sepsis. Intensive Care Med 27(Suppl 1):S49-62

263. Kim H, Chung SP, Choi SH et al (2019) Impact of timing to source control in patients with septic shock: a prospective multi-center observational study. J Crit Care 53:176-182

264. Martinez ML, Ferrer R, Torrents E et al (2017) Impact of Source Control in Patients With Severe Sepsis and Septic Shock. Crit Care Med 45(1):11-19

265. Azuhata T, Kinoshita K, Kawano D et al (2014) Time from admission to initiation of surgery for source control is a critical determinant of survival in patients with gastrointestinal perforation with associated septic shock. Crit Care 18(3):R87

266. Bloos F, Thomas-Ruddel D, Ruddel H et al (2014) Impact of compliance with infection management guidelines on outcome in patients with severe sepsis: a prospective observational multi-center study. Crit Care 18(2):R42

267. Buck DL, Vester-Andersen M, Moller MH (2013) Surgical delay is a critical determinant of survival in perforated peptic ulcer. Br J Surg 100(8):1045-1049

268. Chao WN, Tsai CF, Chang HR et al (2013) Impact of timing of surgery on outcome of Vibrio vulnificus-related necrotizing fasciitis. Am J Surg 206(1):32-39

269. Karvellas CJ, Abraldes JG, Zepeda-Gomez S et al (2016) The impact of delayed biliary decompression and anti-microbial therapy in 260 patients with cholangitis-associated septic shock. Aliment Pharmacol Ther 44(7):755-766

270. Moss RL, Musemeche CA, Kosloske AM (1996) Necrotizing fasciitis in children: prompt recognition and aggressive therapy improve survival. J Pediatr Surg 31(8):1142-1146

271. Wong $\mathrm{CH}$, Chang HC, Pasupathy S et al (2003) Necrotizing fasciitis: clinical presentation, microbiology, and determinants of mortality. J Bone Joint Surg Am 85(8):1454-1460

272. Solomkin JS, Mazuski JE, Bradley JS et al (2010) Diagnosis and management of complicated intra-abdominal infection in adults and children: guidelines by the Surgical Infection Society and the Infectious Diseases Society of America. Clin Infect Dis 50(2):133-164

273. Mermel LA, Allon M, Bouza E et al (2009) Clinical practice guidelines for the diagnosis and management of intravascular catheter-related infection: 2009 Update by the Infectious Diseases Society of America. Clin Infect Dis 49(1):1-45

274. Rijnders BJ, Peetermans WE, Verwaest C et al (2004) Watchful waiting versus immediate catheter removal in ICU patients with suspected catheter-related infection: a randomized trial. Intensive Care Med 30(6):1073-1080

275. Garnacho-Montero J, Aldabo-Pallas T, Palomar-Martinez M et al (2008) Risk factors and prognosis of catheter-related bloodstream infection in critically ill patients: a multicenter study. Intensive Care Med 34(12):2185-2193

276. Lorente L, Martin MM, Vidal P et al (2014) Should central venous catheter be systematically removed in patients with suspected catheter related infection? Crit Care 18(5):564

277. Tabah A, Bassetti M, Kollef MH et al (2020) Antimicrobial de-escalation in critically ill patients: a position statement from a task force of the European Society of Intensive Care Medicine (ESICM) and European Society of Clinical Microbiology and Infectious Diseases (ESCMID) Critically III Patients Study Group (ESGCIP). Intensive Care Med 46(2):245-265

278. Leone M, Bechis C, Baumstarck K et al (2014) De-escalation versus continuation of empirical antimicrobial treatment in severe sepsis: a multicenter non-blinded randomized noninferiority trial. Intensive Care Med 40(10):1399-1408

279. Tabah A, Cotta MO, Garnacho-Montero J et al (2016) A systematic review of the definitions, determinants, and clinical outcomes of antimicrobial de-escalation in the intensive care unit. Clin Infect Dis 62(8):1009-1017

280. De Bus L, Depuydt P, Steen J, et al (2020) Antimicrobial de-escalation in the critically ill patient and assessment of clinical cure: the DIANA study. Intensive Care Med 46(7):1404-1417

281. Fernandez-Lazaro Cl, Brown KA, Langford BJ et al (2019) Late-career physicians prescribe longer courses of antibiotics. Clin Infect Dis 69(9):1467-1475

282. Hanretty AM, Gallagher JC (2018) Shortened courses of antibiotics for bacterial infections: a systematic review of randomized controlled trials. Pharmacotherapy 38(6):674-687

283. Royer S, DeMerle KM, Dickson RP et al (2018) shorter versus longer courses of antibiotics for infection in hospitalized patients: a systematic review and meta-analysis. J Hosp Med 13(5):336-342

284. Spellberg B (2016) The new antibiotic mantra-"Shorter Is Better". JAMA Intern Med 176(9):1254-1255

285. Wald-Dickler N, Spellberg B (2019) Short-course antibiotic therapyreplacing constantine units with "Shorter Is Better." Clin Infect Dis 69(9):1476-1479

286. Chastre J, Wolff M, Fagon JY et al (2003) Comparison of 8 vs 15 days of antibiotic therapy for ventilator-associated pneumonia in adults: a randomized trial. JAMA 290(19):2588-2598 
287. Choudhury G, Mandal P, Singanayagam A et al (2011) Seven-day antibiotic courses have similar efficacy to prolonged courses in severe community-acquired pneumonia-a propensity-adjusted analysis. Clin Microbiol Infect 17(12):1852-1858

288. Kalil AC, Metersky ML, Klompas M et al (2016) Management of adults with hospital-acquired and ventilator-associated pneumonia: 2016 clinical practice guidelines by the infectious diseases society of America and the American Thoracic Society. Clin Infect Dis 63(5):e61-e111

289. Vaughn VM, Flanders SA, Snyder A et al (2019) Excess antibiotic treatment duration and adverse events in patients hospitalized with pneumonia: a multihospital cohort study. Ann Intern Med 171(3):153-163

290. Eliakim-Raz N, Yahav D, Paul M et al (2013) Duration of antibiotic treatment for acute pyelonephritis and septic urinary tract infection -7 days or less versus longer treatment: systematic review and meta-analysis of randomized controlled trials. J Antimicrob Chemother 68(10):2183-2191

291. Runyon BA, McHutchison JG, Antillon MR et al (1991) Short-course versus long-course antibiotic treatment of spontaneous bacterial peritonitis. A randomized controlled study of 100 patients. Gastroenterology 100(6):1737-1742

292. Yahav D, Franceschini E, Koppel F et al (2019) Seven versus 14 days of antibiotic therapy for uncomplicated gram-negative bacteremia: a noninferiority randomized controlled Trial. Clin Infect Dis 69(7):1091-1098

293. Sawyer RG, Claridge JA, Nathens AB et al (2015) Trial of short-course antimicrobial therapy for intraabdominal infection. N Engl J Med 372(21):1996-2005

294. Corona A, Bertolini G, Ricotta AM et al (2003) Variability of treatment duration for bacteraemia in the critically ill: a multinational survey. J Antimicrob Chemother 52(5):849-852

295. Burnham JP, Olsen MA, Stwalley D et al (2018) Infectious diseases consultation reduces 30-day and 1-year all-cause mortality for multidrugresistant organism infections. Open Forum Infect Dis 5(3):ofy06

296. Macheda G, Dyar OJ, Luc A et al (2018) Are infection specialists recommending short antibiotic treatment durations? An ESCMID international cross-sectional survey. J Antimicrob Chemother 73(4):1084-1090

297. Madaline T, Wadskier Montagne F, Eisenberg R et al (2019) Early infectious disease consultation is associated with lower mortality in patients with severe sepsis or septic shock who complete the 3-hour sepsis treatment bundle. Open Forum Infect Dis 6(10):ofz408

298. Schmitt S, McQuillen DP, Nahass R et al (2014) Infectious diseases specialty intervention is associated with decreased mortality and lower healthcare costs. Clin Infect Dis 58(1):22-28

299. Turner RB, Valcarlos E, Won R et al (2016) Impact of infectious diseases consultation on clinical outcomes of patients with staphylococcus aureus bacteremia in a community health system. Antimicrob Agents Chemother 60(10):5682-5687

300. Viale P, Tedeschi S, Scudeller L et al (2017) Infectious diseases team for the early management of severe sepsis and septic shock in the emergency department. Clin Infect Dis 65(8):1253-1259

301. Pugh R, Grant C, Cooke RP et al (2015) Short-course versus prolongedcourse antibiotic therapy for hospital-acquired pneumonia in critically ill adults. Cochrane Database Syst Rev 8:CD007577

302. Havey TC, Fowler RA, Daneman N (2011) Duration of antibiotic therapy for bacteremia: a systematic review and meta-analysis. Crit Care 15(6):R267

303. Dimopoulos G, Matthaiou DK, Karageorgopoulos DE et al (2008) Shortversus long-course antibacterial therapy for community-acquired pneumonia: a meta-analysis. Drugs 68(13):1841-1854

304. Tansarli GS, Andreatos N, Pliakos EE, et al (2019) a systematic review and meta-analysis of antibiotic treatment duration for bacteremia due to enterobacteriaceae. Antimicrob Agents Chemother 63(5):e02495-18

305. Montravers P, Tubach F, Lescot T et al (2018) Short-course antibiotic therapy for critically ill patients treated for postoperative intra-abdominal infection: the DURAPOP randomised clinical trial. Intensive Care Med 44(3):300-310

306. Mazuski JE, Sawyer RG, Nathens AB et al (2002) The Surgical Infection Society guidelines on antimicrobial therapy for intra-abdominal infections: evidence for the recommendations. Surg Infect (Larchmt) 3(3):175-233

307. van Engelen TSR, Wiersinga WJ, Scicluna BP et al (2018) Biomarkers in Sepsis. Crit Care Clin 34(1):139-152
308. Annane D, Maxime V, Faller JP, et al (2013) Procalcitonin levels to guide antibiotic therapy in adults with non-microbiologically proven apparent severe sepsis: a randomised controlled trial. BMJ Open 3(2):e002186

309. Bloos F, Trips E, Nierhaus A et al (2016) Effect of Sodium Selenite Administration and Procalcitonin-Guided Therapy on Mortality in Patients With Severe Sepsis or Septic Shock: A Randomized Clinical Trial. JAMA Intern Med 176(9):1266-1276

310. Bouadma L, Luyt CE, Tubach F et al (2010) Use of procalcitonin to reduce patients' exposure to antibiotics in intensive care units (PRORATA trial): a multicentre randomised controlled trial. Lancet 375(9713):463-474

311. de Jong E, van Oers JA, Beishuizen A et al (2016) Efficacy and safety of procalcitonin guidance in reducing the duration of antibiotic treatment in critically ill patients: a randomised, controlled, open-label trial. Lancet Infect Dis 16(7):819-827

312. Deliberato RO, Marra AR, Sanches PR et al (2013) Clinical and economic impact of procalcitonin to shorten antimicrobial therapy in septic patients with proven bacterial infection in an intensive care setting. Diagn Microbiol Infect Dis 76(3):266-271

313. Hochreiter M, Kohler T, Schweiger AM et al (2009) Procalcitonin to guide duration of antibiotic therapy in intensive care patients: a randomized prospective controlled trial. Crit Care 13(3):R83

314. Liu BH, Li HF, Lei Y et al (2013) Clinical significance of dynamic monitoring of procalcitonin in guiding the use of antibiotics in patients with sepsis in ICU. Zhonghua Wei Zhong Bing Ji Jiu Yi Xue 25(11):690-693

315. Nobre V, Harbarth S, Graf JD et al (2008) Use of procalcitonin to shorten antibiotic treatment duration in septic patients: a randomized trial. Am J Respir Crit Care Med 177(5):498-505

316. Oliveira CF, Botoni FA, Oliveira CR et al (2013) Procalcitonin versus C-reactive protein for guiding antibiotic therapy in sepsis: a randomized trial. Crit Care Med 41(10):2336-2343

317. Qu R, Ji Y, Ling Y et al (2012) Procalcitonin is a good tool to guide duration of antibiotic therapy in patients with severe acute pancreatitis. A randomized prospective single-center controlled trial. Saudi Med J 33(4):382-387

318. Schroeder S, Hochreiter M, Koehler T et al (2009) Procalcitonin (PCT)guided algorithm reduces length of antibiotic treatment in surgical intensive care patients with severe sepsis: results of a prospective randomized study. Langenbecks Arch Surg 394(2):221-226

319. Shehabi Y, Sterba M, Garrett PM et al (2014) Procalcitonin algorithm in critically ill adults with undifferentiated infection or suspected sepsis. A randomized controlled trial. Am J Respir Crit Care Med 190(10):1102-1110

320. Stolz D, Smyrnios N, Eggimann P et al (2009) Procalcitonin for reduced antibiotic exposure in ventilator-associated pneumonia: a randomised study. Eur Respir J 34(6):1364-1375

321. Xu XL, Yan FD, Yu JQ et al (2017) Efficacy and safety of procalcitonin guidance in reducing the duration of antibiotic treatment of sepsis patients. Zhonghua Yi Xue Za Zhi 97(5):343-346

322. Arulkumaran N, Khpal M, Tam K et al (2020) Effect of antibiotic discontinuation strategies on mortality and infectious complications in critically ill septic patients: a meta-analysis and trial sequential analysis. Crit Care Med 48(5):757-764

323. Collins CD, Brockhaus K, Sim T et al (2019) Analysis to determine cost-effectiveness of procalcitonin-guided antibiotic use in adult patients with suspected bacterial infection and sepsis. Am J Health Syst Pharm 76(16):1219-1225

324. Lewis SR, Pritchard MW, Evans DJ et al (2018) Colloids versus crystalloids for fluid resuscitation in critically ill people. Cochrane Database Syst Rev 8:CD000567

325. Awad S, Allison SP, Lobo DN (2008) The history of 0.9\% saline. Clin Nutr 27(2):179-188

326. Chowdhury AH, Cox EF, Francis ST et al (2012) A randomized, controlled, double-blind crossover study on the effects of $2-\mathrm{L}$ infusions of $0.9 \%$ saline and plasma-lyte(R) 148 on renal blood flow velocity and renal cortical tissue perfusion in healthy volunteers. Ann Surg 256(1):18-24

327. Kellum JA (2002) Fluid resuscitation and hyperchloremic acidosis in experimental sepsis: improved short-term survival and acidbase balance with Hextend compared with saline. Crit Care Med 30(2):300-305 
328. Kellum JA, Song M, Almasri E (2006) Hyperchloremic acidosis increases circulating inflammatory molecules in experimental sepsis. Chest 130(4):962-967

329. Waters JH, Gottlieb A, Schoenwald P et al (2001) Normal saline versus lactated Ringer's solution for intraoperative fluid management in patients undergoing abdominal aortic aneurysm repair: an outcome study. Anesth Analg 93(4):817-822

330. Williams EL, Hildebrand KL, McCormick SA et al (1999) The effect of intravenous lactated Ringer's solution versus $0.9 \%$ sodium chloride solution on serum osmolality in human volunteers. Anesth Analg 88(5):999-1003

331. Rochwerg B, Alhazzani W, Sindi A et al (2014) Fluid resuscitation in sepsis: a systematic review and network meta-analysis. Ann Intern Med 161(5):347-355

332. Young P, Bailey M, Beasley R et al (2015) Effect of a buffered crystalloid solution vs saline on acute kidney injury among patients in the intensive care unit: the SPLIT Randomized Clinical Trial. JAMA 314(16):1701-1710

333. Semler MW, Wanderer JP, Ehrenfeld JM et al (2017) Balanced crystalloids versus saline in the intensive care unit. The SALT Randomized Trial. Am J Respir Crit Care Med 195(10):1362-1372

334. Semler MW, Self WH, Wanderer JP et al (2018) Balanced crystalloids versus saline in critically ill adults. N Engl J Med 378(9):829-839

335. Brown RM, Wang L, Coston TD et al (2019) Balanced crystalloids versus saline in sepsis. A secondary analysis of the SMART clinical trial. Am J Respir Crit Care Med 200(12):1487-1495

336. Myburgh J (2018) Patient-Centered Outcomes and Resuscitation Fluids. N Engl J Med 378(9):862-863

337. Zampieri FG, Azevedo LCP, Correa TD et al (2017) Study protocol for the Balanced Solution versus Saline in Intensive Care Study (BaSICS): a factorial randomised trial. Crit Care Resusc 19(2):175-182

338. Institute G (2020) Plasma-Lyte $148^{\circledR}$ versUs Saline Study (PLUS).: ClinicalTrials.gov. Available from: https://clinicaltrials.gov/ct2/show/NCT02 721654

339. Caironi P, Tognoni G, Gattinoni L (2014) Albumin replacement in severe sepsis or septic shock. N Engl J Med 371(1):84

340. Martin GS, Bassett P (2019) Crystalloids vs. colloids for fluid resuscitation in the Intensive Care Unit: a systematic review and meta-analysis. J Crit Care 50:144-154

341. Park CHL, de Almeida JP, de Oliveira GQ et al (2019) Lactated ringer's versus $4 \%$ albumin on lactated ringer's in early sepsis therapy in cancer patients: a pilot single-center randomized trial. Crit Care Med 47(10):e798-e805

342. Kakaei FHS, Asheghvatan A, Zarrintan S, Asvadi T, Beheshtirouy S, Mohajer A (2017) Albumin as a resuscitative fluid in patients with severe sepsis: a randomized clinical trial. Adv Biosci Clin Med 5(4):9-16

343. Haase N, Perner A, Hennings LI et al (2013) Hydroxyethyl starch $130 / 0.38-0.45$ versus crystalloid or albumin in patients with sepsis: systematic review with meta-analysis and trial sequential analysis. BMJ 346:f839

344. Annane D, Siami S, Jaber S et al (2013) Effects of fluid resuscitation with colloids vs crystalloids on mortality in critically ill patients presenting with hypovolemic shock: the CRISTAL randomized trial. JAMA 310(17):1809-1817

345. Rochwerg B, Alhazzani W, Gibson A et al (2015) Fluid type and the use of renal replacement therapy in sepsis: a systematic review and network meta-analysis. Intensive Care Med 41(9):1561-1571

346. Moeller C, Fleischmann C, Thomas-Rueddel D et al (2016) How safe is gelatin? A systematic review and meta-analysis of gelatin-containing plasma expanders vs crystalloids and albumin. J Crit Care 35:75-83

347. Avni T, Lador A, Lev $S$ et al (2015) Vasopressors for the treatment of septic shock: systematic review and meta-analysis. PLoS One 10(8):e0129305

348. Regnier B, Safran D, Carlet J et al (1979) Comparative haemodynamic effects of dopamine and dobutamine in septic shock. Intensive Care Med 5(3):115-120

349. De Backer D, Creteur J, Silva E et al (2003) Effects of dopamine, norepinephrine, and epinephrine on the splanchnic circulation in septic shock: which is best? Crit Care Med 31(6):1659-1667
350. Cui J, Wei X, Lv H et al (2019) The clinical efficacy of intravenous IgMenriched immunoglobulin (pentaglobin) in sepsis or septic shock: a meta-analysis with trial sequential analysis. Ann Intensive Care 9(1):27

351. Myburgh JA, Higgins A, Jovanovska A et al (2008) A comparison of epinephrine and norepinephrine in critically ill patients. Intensive Care Med 34(12):2226-2234

352. Holmes CL, Patel BM, Russell JA et al (2001) Physiology of vasopressin relevant to management of septic shock. Chest 120(3):989-1002

353. Landry DW, Levin HR, Gallant EM et al (1997) Vasopressin deficiency contributes to the vasodilation of septic shock. Circulation 95(5):1122-1125

354. Gordon AC, Mason AJ, Thirunavukkarasu N et al (2016) Effect of early vasopressin vs norepinephrine on kidney failure in patients with septic shock: the VANISH randomized clinical trial. JAMA 316(5):509-518

355. Dunser MW, Mayr AJ, Tur A et al (2003) Ischemic skin lesions as a complication of continuous vasopressin infusion in catecholamineresistant vasodilatory shock: incidence and risk factors. Crit Care Med 31(5):1394-1398

356. Russell JA, Walley KR, Singer J et al (2008) Vasopressin versus norepinephrine infusion in patients with septic shock. N Engl J Med 358(9):877-887

357. Ukor IF, Walley KR (2019) Vasopressin in Vasodilatory Shock. Crit Care Clin 35(2):247-261

358. Mclntyre WF, Um KJ, Alhazzani W et al (2018) Association of vasopressin plus catecholamine vasopressors vs catecholamines alone with atrial fibrillation in patients with distributive shock: a systematic review and meta-analysis. JAMA 319(18):1889-1900

359. Nagendran M, Russell JA, Walley KR et al (2019) Vasopressin in septic shock: an individual patient data meta-analysis of randomised controlled trials. Intensive Care Med 45(6):844-855

360. Gamper G, Havel C, Arrich J et al (2016) Vasopressors for hypotensive shock. Cochrane Database Syst Rev 2:CD0037709

361. Akinaga J, Lima V, Kiguti LR et al (2013) Differential phosphorylation, desensitization, and internalization of alpha1A-adrenoceptors activated by norepinephrine and oxymetazoline. Mol Pharmacol 83(4):870-881

362. Belletti A, Benedetto U, Biondi-Zoccai G et al (2017) The effect of vasoactive drugs on mortality in patients with severe sepsis and septic shock. A network meta-analysis of randomized trials. J Crit Care 37:91-98

363. Russell JA, Vincent JL, Kjolbye AL et al (2017) Selepressin, a novel selective vasopressin $\mathrm{V} 1 \mathrm{~A}$ agonist, is an effective substitute for norepinephrine in a phase lla randomized, placebo-controlled trial in septic shock patients. Crit Care 21(1):213

364. Laterre PF, Berry SM, Blemings A, et al (2019) Effect of selepressin vs placebo on ventilator- and vasopressor-free days in patients with septic shock: the SEPSIS-ACT randomized clinical trial. JAMA 322(15):1476-1485

365. Chawla LS, Busse L, Brasha-Mitchell E et al (2014) Intravenous angiotensin II for the treatment of high-output shock (ATHOS trial): a pilot study. Crit Care 18(5):534

366. Khanna A, English SW, Wang XS et al (2017) Angiotensin II for the treatment of vasodilatory shock. N Engl J Med 377(5):419-430

367. Liu ZM, Chen J, Kou Q et al (2018) Terlipressin versus norepinephrine as infusion in patients with septic shock: a multicentre, randomised, double-blinded trial. Intensive Care Med 44(11):1816-1825

368. Walley KR (2018) Sepsis-induced myocardial dysfunction. Curr Opin Crit Care 24(4):292-299

369. Cunha-Goncalves D, Perez-de-Sa V, Larsson A et al (2009) Inotropic support during experimental endotoxemic shock: part II. A comparison of levosimendan with dobutamine. Anesth Analg 109(5):1576-1583

370. Dubin A, Lattanzio B, Gatti L (2017) The spectrum of cardiovascular effects of dobutamine-from healthy subjects to septic shock patients. Rev Bras Ter Intensiva 29(4):490-498

371. Wilkman E, Kaukonen KM, Pettila V et al (2013) Association between inotrope treatment and 90-day mortality in patients with septic shock. Acta Anaesthesiol Scand 57(4):431-442

372. Dunser MW, Festic E, Dondorp A et al (2012) Recommendations for sepsis management in resource-limited settings. Intensive Care Med 38(4):557-574 
373. Gordon AC, Perkins GD, Singer M et al (2016) Levosimendan for the prevention of acute organ dysfunction in sepsis. N Engl J Med 375(17):1638-1648

374. Bhattacharjee S, Soni KD, Maitra S et al (2017) Levosimendan does not provide mortality benefit over dobutamine in adult patients with septic shock: a meta-analysis of randomized controlled trials. J Clin Anesth 39:67-72

375. Araghi A, Bander JJ, Guzman JA (2006) Arterial blood pressure monitoring in overweight critically ill patients: invasive or noninvasive? Crit Care 10(2):R64

376. Bur A, Hirschl MM, Herkner H et al (2000) Accuracy of oscillometric blood pressure measurement according to the relation between cuff size and upper-arm circumference in critically ill patients. Crit Care Med 28(2):371-376

377. Kaur B, Kaur S, Yaddanapudi LN et al (2019) Comparison between invasive and noninvasive blood pressure measurements in critically ill patients receiving inotropes. Blood Press Monit 24(1):24-29

378. Lehman LW, Saeed M, Talmor D et al (2013) Methods of blood pressure measurement in the ICU. Crit Care Med 41(1):34-40

379. Riley LE, Chen GJ, Latham HE (2017) Comparison of noninvasive blood pressure monitoring with invasive arterial pressure monitoring in medical ICU patients with septic shock. Blood Press Monit 22(4):202-207

380. Vincent J (2019) Arterial, central venous, and pulmonary artery catheters. In: JE P (ed) Critical care medicine: principles and diagnosis and management in the adult, 5th edn. Elsevier, Philadelphia, pp 40-49

381. Scheer B, Perel A, Pfeiffer UJ (2002) Clinical review: complications and risk factors of peripheral arterial catheters used for haemodynamic monitoring in anaesthesia and intensive care medicine. Crit Care 6(3):199-204

382. Bhattacharjee S, Maitra S, Baidya DK (2018) Comparison between ultrasound guided technique and digital palpation technique for radial artery cannulation in adult patients: an updated meta-analysis of randomized controlled trials. J Clin Anesth 47:54-59

383. Gu WJ, Wu XD, Wang F et al (2016) Ultrasound guidance facilitates radial artery catheterization: a meta-analysis with trial sequential analysis of randomized controlled trials. Chest 149(1):166-179

384. O'Horo JC, Maki DG, Krupp AE et al (2014) Arterial catheters as a source of bloodstream infection: a systematic review and meta-analysis. Crit Care Med 42(6):1334-1339

385. Delaney A, Finnis M, Bellomo R et al (2020) Initiation of vasopressor infusions via peripheral versus central access in patients with early septic shock: a retrospective cohort study. Emerg Med Australas 32(2):210-219

386. Ricard JD, Salomon L, Boyer A et al (2013) Central or peripheral catheters for initial venous access of ICU patients: a randomized controlled trial. Crit Care Med 41(9):2108-2115

387. Cardenas-Garcia J, Schaub KF, Belchikov YG et al (2015) Safety of peripheral intravenous administration of vasoactive medication. J Hosp Med 10(9):581-585

388. Tian DH, Smyth C, Keijzers G et al (2020) Safety of peripheral administration of vasopressor medications: a systematic review. Emerg Med Australas 32(2):220-227

389. Loubani OM, Green RS (2015) A systematic review of extravasation and local tissue injury from administration of vasopressors through peripheral intravenous catheters and central venous catheters. J Crit Care 30(3):653e9-17

390. Beck V, Chateau D, Bryson GL et al (2014) Timing of vasopressor initiation and mortality in septic shock: a cohort study. Crit Care 18(3):R97

391. Black LP, Puskarich MA, Smotherman C et al (2020) Time to vasopressor initiation and organ failure progression in early septic shock. J Am Coll Emerg Physicians Open 1(3):222-230

392. Edaigbini SAAM, Delia IZ, Ibrahim A, Okwunodulo O, AlegbejoOlarinoye M (2017) Clinical competence with central venous lines by resident doctors in a Nigerian teaching hospital. Sub-Saharan Afr J Med 4:47-51

393. Rivers E, Nguyen B, Havstad S et al (2001) Early goal-directed therapy in the treatment of severe sepsis and septic shock. N Engl J Med 345(19):1368-1377

394. Alphonsus CS, Rodseth RN (2014) The endothelial glycocalyx: a review of the vascular barrier. Anaesthesia 69(7):777-784
395. Boyd JH, Forbes J, Nakada TA et al (2011) Fluid resuscitation in septic shock: a positive fluid balance and elevated central venous pressure are associated with increased mortality. Crit Care Med 39(2):259-265

396. Marik PE, Linde-Zwirble WT, Bittner EA et al (2017) Fluid administration in severe sepsis and septic shock, patterns and outcomes: an analysis of a large national database. Intensive Care Med 43(5):625-632

397. Chen C, Kollef MH (2015) Targeted fluid minimization following initial resuscitation in septic shock: a pilot study. Chest 148(6):1462-1469

398. Corl KA, Prodromou M, Merchant RC et al (2019) The restrictive IV Fluid trial in severe sepsis and septic shock (RIFTS): a randomized pilot study. Crit Care Med 47(7):951-959

399. Hjortrup PB, Haase N, Bundgaard $\mathrm{H}$ et al (2016) Restricting volumes of resuscitation fluid in adults with septic shock after initial management: the CLASSIC randomised, parallel-group, multicentre feasibility trial. Intensive Care Med 42(11):1695-1705

400. Macdonald SPJ, Keijzers G, Taylor DM et al (2018) Restricted fluid resuscitation in suspected sepsis associated hypotension (REFRESH): a pilot randomised controlled trial. Intensive Care Med 44(12):2070-2078

401. Semler MW, Janz DR, Casey JD, et al (2019) Conservative fluid management after sepsis resuscitation: a pilot randomized trial. J Intensive Care Med 35(12):1374-1382. https://doi.org/10.1177/0885066618823183

402. Meyhoff TS, Hjortrup PB, Moller MH et al (2019) Conservative vs liberal fluid therapy in septic shock (CLASSIC) trial-Protocol and statistical analysis plan. Acta Anaesthesiol Scand 63(9):1262-1271

403. Self WH, Semler MW, Bellomo R et al (2018) Liberal versus restrictive intravenous fluid therapy for early septic shock: rationale for a randomized trial. Ann Emerg Med 72(4):457-466

404. Girardis M, Busani S, Damiani E et al (2016) Effect of Conservative vs conventional oxygen therapy on mortality among patients in an intensive care unit: the oxygen-ICU randomized clinical trial. JAMA 316(15):1583-1589

405. Investigators I-R, the A, New Zealand Intensive Care Society Clinical Trials $G$ et al (2020) Conservative Oxygen Therapy during Mechanical Ventilation in the ICU. N Engl J Med 382(11):989-998

406. Panwar R, Hardie M, Bellomo R et al (2016) Conservative versus liberal oxygenation targets for mechanically ventilated patients. A pilot multicenter randomized controlled trial. Am J Respir Crit Care Med 193(1):43-51

407. Chu DK, Kim LH, Young PJ et al (2018) Mortality and morbidity in acutely ill adults treated with liberal versus conservative oxygen therapy (IOTA): a systematic review and meta-analysis. Lancet 391(10131):1693-1705

408. Young P, Mackle D, Bellomo R et al (2020) Conservative oxygen therapy for mechanically ventilated adults with sepsis: a post hoc analysis of data from the intensive care unit randomized trial comparing two approaches to oxygen therapy (ICU-ROX). Intensive Care Med 46(1):17-26

409. Barrot L, Asfar P, Mauny F et al (2020) Liberal or conservative oxygen therapy for acute respiratory distress syndrome. N Engl J Med 382(11):999-1008

410. Mauri T, Turrini C, Eronia N et al (2017) Physiologic effects of high-flow nasal cannula in acute hypoxemic respiratory failure. Am J Respir Crit Care Med 195(9):1207-1215

411. Frat JP, Thille AW, Mercat A et al (2015) High-flow oxygen through nasal cannula in acute hypoxemic respiratory failure. $N$ Engl J Med 372(23):2185-2196

412. Ni YN, Luo J, Yu H et al (2018) The effect of high-flow nasal cannula in reducing the mortality and the rate of endotracheal intubation when used before mechanical ventilation compared with conventional oxygen therapy and noninvasive positive pressure ventilation. A systematic review and meta-analysis. Am J Emerg Med 36(2):226-233

413. Ou X, Hua Y, Liu J et al (2017) Effect of high-flow nasal cannula oxygen therapy in adults with acute hypoxemic respiratory failure: a metaanalysis of randomized controlled trials. CMAJ 189(7):E260-E267

414. Rochwerg B, Granton D, Wang DX et al (2019) High-flow nasal cannula compared with conventional oxygen therapy for acute hypoxemic respiratory failure: author's reply. Intensive Care Med 45(8):1171

415. Demoule A, Chevret S, Carlucci A et al (2016) Changing use of noninvasive ventilation in critically ill patients: trends over 15 years in francophone countries. Intensive Care Med 42(1):82-92 
416. Demoule A, Girou E, Richard JC et al (2006) Benefits and risks of success or failure of noninvasive ventilation. Intensive Care Med 32(11):1756-1765

417. Bellani G, Laffey JG, Pham T et al (2017) Noninvasive ventilation of patients with acute respiratory distress syndrome. Insights from the LUNG SAFE Study. Am J Respir Crit Care Med 195(1):67-77

418. Antonelli M, Conti G, Rocco M et al (1998) A comparison of noninvasive positive-pressure ventilation and conventional mechanical ventilation in patients with acute respiratory failure. N Engl J Med 339(7):429-435

419. Honrubia T, Garcia Lopez FJ, Franco N et al (2005) Noninvasive vs conventional mechanical ventilation in acute respiratory failure: a multicenter, randomized controlled trial. Chest 128(6):3916-3924

420. Belenguer-Muncharaz A, Cubedo-Bort M, Blasco-Asensio D et al (2017) Non-invasive ventilation versus invasive mechanical ventilation in patients with hypoxemic acute respiratory failure in an Intensive Care Unit. A randomized controlled study. Minerva Pneumologica 56:1-10

421. Tonelli R, Fantini R, Tabbi L et al (2020) Early inspiratory effort assessment by esophageal manometry predicts noninvasive ventilation outcome in de novo respiratory failure. A pilot study. Am J Respir Crit Care Med 202(4):558-567

422. Bernard GR, Artigas A, Brigham KL et al (1994) The American-European Consensus Conference on ARDS definitions, mechanisms, relevant outcomes, and clinical trial coordination. Am J Respir Crit Care Med 149(3 Pt 1):818-824

423. Force ADT, Ranieri VM, Rubenfeld GD et al (2012) Acute respiratory distress syndrome: the Berlin Definition. JAMA 307(23):2526-2533

424. Brower RG, Matthay MA, Acute Respiratory Distress Syndrome N et al (2000) Ventilation with lower tidal volumes as compared with traditional tidal volumes for acute lung injury and the acute respiratory distress syndrome. N Engl J Med 342(18):1301-1308

425. Amato MB, Barbas CS, Medeiros DM et al (1998) Effect of a protectiveventilation strategy on mortality in the acute respiratory distress syndrome. N Engl J Med 338(6):347-354

426. Brochard L, Roudot-Thoraval F, Roupie E et al (1998) Tidal volume reduction for prevention of ventilator-induced lung injury in acute respiratory distress syndrome. The Multicenter Trail Group on Tidal Volume reduction in ARDS. Am J Respir Crit Care Med 158(6):1831-1838

427. Brower RG, Shanholtz CB, Fessler HE et al (1999) Prospective, randomized, controlled clinical trial comparing traditional versus reduced tidal volume ventilation in acute respiratory distress syndrome patients. Crit Care Med 27(8):1492-1498

428. Eichacker PQ, Gerstenberger EP, Banks SM et al (2002) Meta-analysis of acute lung injury and acute respiratory distress syndrome trials testing low tidal volumes. Am J Respir Crit Care Med 166(11):1510-1514

429. Marini JJ, Gattinoni L (2004) Ventilatory management of acute respiratory distress syndrome: a consensus of two. Crit Care Med 32(1):250-255

430. Tobin MJ (2000) Culmination of an era in research on the acute respiratory distress syndrome. N Engl J Med 342(18):1360-1361

431. Hager DN, Krishnan JA, Hayden DL et al (2005) Tidal volume reduction in patients with acute lung injury when plateau pressures are not high. Am J Respir Crit Care Med 172(10):1241-1245

432. Checkley W, Brower R, Korpak A et al (2008) Effects of a clinical trial on mechanical ventilation practices in patients with acute lung injury. Am J Respir Crit Care Med 177(11):1215-1222

433. Amato MB, Meade MO, Slutsky AS et al (2015) Driving pressure and survival in the acute respiratory distress syndrome. $\mathrm{N}$ Engl J Med 372(8):747-755

434. Papazian L, Aubron C, Brochard L et al (2019) Formal guidelines: management of acute respiratory distress syndrome. Ann Intensive Care 9(1):69

435. Laffey JG, Bellani G, Pham T et al (2016) Potentially modifiable factors contributing to outcome from acute respiratory distress syndrome: the LUNG SAFE study. Intensive Care Med 42(12):1865-1876

436. Villar J, Martin-Rodriguez C, Dominguez-Berrot AM et al (2017) A quantile analysis of plateau and driving pressures: effects on mortality in patients with acute respiratory distress syndrome receiving lungprotective ventilation. Crit Care Med 45(5):843-850

437. Hodgson CL, Cooper DJ, Arabi Y et al (2019) Maximal recruitment open lung ventilation in acute respiratory distress syndrome (PHARLAP). A phase II, multicenter randomized controlled clinical trial. Am J Respir Crit Care Med 200(11):1363-1372

438. Cavalcanti AB, Suzumura EA, Writing Group for the Alveolar Recruitment for Acute Respiratory Distress Syndrome Trial I et al (2017) Effect of lung recruitment and titrated positive end-expiratory pressure (PEEP) vs low PEEP on mortality in patients with acute respiratory distress syndrome: a randomized clinical trial. JAMA 318(14):1335-1345

439. Brower RG, Lanken PN, MacIntyre N et al (2004) Higher versus lower positive end-expiratory pressures in patients with the acute respiratory distress syndrome. N Engl J Med 351(4):327-336

440. Meade MO, Cook DJ, Guyatt GH et al (2008) Ventilation strategy using low tidal volumes, recruitment maneuvers, and high positive endexpiratory pressure for acute lung injury and acute respiratory distress syndrome: a randomized controlled trial. JAMA 299(6):637-645

441. Mercat A, Richard JC, Vielle B et al (2008) Positive end-expiratory pressure setting in adults with acute lung injury and acute respiratory distress syndrome: a randomized controlled trial. JAMA 299(6):646-655

442. Kacmarek RM, Villar J, Sulemanji D et al (2016) Open lung approach for the acute respiratory distress syndrome: a pilot randomized. controlled trial. Crit Care Med 44(1):32-42

443. Briel M, Meade M, Mercat A et al (2010) Higher vs lower positive end-expiratory pressure in patients with acute lung injury and acute respiratory distress syndrome: systematic review and meta-analysis. JAMA 303(9):865-873

444. Goligher EC, Kavanagh BP, Rubenfeld GD et al (2014) Oxygenation response to positive end-expiratory pressure predicts mortality in acute respiratory distress syndrome. A secondary analysis of the LOVS and ExPress trials. Am J Respir Crit Care Med 190(1):70-76

445. Amato MB, Barbas CS, Medeiros DM et al (1995) Beneficial effects of the "open lung approach" with low distending pressures in acute respiratory distress syndrome. A prospective randomized study on mechanical ventilation. Am J Respir Crit Care Med 152(6 Pt 1):1835-1846

446. Gattinoni L, Caironi P, Cressoni M et al (2006) Lung recruitment in patients with the acute respiratory distress syndrome. N Engl J Med 354(17):1775-1786

447. Beitler JR, Sarge T, Banner-Goodspeed VM et al (2019) Effect of titrating positive end-expiratory pressure (PEEP) with an esophageal pressure-guided strategy vs an empirical high PEEP-Fio2 strategy on death and days free from mechanical ventilation among patients with acute respiratory distress syndrome: a randomized clinical trial. JAMA 321(9):846-857

448. Talmor D, Sarge T, Malhotra A et al (2008) Mechanical ventilation guided by esophageal pressure in acute lung injury. $\mathrm{N}$ Engl J Med 359(20):2095-2104

449. Turbil E, Galerneau LM, Terzi N et al (2019) Positive-end expiratory pressure titration and transpulmonary pressure: the EPVENT 2 trial. J Thorac Dis 11(Suppl 15):S2012-S2017

450. Serpa Neto A, Cardoso SO, Manetta JA et al (2012) Association between use of lung-protective ventilation with lower tidal volumes and clinical outcomes among patients without acute respiratory distress syndrome: a meta-analysis. JAMA 308(16):1651-1659

451. Pipeling MR, Fan E (2010) Therapies for refractory hypoxemia in acute respiratory distress syndrome. JAMA 304(22):2521-2527

452. Cavalcanti AB, Suzumura ÉA, Laranjeira LN et al (2017) Effect of lung recruitment and titrated positive end-expiratory pressure (PEEP) vs low PEEP on mortality in patients with acute respiratory distress syndrome: a randomized clinical trial. JAMA 318(14):1335-1345

453. Fan E, Wilcox ME, Brower RG et al (2008) Recruitment maneuvers for acute lung injury a systematic review. Am J Respir Crit Care Med 178(11):1156-1163

454. Munshi L, Del Sorbo L, Adhikari NKJ et al (2017) Prone position for acute respiratory distress syndrome. A systematic review and meta-analysis. Ann Am Thorac Soc 14(Supplement_4):S280-s8

455. Sud S, Friedrich JO, Taccone $P$ et al (2010) Prone ventilation reduces mortality in patients with acute respiratory failure and severe hypoxemia: systematic review and meta-analysis. Intensive Care Med 36(4):585-599

456. Guérin C, Reignier J, Richard JC et al (2013) Prone positioning in severe acute respiratory distress syndrome. N Engl J Med 368(23):2159-2168 
457. Jolliet P, Bulpa P, Chevrolet JC (1998) Effects of the prone position on gas exchange and hemodynamics in severe acute respiratory distress syndrome. Crit Care Med 26(12):1977-1985

458. Lamm WJ, Graham MM, Albert RK (1994) Mechanism by which the prone position improves oxygenation in acute lung injury. Am J Respir Crit Care Med 150(1):184-193

459. Stocker R, NeffT, Stein S et al (1997) Prone postioning and low-volume pressure-limited ventilation improve survival in patients with severe ARDS. Chest 111(4):1008-1017

460. Gattinoni L, Tognoni G, Pesenti A et al (2001) Effect of prone positioning on the survival of patients with acute respiratory failure. N Engl J Med 345(8):568-573

461. Guerin C, Gaillard S, Lemasson S et al (2004) Effects of systematic prone positioning in hypoxemic acute respiratory failure: a randomized controlled trial. JAMA 292(19):2379-2387

462. Klessig HT, Geiger HJ, Murray MJ et al (1992) A National Survey on the practice patterns of anesthesiologist intensivists in the use of musclerelaxants. Crit Care Med 20(9):1341-1345

463. Murray MJ, Cowen J, DeBlock H et al (2002) Clinical practice guidelines for sustained neuromuscular blockade in the adult critically ill patient. Crit Care Med 30(1):142-156

464. Hansenflaschen JH, Brazinsky S, Basile C et al (1991) USE OF sedating drugs and neuromuscular blocking-agents in patients requiring mechanical ventilation for respiratory-failure—a National Survey. JAMA 266(20):2870-2875

465. Forel JM, Roch A, Marin V et al (2006) Neuromuscular blocking agents decrease inflammatory response in patients presenting with acute respiratory distress syndrome. Crit Care Med 34(11):2749-2757

466. Gainnier M, Roch A, Forel JM et al (2004) Effect of neuromuscular blocking agents on gas exchange in patients presenting with acute respiratory distress syndrome. Crit Care Med 32(1):113-119

467. Papazian L, Forel JM, Gacouin A et al (2010) Neuromuscular blockers in early acute respiratory distress syndrome. N Engl J Med 363(12):1107-1116

468. Alhazzani W, Alshahrani M, Jaeschke R et al (2013) Neuromuscular blocking agents in acute respiratory distress syndrome: a systematic review and meta-analysis of randomized controlled trials. Crit Care 17(2):R43

469. Guervilly C, Bisbal M, Forel JM et al (2017) Effects of neuromuscular blockers on transpulmonary pressures in moderate to severe acute respiratory distress syndrome. Intensive Care Med 43(3):408-418

470. Lyu G, Wang X, Jiang W et al (2014) Clinical study of early use of neuromuscular blocking agents in patients with severe sepsis and acute respiratory distress syndrome. Zhonghua Wei Zhong Bing Ji Jiu Yi Xue 26(5):325-329

471. National Heart L, Blood Institute PCTN, Moss M et al (2019) Early Neuromuscular Blockade in the Acute Respiratory Distress Syndrome. N Engl J Med 380(21):1997-2008

472. Alhazzani W, Belley-Cote E, Moller MH, et al (2020) Neuromuscular blockade in patients with ARDS: a rapid practice guideline. Intensive Care Med

473. Tarazan N, Alshehri M, Sharif S et al (2020) Neuromuscular blocking agents in acute respiratory distress syndrome: updated systematic review and meta-analysis of randomized trials. Intensive Care Med Exp 8(1):61

474. Johnson KL, Cheung RB, Johnson SB et al (1999) Therapeutic paralysis of critically ill trauma patients: perceptions of patients and their family members. Am J Crit Care 8(1):490-498

475. Munshi L, Walkey A, Goligher E et al (2019) Venovenous extracorporeal membrane oxygenation for acute respiratory distress syndrome: a systematic review and meta-analysis. Lancet Respir Med 7(2):163-172

476. Combes A, Hajage D, Capellier G et al (2018) Extracorporeal membrane oxygenation for severe acute respiratory distress syndrome. N Engl J Med 378(21):1965-1975

477. Peek GJ, Mugford M, Tiruvoipati R et al (2009) Efficacy and economic assessment of conventional ventilatory support versus extracorporeal membrane oxygenation for severe adult respiratory failure (CESAR): a multicentre randomised controlled trial. Lancet 374(9698):1351-1363

478. Annane D, Renault A, Brun-Buisson C et al (2018) Hydrocortisone plus Fludrocortisone for Adults with Septic Shock. N Engl J Med 378(9):809-818
479. Venkatesh B, Finfer S, Cohen J et al (2018) Adjunctive glucocorticoid therapy in patients with septic shock. N Engl J Med 378(9):797-808

480. Rygård SL, Butler E, Granholm A et al (2018) Low-dose corticosteroids for adult patients with septic shock: a systematic review with meta-analysis and trial sequential analysis. Intensive Care Med 44(7):1003-1016

481. Dellinger RP, Bagshaw SM, Antonelli M et al (2018) Effect of targeted polymyxin B hemoperfusion on 28-day mortality in patients with septic shock and elevated endotoxin level: the EUPHRATES Randomized Clinical Trial. JAMA 320(14):1455-1463

482. Zhou F, Peng Z, Murugan R et al (2013) Blood purification and mortality in sepsis: a meta-analysis of randomized trials. Crit Care Med 41(9):2209-2220

483. David S, Bode C, Putensen C et al (2021) Adjuvant therapeutic plasma exchange in septic shock. Intensive Care Med 47(3):352-354

484. Hébert PC, Wells G, Blajchman MA et al (1999) a multicenter, randomized, controlled clinical trial of transfusion requirements in critical care. N Engl J Med 340(6):409-417

485. Holst LB, Haase N, Wetterslev J et al (2014) Lower versus higher hemoglobin threshold for transfusion in septic shock. N Engl J Med 371(15):1381-1391

486. Hirano Y, Miyoshi Y, Kondo Y et al (2019) Liberal versus restrictive red blood cell transfusion strategy in sepsis or septic shock: a systematic review and meta-analysis of randomized trials. Crit Care 23(1):262

487. Bergamin FS, Almeida JP, Landoni G et al (2017) Liberal versus restrictive transfusion strategy in critically ill oncologic patients: the transfusion requirements in critically ill oncologic patients randomized controlled trial. Crit Care Med 45(5):766-773

488. Hotchkiss RS, Monneret G, Payen D (2013) Immunosuppression in sepsis: a novel understanding of the disorder and a new therapeutic approach. Lancet Infect Dis 13(3):260-268

489. Madsen MB, Hjortrup PB, Hansen MB et al (2017) Immunoglobulin G for patients with necrotising soft tissue infection (INSTINCT): a randomised blinded, placebo-controlled trial. Intensive Care Med 43(11):1585-1593

490. Welte T, Dellinger RP, Ebelt $\mathrm{H}$ et al (2018) Efficacy and safety of trimodulin, a novel polyclonal antibody preparation, in patients with severe community-acquired pneumonia: a randomized, placebo-controlled, double-blind, multicenter, phase II trial (CIGMA study). Intensive Care Med 44(4):438-448

491. Alejandria MM, Lansang MA, Dans LF et al (2013) Intravenous immunoglobulin for treating sepsis, severe sepsis and septic shock. Cochrane Database Syst Rev 9:Cd001090

492. Busani S, Damiani E, Cavazzuti I et al (2016) Intravenous immunoglobulin in septic shock: review of the mechanisms of action and metaanalysis of the clinical effectiveness. Minerva Anestesiol 82(5):559-572

493. Cook DJ, Fuller HD, Guyatt GH et al (1994) Risk factors for gastrointestinal bleeding in critically ill patients. Canadian Critical Care Trials Group. N Engl J Med 330(6):377-381

494. Krag M, Marker S, Perner A et al (2018) Pantoprazole in patients at risk for gastrointestinal bleeding in the ICU. N Engl J Med 379(23):2199-2208

495. D'Silva KM, Mehta R, Mitchell M, et al (2021) Proton pump inhibitor use and risk for recurrent Clostridioides difficile infection: a systematic review and meta-analysis. Clin Microbiol Infect

496. Granholm A, Zeng L, Dionne JC et al (2019) Predictors of gastrointestinal bleeding in adult ICU patients: a systematic review and meta-analysis. Intensive Care Med 45(10):1347-1359

497. Cook D, Crowther M, Meade M et al (2005) Deep venous thrombosis in medical-surgical critically ill patients: prevalence, incidence, and risk factors. Crit Care Med 33(7):1565-1571

498. Alhazzani W, Lim W, Jaeschke RZ et al (2013) Heparin thromboprophylaxis in medical-surgical critically ill patients: a systematic review and meta-analysis of randomized trials. Crit Care Med 41(9):2088-2098

499. Kahn SR, Lim W, Dunn AS et al (2012) Prevention of VTE in nonsurgical patients: antithrombotic therapy and prevention of thrombosis, 9th ed: American College of Chest Physicians Evidence-Based Clinical Practice Guidelines. Chest 141 (2 Suppl):e195S-e226S

500. Arabi YM, Al-Hameed F, Burns KEA et al (2019) Adjunctive intermittent pneumatic compression for venous thromboprophylaxis. N Engl J Med 380(14):1305-1315 
501. Kellum JA, Angus DC, Johnson JP et al (2002) Continuous versus intermittent renal replacement therapy: a meta-analysis. Intensive Care Med 28(1):29-37

502. Tonelli M, Manns B, Feller-Kopman D (2002) Acute renal failure in the intensive care unit: a systematic review of the impact of dialytic modality on mortality and renal recovery. Am J Kidney Dis 40(5):875-885

503. Zha J, Li C, Cheng G et al (2019) The efficacy of renal replacement therapy strategies for septic-acute kidney injury: a PRISMA-compliant network meta-analysis. Medicine (Baltimore) 98(16):e15257

504. Zhao Y, Chen Y (2020) Effect of renal replacement therapy modalities on renal recovery and mortality for acute kidney injury: A PRISMA-compliant systematic review and meta-analysis. Semin Dial 33(2):127-132

505. Zarbock A, Kellum JA, Schmidt C et al (2016) Effect of early vs delayed initiation of renal replacement therapy on mortality in critically ill patients with acute kidney injury: the ELAIN Randomized Clinical Trial. JAMA 315(20):2190-2199

506. Gaudry S, Hajage D, Schortgen F et al (2016) Initiation strategies for renal-replacement therapy in the intensive care unit. N Engl J Med 375(2):122-133

507. Barbar SD, Clere-Jehl R, Bourredjem A et al (2018) Timing of renalreplacement therapy in patients with acute kidney injury and sepsis. $\mathrm{N}$ Engl J Med 379(15):1431-1442

508. Investigators S-A, Canadian Critical Care Trials G, Australian et al (2020) Timing of Initiation of Renal-Replacement Therapy in Acute Kidney Injury. N Engl J Med 383(3):240-251

509. Badawi O, Waite MD, Fuhrman SA et al (2012) Association between intensive care unit-acquired dysglycemia and in-hospital mortality. Crit Care Med 40(12):3180-3188

510. Krinsley JS (2008) Glycemic variability: a strong independent predicto of mortality in critically ill patients. Crit Care Med 36(11):3008-3013

511. Siegelaar SE, Hermanides J, Oudemans-van Straaten HM et al (2010) Mean glucose during ICU admission is related to mortality by a $U$-shaped curve in surgical and medical patients: a retrospective cohort study. Crit Care 14(6):R224

512. Diabetes Care in the Hospital (2018) Standards of Medical Care in Diabetes - 2018. Diabetes Care 41(Supplement 1):S144-S151

513. van den Berghe $G$, Wouters $P$, Weekers F et al (2001) Intensive insulin therapy in critically ill patients. N Engl J Med 345(19):1359-1367

514. Brunkhorst FM, Engel C, Bloos F et al (2008) Intensive insulin therapy and pentastarch resuscitation in severe sepsis. N Engl J Med 358(2):125-139

515. Preiser JC, Devos P, Ruiz-Santana S et al (2009) A prospective randomised multi-centre controlled trial on tight glucose control by intensive insulin therapy in adult intensive care units: the Glucontrol study. Intensive Care Med 35(10):1738-1748

516. Griesdale DE, de Souza RJ, van Dam RM et al (2009) Intensive insulin therapy and mortality among critically ill patients: a meta-analysis including NICE-SUGAR study data. CMAJ 180(8):821-827

517. Song $F$, Zhong $L$, Han $L$, et al (2014) Intensive insulin therapy for septic patients: a meta-analysis of randomized controlled trials. Biomed Res Int 2014:698265. https://doi.org/10.1155/2014/698265

518. The NICE-SUGAR Study Investigators. Intensive versus conventional glucose control in critically ill patients. N Engl J Med. 2009;360(13):1283-1297

519. Yatabe $T$, Inoue $S$, Sakaguchi M et al (2017) The optimal target for acute glycemic control in critically ill patients: a network meta-analysis. Intensive Care Med 43(1):16-28

520. Kuhn SO, Meissner K, Mayes LM et al (2018) Vitamin C in sepsis. Curr Opin Anaesthesiol 31(1):55-60

521. Marik PE, Khangoora V, Rivera R et al (2017) Hydrocortisone, vitamin $C$, and thiamine for the treatment of severe sepsis and septic shock: a retrospective before-after study. Chest 151(6):1229-1238

522. Putzu A, Daems AM, Lopez-Delgado JC et al (2019) The effect of vitamin c on clinical outcome in critically ill patients: a systematic review with meta-analysis of randomized controlled trials. Crit Care Med 47(6):774-783

523. Fowler AA 3rd, Truwit JD, Hite RD et al (2019) Effect of vitamin C infusion on organ failure and biomarkers of inflammation and vascular injury in patients with sepsis and severe acute respiratory failure: the CITRIS-ALI Randomized Clinical Trial. JAMA 322(13):1261-1270
524. Fujii T, Luethi N, Young PJ et al (2020) Effect of vitamin C, hydrocortisone, and thiamine vs hydrocortisone alone on time alive and free of vasopressor support among patients with septic shock: the VITAMINS Randomized Clinical Trial. JAMA 323(5):423-431

525. Moskowitz A, Huang DT, Hou PC et al (2020) Effect of ascorbic acid, corticosteroids, and thiamine on organ injury in septic shock: the ACTS Randomized Clinical Trial. JAMA 324(7):642-650

526. Cooper DJ, Walley KR, Wiggs BR et al (1990) Bicarbonate does not improve hemodynamics in critically ill patients who have lactic acidosis. A prospective, controlled clinical study. Ann Intern Med 112(7):492-498

527. Mathieu D, Neviere R, Billard V et al (1991) Effects of bicarbonate therapy on hemodynamics and tissue oxygenation in patients with lactic acidosis: a prospective, controlled clinical study. Crit Care Med 19(11):1352-1356

528. Jaber S, Paugam C, Futier E et al (2018) Sodium bicarbonate therapy for patients with severe metabolic acidaemia in the intensive care unit (BICAR-ICU): a multicentre, open-label, randomised controlled, phase 3 trial. Lancet 392(10141):31-40

529. Kudsk KA (2002) Current aspects of mucosal immunology and its influence by nutrition. Am J Surg 183(4):390-398

530. McClave SA, Heyland DK (2009) The physiologic response and associated clinical benefits from provision of early enteral nutrition. Nutr Clin Pract 24(3):305-315

531. Reignier J, Boisramé-Helms J, Brisard L et al (2018) Enteral versus parenteral early nutrition in ventilated adults with shock: a randomised, controlled, multicentre, open-label, parallel-group study (NUTRIREA-2). Lancet 391(10116):133-143

532. Ibrahim EH, Mehringer L, Prentice D et al (2002) Early versus late enteral feeding of mechanically ventilated patients: results of a clinical trial. JPEN J Parenter Enteral Nutr 26(3):174-181

533. Malhotra A, Mathur AK, Gupta S (2004) Early enteral nutrition after surgical treatment of gut perforations: a prospective randomised study. J Postgrad Med 50(2):102-106

534. Pupelis G, Austrums E, Jansone A et al (2000) Randomised trial of safety and efficacy of postoperative enteral feeding in patients with severe pancreatitis: preliminary report. Eur J Surg 166(5):383-387

535. Singh G, Ram RP, Khanna SK (1998) Early postoperative enteral feeding in patients with nontraumatic intestinal perforation and peritonitis. J Am Coll Surg 187(2):142-146

536. Ely EW (2017) The ABCDEF Bundle: science and philosophy of how ICU liberation serves patients and families. Crit Care Med 45(2):321-330

537. Brinkman-Stoppelenburg A, Rietjens JA, van der Heide A (2014) The effects of advance care planning on end-of-life care: a systematic review. Palliat Med 28(8):1000-1025

538. White DB, Angus DC, Shields AM et al (2018) A randomized trial of a family-support intervention in intensive care units. N Engl J Med 378(25):2365-2375

539. Schneiderman $\sqcup$, Gilmer T, Teetzel HD (2000) Impact of ethics consultations in the intensive care setting: a randomized, controlled trial. Crit Care Med 28(12):3920-3924

540. Schneiderman $\sqcup$, Gilmer T, Teetzel HD et al (2003) Effect of ethics consultations on nonbeneficial life-sustaining treatments in the intensive care setting: a randomized controlled trial. JAMA 290(9):1166-1172

541. Chen C, Michaels J, Meeker MA (2019) Family outcomes and perceptions of end-of-life care in the intensive care unit: a mixed-methods review. J Palliat Care 35(3):143-153. https://doi.org/10.1177/08258 59719874767

542. Andereck WS, McGaughey JW, Schneiderman L et al (2014) Seeking to reduce nonbeneficial treatment in the ICU: an exploratory trial of proactive ethics intervention. Crit Care Med 42(4):824-830

543. Carson SS, Cox CE, Wallenstein S et al (2016) Effect of palliative care-led meetings for families of patients with chronic critical illness: a randomized clinical trial. JAMA 316(1):51-62

544. Picker D, Dans M, Heard K et al (2017) A randomized trial of palliative care discussions linked to an automated early warning system alert. Crit Care Med 45(2):234-240

545. Cheung W, Aggarwal G, Fugaccia E et al (2010) Palliative care teams in the intensive care unit: a randomised, controlled, feasibility study. Crit Care Resusc 12(1):28-35 
546. Curtis JR, Nielsen EL, Treece PD et al (2011) Effect of a quality-improvement intervention on end-of-life care in the intensive care unit: a randomized trial. Am J Respir Crit Care Med 183(3):348-355

547. Lautrette A, Darmon M, Megarbane B et al (2007) A communication strategy and brochure for relatives of patients dying in the ICU. N Engl J Med 356(5):469-478

548. Ma J, Chi S, Buettner B et al (2019) Early palliative care consultation in the medical ICU: a cluster randomized crossover trial. Crit Care Med 47(12):1707-1715

549. Clark E, MacCrosain A, Ward NS et al (2020) The key features and role of peer support within group self-management interventions for stroke? A systematic review. Disabil Rehabil 42(3):307-316

550. Govindan S, Iwashyna TJ, Watson SR et al (2014) Issues of survivorship are rarely addressed during intensive care unit stays. Baseline results from a statewide quality improvement collaborative. Ann Am Thorac Soc 11(4):587-591

551. Wobma R, Nijland RH, Ket JC et al (2016) Evidence for peer support in rehabilitation for individuals with acquired brain injury: a systematic review. J Rehabil Med 48(10):837-840

552. McPeake J, Hirshberg EL, Christie LM et al (2019) Models of peer support to remediate post-intensive care xyndrome: a report developed by the Society of Critical Care Medicine Thrive International Peer Support Collaborative. Crit Care Med 47(1):e21-e27

553. Mikkelsen ME, Jackson JC, Hopkins RO et al (2016) Peer support as a novel strategy to mitigate post-intensive care syndrome. AACN Adv Crit Care 27(2):221-229

554. Halm MA (1990) Effects of support groups on anxiety of family members during critical illness. Heart Lung 19(1):62-71

555. Fridlund B, Stener-Bengtsson A, Wannman AL (1993) Social support and social network after acute myocardial infarction; the critically ill male patient's needs, choice and motives. Intensive Crit Care Nurs 9(2):88-94

556. McPeake J, Shaw M, Iwashyna TJ et al (2017) Intensive care syndrome: promoting independence and return to employment (InS:PIRE). Early evaluation of a complex intervention. PLoS One 12(11):e0188028

557. Sabo KA, Kraay C, Rudy E et al (1989) ICU family support group sessions: family members' perceived benefits. Appl Nurs Res 2(2):82-89

558. Parent N, Fortin F (2000) A randomized, controlled trial of vicarious experience through peer support for male first-time cardiac surgery patients: impact on anxiety, self-efficacy expectation, and self-reported activity. Heart Lung 29(6):389-400

559. Damianakis T, Tough A, Marziali E et al (2016) Therapy online: A webbased video support group for family caregivers of survivors with traumatic brain injury. J Head Trauma Rehabil 31(4):E12-20

560. Harvey C, Dixon M, Padberg N (1995) Support group for families of trauma patients: a unique approach. Crit Care Nurse 15(4):59-63

561. Jones C, Macmillan RR, Griffiths RD (1994) Providing psychological support for patients after critical illness. Clin Intensive Care 5(4):176-179

562. Peskett M, Gibb P (2009) Developing and setting up a patient and relatives intensive care support group. Nurs Crit Care 14(1):4-10

563. Sacco TL, Stapleton MF, Ingersoll GL (2009) Support groups facilitated by families of former patients: creating family-inclusive critical care units. Crit Care Nurse 29(3):36-45

564. Haines KJ, Beesley SJ, Hopkins RO et al (2018) Peer support in critical care: a systematic review. Crit Care Med 46(9):1522-1531

565. Danesh V (2019) A prospective, 2-arm, single-blind, randomized controlled clinical feasibility trial design is planned. Forty $\mathrm{CCl}$ survivors will be randomized (1:1) to either the PS-PICS (peer support) intervention or usual care (control) group. NCT03788096 2019 Available from: https:/ clinicaltrials.gov/ct2/show/study/NCT03788096

566. Haines KJHC, Cranwell K, Skinner EH, Holton S, MacLeod-Smith B, Bates S, Iwashyna TJ, French C, Booth S, Carmody J (2019) Development of a peer support model using experience-based co-design to improve critical care recovery. Crit Care Explor. 1(3):e0006

567. Matthaeus-Kraemer CT, Thomas-Rueddel DO, Schwarzkopf D et al (2016) Crossing the handover chasm: Clinicians' perceptions of barriers to the early detection and timely management of severe sepsis and septic shock. J Crit Care 36:85-91

568. Parent B, LaGrone LN, Albirair MT et al (2018) Effect of standardized handoff curriculum on improved clinician preparedness in the intensive care unit: a stepped-wedge cluster randomized clinical trial. JAMA Surg 153(5):464-470
569. Nanchal R, Aebly B, Graves G et al (2017) Controlled trial to improve resident sign-out in a medical intensive care unit. BMJ Qual Saf 26(12):987-992

570. Hess DR, Tokarczyk A, O'Malley M et al (2010) The value of adding a verbal report to written handoffs on early readmission following prolonged respiratory failure. Chest 138(6):1475-1479

571. Hoffman RL, Saucier J, Dasani S et al (2017) Development and implementation of a risk identification tool to facilitate critical care transitions for high-risk surgical patients. Int J Qual Health Care 29(3):412-419

572. Chaboyer W, Lin F, Foster M et al (2012) Redesigning the ICU nursing discharge process: a quality improvement study. Worldviews Evid Based Nurs 9(1):40-48

573. Medlock S, Eslami S, Askari M et al (2011) Improved communication in post-ICU care by improving writing of ICU discharge letters: a longitudinal before-after study. BMJ Qual Saf 20(11):967-973

574. Griffiths J, Hatch RA, Bishop J et al (2013) An exploration of social and economic outcome and associated health-related quality of life after critical illness in general intensive care unit survivors: a 12-month follow-up study. Crit Care 17(3):R100

575. Donnelly JP, Lakkur S, Judd SE et al (2018) Association of neighborhood socioeconomic status with risk of infection and sepsis. Clin Infect Dis 66(12):1940-1947

576. Koch K, Norgaard M, Schonheyder HC et al (2013) Effect of socioeconomic status on mortality after bacteremia in working-age patients $A$ Danish population-based cohort study. PLoS One 8(7):e70082

577. Ho KM, Dobb GJ, Knuiman M et al (2008) The effect of socioeconomic status on outcomes for seriously ill patients: a linked data cohort study. Med J Aust 189(1):26-30

578. Ogundipe F, Kodadhala V, Ogundipe T et al (2019) Disparities in sepsis mortality by region, urbanization, and race in the USA: a Multiple Cause of Death Analysis. J Racial Ethn Health Dispar 6(3):546-551

579. Goodwin AJ, Nadig NR, MCElligott JT et al (2016) Where you live matters: the impact of place of residence on severe sepsis incidence and mortality. Chest 150(4):829-836

580. Prescott HC, Angus DC (2018) Enhancing recovery from sepsis: a review. JAMA 319(1):62-75

581. Gruther W, Pieber K, Steiner I et al (2017) Can early rehabilitation on the general ward after an intensive care unit stay reduce hospital length of stay in survivors of critical illness?: A randomized controlled trial. Am J Phys Med Rehabil 96(9):607-615

582. Huang CY, Daniels R, Lembo A et al (2019) Life after sepsis: an international survey of survivors to understand the post-sepsis syndrome. Int J Qual Health Care 31(3):191-198

583. Azoulay E, Pochard F, Chevret $\mathrm{S}$ et al (2002) Impact of a family information leaflet on effectiveness of information provided to family members of intensive care unit patients: a multicenter, prospective, randomized, controlled trial. Am J Respir Crit Care Med 165(4):438-442

584. Bench S, Day T, Heelas K et al (2015) Evaluating the feasibility and effectiveness of a critical care discharge information pack for patients and their families: a pilot cluster randomised controlled trial. BMJ Open 5(11):e006852

585. Demircelik MB, Cakmak M, Nazli Y et al (2016) Effects of multimedia nursing education on disease-related depression and anxiety in patients staying in a coronary intensive care unit. Appl Nurs Res 29:5-8

586. Fleischer S, Berg A, Behrens J et al (2014) Does an additional structured information program during the intensive care unit stay reduce anxiety in ICU patients?: a multicenter randomized controlled trial. BMC Anesthesiol 14:48

587. Gehrke-Beck S, Bänfer M, Schilling N et al (2017) The specific needs of patients following sepsis: a nested qualitative interview study. BJGP Open. 1(1):bjgpopen 17X100725

588. Schmidt K, Worrack S, Von Korff M et al (2016) Effect of a primary care management intervention on mental health-related quality of life among survivors of sepsis: a randomized clinical trial. JAMA 315(24):2703-2711

589. Oermann MH, McInerney SM (2007) An evaluation of sepsis Web sites for patient and family education. Plast Surg Nurs 27(4):192-196

590. Légaré F, Adekpedjou R, Stacey D et al (2018) Interventions for increasing the use of shared decision making by healthcare professionals. Cochrane Database Syst Rev 7(7):Cd006732 
591. Anderson WG, Arnold RM, Angus DC et al (2009) Passive decisionmaking preference is associated with anxiety and depression in relatives of patients in the intensive care unit. J Crit Care 24(2):249-254

592. Bokinskie JC (1992) Family conferences: a method to diminish transfer anxiety. J Neurosci Nurs 24(3):129-133

593. Choi J, Lingler JH, Donahoe MP et al (2018) Home discharge following critical illness: a qualitative analysis of family caregiver experience. Heart Lung 47(4):401-407

594. Moss KO, Douglas SL, Baum E et al (2019) Family surrogate decisionmaking in chronic critical IIIness: a qualitative analysis. Crit Care Nurse 39(3):e18-e26

595. Austin CA, Mohottige D, Sudore RL et al (2015) Tools to promote shared decision making in serious illness: a systematic review. JAMA Intern Med 175(7):1213-1221

596. Bell CM, Brener SS, Gunraj N et al (2011) Association of ICU or hospital admission with unintentional discontinuation of medications for chronic diseases. JAMA 306:840-847

597. Fabes J, Seligman W, Barrett C et al (2017) Does the implementation of a novel intensive care discharge risk score and nurse-led inpatient review tool improve outcome? A prospective cohort study in two intensive care units in the UK. BMJ Open 7(12):e018322

598. Mekonnen AB, McLachlan AJ, Brien JA (2016) Pharmacy-led medication reconciliation programmes at hospital transitions: a systematic review and meta-analysis. J Clin Pharm Ther 41(2):128-144

599. Morandi A, Vasilevskis E, Pandharipande PP et al (2013) Inappropriate medication prescriptions in elderly adults surviving an intensive care unit hospitalization. J Am Geriatr Soc 61:1128-1134

600. Scales DC, Fischer HD, Li P et al (2016) Unintentional continuation of medications intended for acute illness after hospital discharge: A population-based cohort study. J Gen Intern Med 31:196-202

601. Stelfox HT, Bastos J, Niven DJ et al (2016) Critical care transition programs and the risk of readmission or death after discharge from ICU. Intensive Care Med 42(3):401-410

602. Tomichek JE, Stollings JL, Pandharipande PP et al (2016) Antipsychotic prescribing patterns during and after critical illness: a prospective cohort study. Crit Care 20:378

603. Ball C, Kirkby M, Williams S (2003) Effect of the critical care outreach team on patient survival to discharge from hospital and readmission to critical care: non-randomised population based study. BMJ 327(7422):1014

604. Baxter AD, Cardinal P, Hooper J et al (2008) Medical emergency teams at The Ottawa Hospital: the first two years. Can J Anaesth 55(4):223-231

605. Choi S, Lee J, Shin Y et al (2016) Effects of a medical emergency team follow-up programme on patients discharged from the medical intensive care unit to the general ward: a single-centre experience. J Eval Clin Pract 22(3):356-362

606. Elliott D, McKinley S, Alison J et al (2011) Health-related quality of life and physical recovery after a critical illness: a multi-centre randomised controlled trial of a home-based physical rehabilitation program. Crit Care 15:R142

607. Garcea G, Thomasset S, McClelland L et al (2004) Impact of a critical care outreach team on critical care readmissions and mortality. Acta Anaesthesiol Scand 48(9):1096-1100

608. Green A, Edmonds L (2004) Bridging the gap between the intensive care unit and general wards-the ICU Liaison Nurse. Intensive Crit Care Nurs 20(3):133-143

609. Leary T, Ridley S (2003) Impact of an outreach team on re-admissions to a critical care unit. Anaesthesia 58(4):328-332

610. Pittard AJ (2003) Out of our reach? Assessing the impact of introducing a critical care outreach service. Anaesthesia 58(9):882-885

611. Williams TA, Leslie G, Finn J et al (2010) Clinical effectiveness of a critical care nursing outreach service in facilitating discharge from the intensive care unit. Am J Crit Care 19(5):e63-72

612. Pronovost P, Weast B, Schwarz M et al (2003) Medication reconciliation: a practical tool to reduce the risk of medication errors. J Crit Care 18(4):201-205

613. Ravn-Nielsen LV, Duckert ML, Lund ML et al (2018) Effect of an in-hospital multifaceted clinical pharmacist intervention on the risk of readmission: a randomized clinical trial. JAMA Intern Med 178(3):375-382
614. Taylor SP, Chou SH, Sierra MF et al (2020) Association between Adherence to Recommended Care and Outcomes for Adult Survivors of Sepsis. Ann Am Thorac Soc 17(1):89-97

615. Etesse B, Jaber S, Mura T et al (2010) How the relationships between general practitioners and intensivists can be improved: the general practitioners' point of view. Crit Care 14(3):R112

616. Kripalani S, LeFevre F, Phillips CO et al (2007) Deficits in communication and information transfer between hospital-based and primary care physicians: implications for patient safety and continuity of care. JAMA 297:831-841

617. Robelia PM, Kashiwagi DT, Jenkins SM et al (2017) Information transfer and the hospital discharge summary: National primary care provider perspectives of challenges and opportunities. J Am Board Fam Med 30(6):758-765

618. Weissman GE, Harhay MO, Lugo RM et al (2016) Natural language processing to assess documentation of features of critical illness in discharge documents of acute respiratory distress syndrome survivors. Ann Am Thorac Soc 13(9):1538-1545

619. Needham DM, Davidson J, Cohen H et al (2012) Improving long-term outcomes after discharge from intensive care unit: report from a stakeholders' conference. Crit Care Med 40:502-509

620. Iwashyna TJ, Ely EW, Smith DM et al (2010) Long-term cognitive impairment and functional disability among survivors of severe sepsis. JAMA 304:1787-1794

621. Konig C, Matt B, Kortgen A et al (2019) What matters most to sepsis survivors: a qualitative analysis to identify specific health-related quality of life domains. Qual Life Res 28(3):637-647

622. Dietz BW, Jones TK, Small DS et al (2017) The relationship between index hospitalizations, sepsis, and death or transition to hospice care during 30-day hospital readmissions. Med Care 55(4):362-370

623. Ortego A, Gaieski DF, Fuchs BD et al (2015) Hospital-based acute care use in survivors of septic shock. Crit Care Med 43(4):729-737

624. Mayr FB, Talisa VB, Balakumar V et al (2017) Proportion and cost of unplanned 30-day readmissions after sepsis compared with other medical conditions. JAMA 317(5):530-531

625. Hernandez AF, Greiner MA, Fonarow GC et al (2010) Relationship between early physician follow-up and 30-day readmission among Medicare beneficiaries hospitalized for heart failure. JAMA 303(17):1716-1722

626. Field TS, Ogarek J, Garber L et al (2015) Association of early post-discharge follow-up by a primary care physician and 30-day rehospitalization among older adults. J Gen Intern Med 30(5):565-571

627. Shen E, Koyama SY, Huynh DN et al (2017) Association of a dedicated post-hospital discharge follow-up visit and 30-Day readmission risk in a Medicare Advantage population. JAMA Intern Med 177(1):132-135

628. Douglas SL, Daly BJ, Kelley CG et al (2007) Chronically critically ill patients: health-related quality of life and resource use after a disease management intervention. Am J Crit Care 16(5):447-457

629. Jónasdóttir RJ, Jónsdóttir H, Gudmundsdottir B et al (2018) Psychological recovery after intensive care: Outcomes of a long-term quasi-experimental study of structured nurse-led follow-up. Intensive Crit Care Nurs 44:59-66

630. Kansagara D, Ramsay RS, Labby D et al (2012) Post-discharge intervention in vulnerable, chronically ill patients. J Hosp Med 7(2):124-130

631. Deb P, Murtaugh CM, Bowles KH et al (2019) Does early follow-up improve the outcomes of sepsis survivors discharged to home health care? Med Care 57(8):633-640

632. Annane D, Sharshar T (2015) Cognitive decline after sepsis. Lancet Respir Med 3(1):61-69

633. Jackson JC, Ely EW, Morey MC et al (2012) Cognitive and physical rehabilitation of intensive care unit survivors: results of the RETURN randomized controlled pilot investigation. Crit Care Med 40(4):1088-1097

634. Brummel NE, Girard TD, Ely EW et al (2014) Feasibility and safety of early combined cognitive and physical therapy for critically ill medical and surgical patients: the Activity and Cognitive Therapy in ICU (ACT-ICU) trial. Intensive Care Med 40(3):370-379

635. Zhao J, Yao L, Li M et al (2019) Effects of early intervention training on cognitive impairment in critical patients. Zhonghua Wei Zhong Bing Ji Jiu Yi Xue 31(3):298-302 
636. Wong GKC, Mak JSY, Wong A et al (2017) Minimum clinically important difference of Montreal Cognitive Assessment in aneurysmal subarachnoid hemorrhage patients. J Clin Neurosci 46:41-44

637. Teixeira C, Rosa RG (2018) Post-intensive care outpatient clinic: is it feasible and effective? A literature review. Rev Bras Ter Intensiva 30(1):98-111

638. Cuthbertson BH, Rattray J, Campbell MK et al (2009) The PRaCTICaL study of nurse led, intensive care follow-up programmes for improving long term outcomes from critical illness: a pragmatic randomised controlled trial. BMJ 339:b3723

639. Jensen JF, Egerod I, Bestle MH et al (2016) A recovery program to improve quality of life, sense of coherence and psychological health in ICU survivors: a multicenter randomized controlled trial, the RAPIT study. Intensive Care Med 42(11):1733-1743

640. Schofield-Robinson OJ, Lewis SR, Smith AF et al (2018) Follow-up services for improving long-term outcomes in intensive care unit (ICU) survivors. Cochrane Database Syst Rev 11:CD012701

641. Kowalkowski M, Chou SH, McWilliams A et al (2019) Structured, proactive care coordination versus usual care for Improving Morbidity during Post-Acute Care Transitions for Sepsis (IMPACTS): a pragmatic, randomized controlled trial. Trials 20(1):660

642. Paratz JD, Kenardy J, Mitchell G et al (2014) IMPOSE (IMProving Outcomes after Sepsis)-the effect of a multidisciplinary follow-up service on health-related quality of life in patients postsepsis syndromes-a double-blinded randomised controlled trial: protocol. BMJ Open 4(5):e004966

643. Prescott HC, Iwashyna TJ, Blackwood B et al (2019) Understanding and enhancing sepsis survivorship. Priorities for research and practice. Am J Respir Crit Care Med 200(8):972-981

644. Batterham AM, Bonner S, Wright J et al (2014) Effect of supervised aerobic exercise rehabilitation on physical fitness and quality-of-life in survivors of critical illness: an exploratory minimized controlled trial (PIX study). Br J Anaesth 113:130-137

645. Battle C, James K, Temblett P et al (2019) Supervised exercise rehabilitation in survivors of critical illness: a randomised controlled trial. J Intensive Care Soc 20(1):18-26

646. Connolly B, Thompson A, Douiri A et al (2015) Exercise-based rehabilitation after hospital discharge for survivors of critical illness with intensive care unit-acquired weakness: a pilot feasibility trial. J Crit Care 30(3):589-598

647. Jones C, Skirrow P, Griffiths RD et al (2003) Rehabilitation after critical illness: a randomized, controlled trial. Crit Care Med 31:2456-2461

648. Jones TK, Fuchs BD, Small DS et al (2015) Post-acute care use and hospital readmission after sepsis. Ann Am Thorac Soc 12(6):904-913

649. McDowell K, O’Neill B, Blackwood B et al (2017) Effectiveness of an exercise programme on physical function in patients discharged from hospital following critical illness: a randomised controlled trial (the REVIVE trial). Thorax 72(7):594-595

650. McWilliams DJ, Benington S, Atkinson D (2016) Outpatient-based physical rehabilitation for survivors of prolonged critical illness: a randomized controlled trial. Physiother Theory Pract 32(3):179-190

651. Walsh TS, Salisbury LG, Merriweather JL et al (2015) Increased hospitalbased physical rehabilitation and information provision after intensive care unit discharge: The RECOVER randomized clinical trial. JAMA Intern Med 175:901-910

652. Health NIf, Excellence C (2014) Rehabilitation after critical illness in adults: NICE Reino Unido; 2014. https://www.nice.org.uk/guidance/ qs158/resources/rehabilitation-after-critical-illness-in-adults-pdf-75545 546693317. Accessed 17 Mar 2021

653. Major ME, Kwakman R, Kho ME et al (2016) Surviving critical illness: what is next? An expert consensus statement on physical rehabilitation after hospital discharge. Crit Care 2016 20:354 